\title{
SINGLE-PULSE STUDY OF RADIO PULSARS
}

\author{
Anna V. Bilous \\ Chernivtsy, Ukraine
}

B.Sc., Moscow Institute of Physics and Technology, 2006

M.Sc., Moscow Institute of Physics and Technology, 2008

M.Sc., University of Virginia, 2010

A Dissertation Presented to the Graduate

Faculty of the University of Virginia

in Candidacy for the Degree of

Doctor of Philosophy

Department of Astronomy

University of Virginia

August, 2012

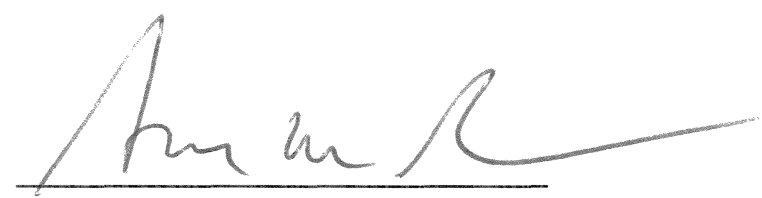

Dr. Scott M. Ransom

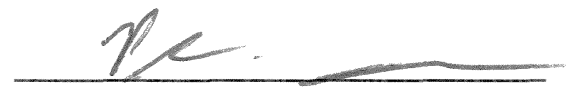

Dr. Philip L. Arras

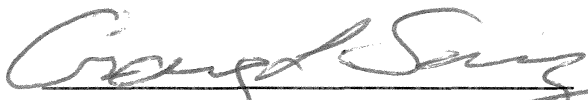

Dr. Gaig L. Sarazin

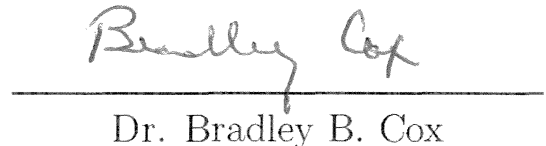

Dr. Bradley B. Cox

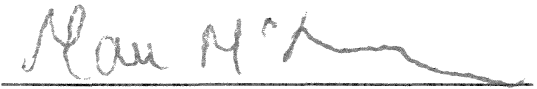

Dr. Maura A. McLaughlin 
Epigraph 


\section{Robert Sheckley, "Mindswap":}

“... but despite this, a few tentative rules might be adduced for the suicidal traveller to the Twisted World:

Remember that all rules may lie, in the Twisted World, including this rule which points out the exception, and including this modifying clause which invalidates the exception ... ad infinitum. But also remember that no rule necessarily lies; that any rule may be true, including this rule and its exceptions.

A wise man once asked, 'What would happen if I could enter the Twisted World without preconceptions?' A final answer to his question is impossible; but we would hazard that he would have some preconceptions by the time he came out. Lack of opinion is not armour. Some men feel that the height of intelligence is the discovery that all things may be reversed, and thereby become their opposites. Many clever games can be played with this proposition, but we do not advocate its use in the Twisted World. There all doctrines are equally arbitrary, including the doctrine of the arbitrariness of doctrines. Do not expect to outwit the Twisted World. It is bigger, smaller, longer and shorter than you; it does not prove; it is. Something that is never has to prove anything. All proofs are attempts at becoming. A proof is true only to itself, and it implies nothing except the existence of proofs, which prove nothing. Anything that is, is improbable, since everything is extraneous, unnecessary,and a threat to the reason. Three comments concerning the Twisted World may have nothing to do with the Twisted World. The traveller is warned." 
Abstract 
Single pulses provide valuable information about the pulsar magnetosphere, giving more spatial and time resolution than the integrated pulse profiles. Clearly, there are several different types of single pulse emission, however it is still unknown what is the nature or even strict definition of each type. This work aims to shed some light on different kinds of single pulses, making an attempt to constrain some emission theories and trace the possible connection between different types. The thesis consists of following case studies:

1. No apparent correlation was found between giant pulses (GPs) from the Crab pulsar (9 GHz, Green Bank Telescope) and its gamma-ray photons (100 MeV $5 \mathrm{GeV}$, Fermi). This result suggests that GPs, at least the ones detected at high radio frequencies, are due to changes in coherence of radio emission rather than an overall increase in the magnetospheric particle density. Also, no apparent correlation was found between Crab GPs (1.5 GHz, Green Bank Telescope) and X-ray photons $(1.5-4.5 \mathrm{keV}$, Chandra).

2. We show that single pulses from the millisecond pulsar B1821-24A fall into two distinct categories - broad faint pulses, coincident with the peaks of integrated pulse profile and bright narrow GPs on the trailing edges of profile components. Owing to our large fractional bandwidth we were able to prove the hypothesis that the spectra of these GPs consist of separate substantially polarized patches.

3. We present a LOFAR study of single pulses from pulsars B0809+74 and B1133+16 below $90 \mathrm{MHz}$. We show that the spectral width of bright, low-frequency pulses scales with increasing frequency as $\Delta f / f \sim 0.15$, at least in the case of PSR $\mathrm{B} 0809+74$. This behaviour is consistent with predictions of strong plasma turbulence model.

4. Millisecond pulsar B1744-24A belongs to "windy" binary systems, with unbound material escaping from the companion star and interacting with the pulsar's magnetosphere. B1744-24A appears to emit strong wide single pulses, which occur preferentially shortly before and after the pulsar is obscured by the 
unbound material from the companion star. We analyze the properties of these single pulses and compare them to the giant pulses. 
Acknowledgements 
This work is dedicated to all my friends, both in Charlottesville and overseas, from whom I learned a lot, sometimes in the most unexpected way.

Also I would like to thank people, whose support, criticism, ideas and overall positive attitude made this work possible. Many thanks to Vlad Kondratiev, the Ransom Factory at NRAO (Scott Ransom, Paul Demorest, Ryan Lynch, Tim Pennucci, Tuck Sanpa-arsa, Brian Prager!), the LOFAR Pulsar Working Group (Jason Hessels, Joeri van Leeuwen, Ben Stappers and many other), Maura McLaughlin, Maxim Lyutikov and David Nice. It was a big pleasure. 
Note 
Chapter 2: the work was done in collaboration with V. Kondratiev (ASTRON, the Netherlands), M. McLaughlin (West Virginia University, NRAO), S. Ransom (NRAO), M. Lyutikov (Purdue University), M. Mickaliger (West Virginia University) and G. Langston (NRAO)

Published as Bilous et al., 2011, ApJ, 728, 110

Chapter 3: the work was conducted in collaboration with M. McLaughlin, V. Kondratiev and S. Ransom

Published as Bilous et al., 2012, ApJ, 749, 24

Chapter 4: the work was conducted in collaboration with T. Pennucci (University of Virginia), P. Demorest (NRAO), S. Ransom, I. Stairs (University of British Columbia), J. Hessels (ASTRON/University of Amsterdam) and P. Freire (Max-Planck-Institut für Radioastronomie)

Will be published as Bilous et al., 201(2?)

Chapter 5: the results presented are a part of a low-frequency single-pulse survey, conducted by V. Kondratiev, J. Hessels, B. Stappers (University of Manchester) and LOFAR Pulsar Working Group.

AB significantly contributed to the search algorithm development (Section 5.2), statistical analysis (in particular for Section 5.3.1) and the interpretation of the results.

Will be published as Kondratiev et al., 201(3?)

Chapter 6: the work was conducted in collaboration with S. Ransom, D. Nice (Lafayette College ), T. Pennucci, P. Demorest, I. Stairs, J. Hessels and P. Freire

Will be published as Bilous et al., 201(3?) 


\section{Table of contents}

Epigraph

Abstract iv

Acknowledgements vii

Note ix

1 Introduction 1

1.1 General background . . . . . . . . . . . . . . . . 2

1.2 Radio emission . . . . . . . . . . . . . . . . . . . 3

2 Correlation of Fermi Photons with High-Frequency Radio Giant Pulses From The Crab Pulsar 9

2.1 Introduction . . . . . . . . . . . . . . . . . . . . . . 10

2.2 Radio observations . . . . . . . . . . . . . . . . . . . . 13

2.3 Fermi data . . . . . . . . . . . . . . . . 16

2.4 Propagation effects for Giant Pulses . . . . . . . . . . . . . . 17

2.5 Correlation analysis . . . . . . . . . . . . . . . . . . . 21

2.5.1 Is GP rate correlated with single $\gamma$-ray photons? . . . . . 21

2.5.2 Does $\gamma$-ray flux change around single GPs? . . . . . . . . 22

2.6 Conclusions . . . . . . . . . . . . . . . . . . . 25

3 Correlation of Chandra Photons with the Radio Giant Pulses from the Crab Pulsar $\quad 32$

3.1 Introduction . . . . . . . . . . . . . . . . . . 33 
3.2 Radio observations . . . . . . . . . . . . . . . . . . . 36

3.3 X-ray observations . . . . . . . . . . . . . . . . . . . . . . . . 42

3.4 Correlation Analysis . . . . . . . . . . . . . . . . . 45

3.4.1 Correlation on the timescale of radio emission window . . 48

3.4.2 Correlation on the timescale of pulsar period . . . . . . 48

3.4.3 Correlation on the timescale of GP duration . . . . . . . 49

3.5 Discussion . . . . . . . . . . . . . . . . . . . 50

4 B1821-24A (M28A): Average Profile and Single Pulses 51

4.1 Introduction . . . . . . . . . . . . . . . . . . . . 52

4.2 Observations and calibration . . . . . . . . . . . . 53

4.3 Average profile . . . . . . . . . . . . . 56

4.3 .1 Spectral indices . . . . . . . . . . . . . 56

4.3 .2 Polarization . . . . . . . . . . . . . . . 57

4.4 Single pulses . . . . . . . . . . . . . . . . . . . . . . . . . 59

4.5 Summary ......................... 63

5 LOFAR Detection of Bright Low-Frequency Narrow-Band Pulses from Pulsars B0809+74 and B1133+16 75

5.1 Introduction . . . . . . . . . . . . . . . . 76

5.2 Observations and Data Reduction . . . . . . . . . . . . 78

5.2 .1 DM tweaking . . . . . . . . . . . . . . . . 79

5.2 .2 Single-pulse selection . . . . . . . . . . . . . . 80

5.2 .3 Bandpass correction . . . . . . . . . . . . 82

5.3 Results........................ 83

5.3 .1 Pulse energy distributions . . . . . . . . . . 84

5.3 .2 Peak flux densities . . . . . . . . . . . . 85

5.3 .3 Pulse spectra . . . . . . . . . . . . . . . . 87

5.3 .4 Giant pulses? . . . . . . . . . . . . . . . . . 88

5.4 The narrow-band emission of the PSR B0809+74 . . . . . . . 90

5.4 .1 ISM scintillations? . . . . . . . . . . . . . . . . 90

5.4 .2 Ionosphere? . . . . . . . . . . . . . . . . . . . . . . . . 91

5.4.3 Strong plasma turbulence models . . . . . . . . . . . 91

5.5 Summary . . . . . . . . . . . . . . . . . . 92 
6 Terzan5A: Timing and Single Pulses 101

6.1 Introduction . . . . . . . . . . . . . . . . . . . . 102

6.2 Timing . . . . . . . . . . . . . . . . . . 103

6.3 Single pulses . . . . . . . . . . . . . . . . 106

6.4 Summary . . . . . . . . . . . . . . . . . 108 
Chapter 1

\section{Introduction}




\subsection{General background}

Pulsars are rapidly rotating, strongly magnetized neutron stars, which are formed during the supernova explosion of massive stars. If the mass of the core of a progenitor star lies approximately within $1.9-2.5 M_{\odot}$ range, the collapse of the core stops when the pressure of degenerate neutrons balances the gravity. During the collapse, the core loses about one solar mass, but retains the angular momentum and the magnetic flux through surface. The radius of the core shrinks by a factor of about $10^{5}$, changing the spin period and the surface magnetic field by $10^{10}$. The canonical neutron star has $M=1.4 M_{\odot}$, $R=10 \mathrm{~km}, B=10^{12} \mathrm{G}$ and a spin period of $P \sim 0.5 \mathrm{~s}$, although the values for $P$ and $B$ are known to spread across a couple of orders of magnitude.

A neutron star's ultra-high density gives it very high surface gravity - a typical escape velocity is about one third the speed of light. However, the surface magnetic field is so strong that it completely dominates all physical processes outside the star. To the first order of approximation, the magnetic field of a pulsar is dipolar with the axis of the dipole misaligned with the spin axis. A local electric field, induced by the rotating magnetic field, pulls the charged particles out of the surface of the neutron star. Extracted plasma moves along magnetic field lines, corotating rigidly with the star up to the light cylinder radius where the co-rotation speed approaches speed of light. Though the electromagnetic waves emitted by plasma are continuous, the emission is highly beamed and we detect the signal for only a fraction of the spin period, when the direction of beam matches our line of sight.

The structure and processes in pulsar magnetosphere are still (40 years since pulsars were discovered!) far from being understood completely, although some progress had been made. Pulsars are observed throughout the full electromagnetic spectrum, from $18 \mathrm{MHz}$ in radio to $\mathrm{TeV}$ energies. Very sketchily, the optical, X-ray and $\gamma$-ray pulses are caused by synchrotron emission, curvature emission or inverse Compton scattering in either the polar cap region or in the 
outer gaps (see Fig. 1.1). Also, we observe thermal X-ray emission from the surface of neutron stars.

There have been many models proposed for pulsar radio emission. They can be divided into three basic categories. The antenna mechanisms involve particles confined in a volume of size smaller than the emitted wavelength, radiating all in phase. Relativistic plasma emission models require some kinds of reactive plasma instability and a way to convert the energy of the resulting plasma turbulence into radio waves. Finally, there have been a number of attempts to explain radio pulses with maser (negative absorption) emission.

The observed pulsed radio emission (together with high-energy radiation, magnetic dipole radiation and a pulsar wind) takes place at the expense of rotational kinetic energy of the neutron star, making it to slow down with time. Thus, we observe not only a pulsar spin period $P$, but also the corresponding rate

of spin-down, $\dot{P}$, both of which can be measured with a very high degree of accuracy. Using $P$ and $\dot{P}$ one can estimate the pulsar's age, spin-down power, and magnetic field strength, assuming that the spin-down rate is dominated by magnetic braking.

The evolution of pulsars can be very well traced on the $P-\dot{P}$ diagram (Fig. 1.2). Pulsars start their post-supernova lives in the upper left corner of the $P-\dot{P}$ plane and gradually move to the right and down as they age and slow down. The bulk of pulsars around $P \sim 0.5$ s are called 'normal pulsars', as opposed to 'millisecond pulsars' $(P \sim 3 \mathrm{~ms})$ in the left lower corner of the diagram. Millisecond pulsars are recycled pulsars, which have been spun up by accreting mass and angular momentum from their companions to the point that they emit radio pulses despite their relatively low magnetic field strengths.

\subsection{Radio emission}

Most observed (and averaged) radio emission from a pulsar is effectively a longexposed image of time-variable plasma processes in the pulsar magnetosphere. 


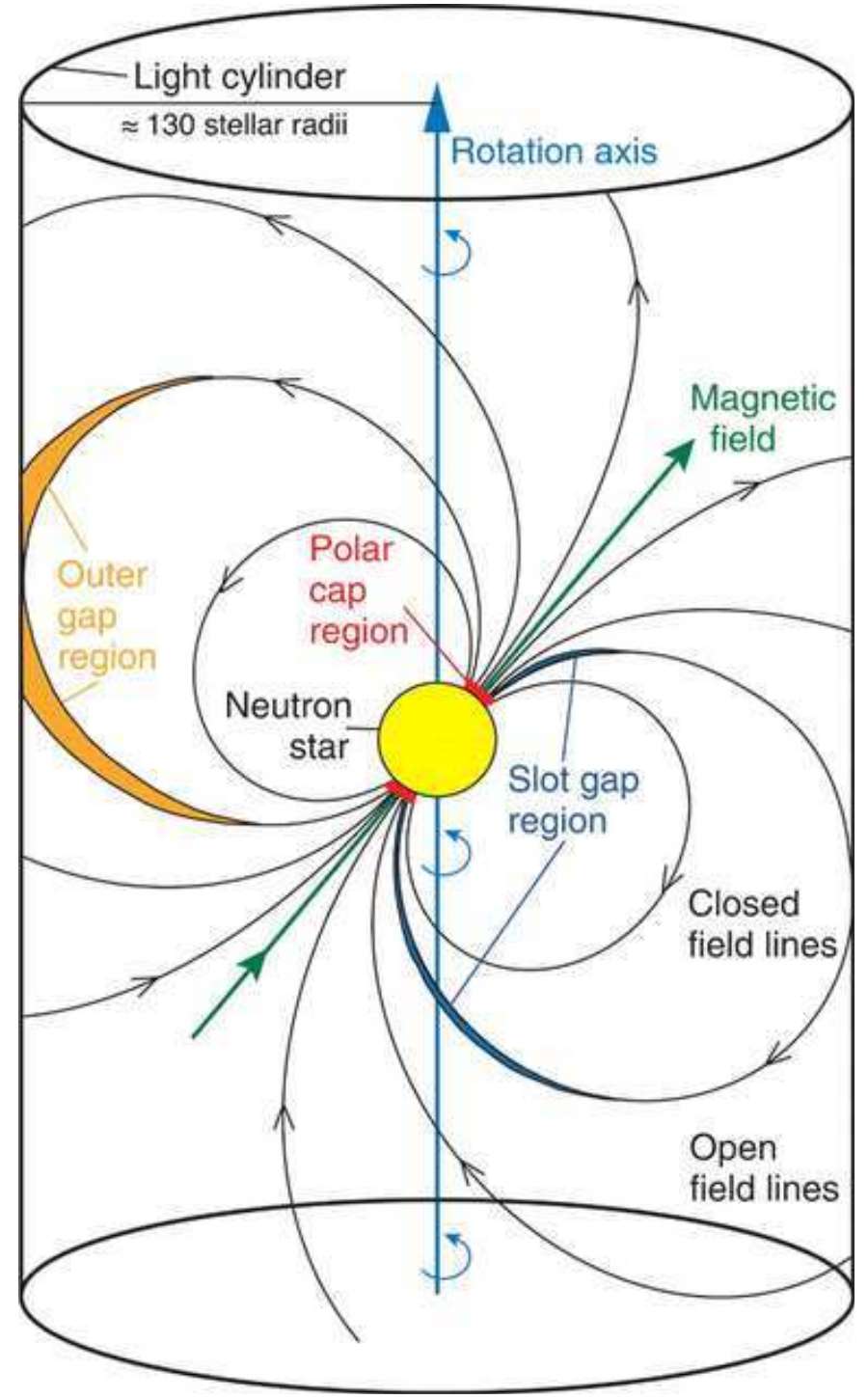

Fig. 1.1. - A common model of a rotating neutron star and its magnetosphere. Electrons are trapped and accelerated along the magnetic field lines of the pulsar and emit electromagnetic radiation via the synchrotron-curvature mechanism. Vacuum gaps or vacuum regions occur at the polar cap very close to the neutron star surface, in a thin layer extending for several stellar radii along the boundary of the closed magnetosphere, the so-called slot gap, and in the outer region close to the light cylinder (the outer gap). Vacuum gaps are filled with plasma, but its density is lower than the critical Goldreich-Julian density, in which the magnetically induced electric field is saturated, and therefore electrons in these gaps can be accelerated to very high energies. Figure and caption are from Aliu et al. (2008). The light cylinder radius is given for the pulsar in the Crab nebula. 


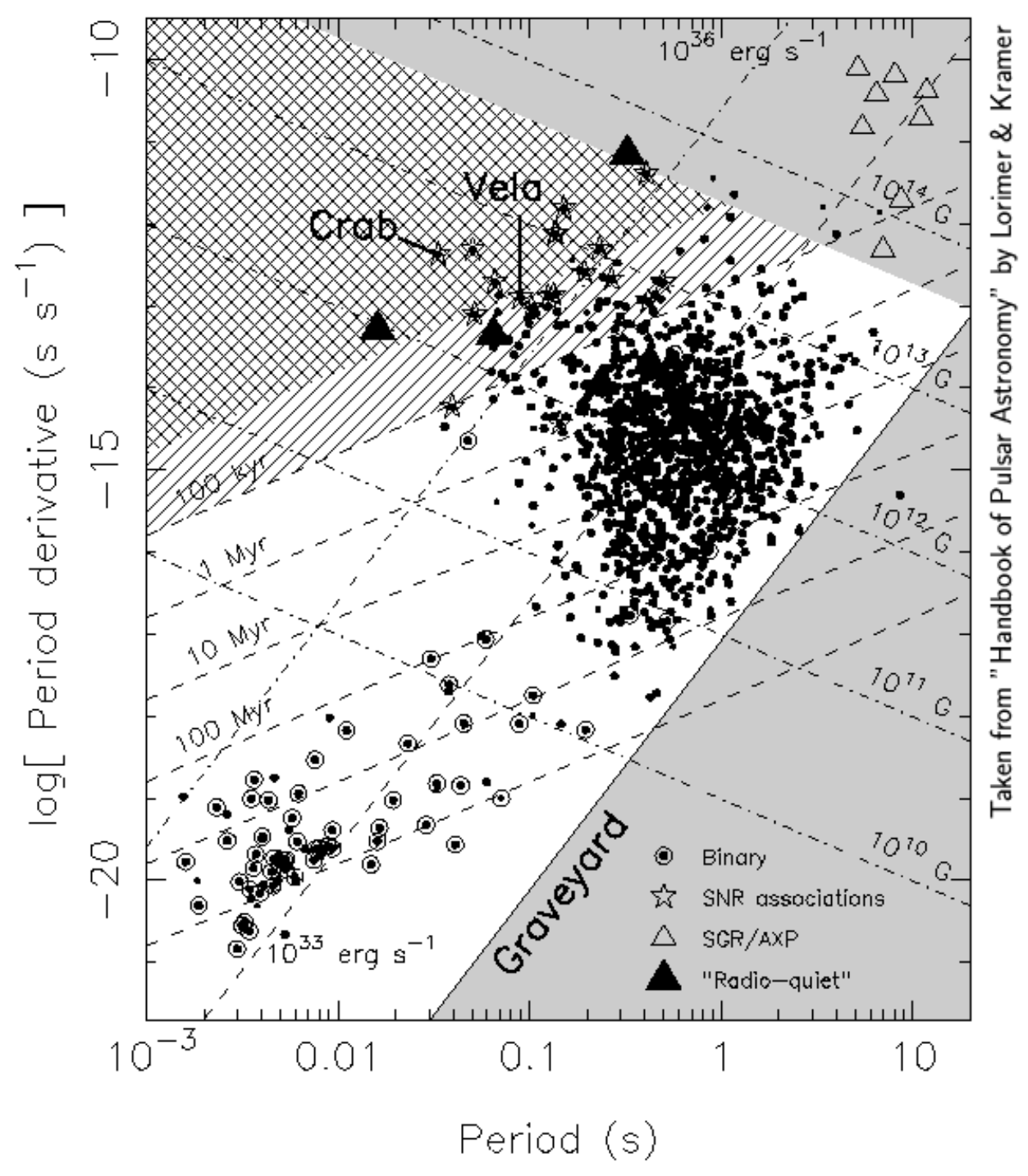

Fig. 1.2. - The $P-\dot{P}$ diagram shown for a sample consisting of radio pulsars, 'radioquiet' pulsars, soft-gamma repeaters (SGRs) and anomalous X-ray pulsars (AXPs). Pulsars known to be members of binary systems are highlighted by a circle. Pulsarsupernova remnant associations are shown by the stars. Lines of constant characteristic age $\tau$, dipolar magnetic field $B$ and spin-down luminosity $\dot{E}$ are also shown. The single hashed region shows 'Vela-like' pulsars with ages in the range 10-100 kyr, while the double-hashed region shows 'Crab-like' pulsars with ages below $10 \mathrm{kyr}$. The grey regions are areas where radio pulsars are not predicted to exist by theoretical models. Figure and caption are from Lorimer \& Kramer (2004). 
Most pulsars are very weak radio sources and thus the coherent addition of many hundreds of pulses (a process, known as folding) is required to obtain a discernable pulse profile.

The integrated pulse profile varies in shape and complexity from pulsar to pulsar, but remains (with a few exceptions) very stable in time. However, the pulse profile often changes its shape with the observing frequency - the number, relative intensity, and position of components can vary.

Pulsars are among most polarized of all known radio sources - the average degree of linear / circular polarization is about $20 \% / 10 \%$. Some pulsars are completely polarized. Normally the position angle of linearly polarized flux varies smoothly across the pulse and the shape of the curve does not depend on the observing frequency. The fits to position angle versus pulse phase allow one to constrain the configuration of the magnetic field in the magnetosphere, under the assumption that radio emission is polarized in the plane of the curvature of the magnetic field lines. However, some pulsars (especially the millisecond ones) show severe deviations from smooth position angle behavior.

The stable and smooth integrated profile is a sum of individual pulses which exhibit a rich diversity of behavior among themselves (see Fig. 1.3). The components of an individual pulse are often identifiable as characteristic 'subpulses' with a width of roughly one third of the average pulse or less. These subpulses may occur at apparently random longitudes within the average profile, or show preference towards certain longitudes (at which the integrated profile shows peaks), or they may drift across the window, appearing at longitudes slowly changing with each pulsar period. The subpulses are interpreted as radiation from an isolated location in the pulsar magnetosphere. A recent study showed that for a majority of pulsars the period-averaged subpulse energy obeys a lognormal distribution (Burke-Spolaor et al. 2012). About half of normal pulses have subpulses which consist of a series of well-separated, sharp and intense micropulses. These microsecond-wide pulses appear to be a modulation of subpulse radiation rather than a distinct radiation component. Both subpulses and 


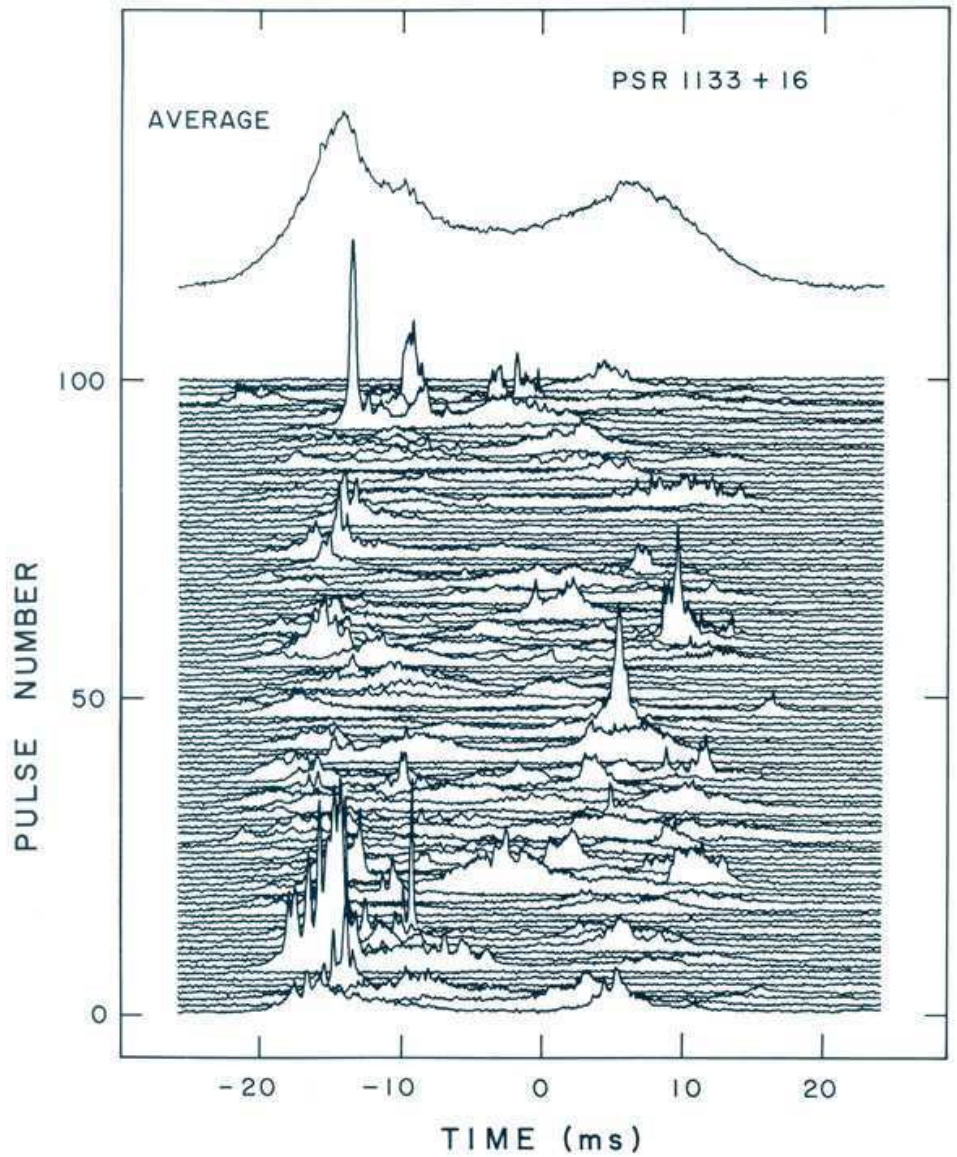

Fig. 1.3. - An example of a pulse sequence together with the integrated pulse profile from a normal pulsar, B1133+16. The figure is from http://arecibo.tc.cornell.edu/PALFA/images.aspx 
micropulses usually show very high degree of polarization.

From some pulsars we detect more than one population of single pulses. For example, B0656+14 has weak broad pulses and bright narrower 'spikes' with clear microstructure (Weltevrede et al. 2006). If a pulsar with such spiky emission is sufficiently far away, it has chances to be detected only through its bright rare pulses, as the signal to noise of the integrated profile will be not high enough. Such pulsars were named Rotating Radio Transients (McLaughlin et al. 2006). The canonical example of 'abnormal' pulses are so-called Giant Pulses (GPs). They were discovered in the 1970s and for a long time were the only abnormal pulse population known. Unlike spiky emission, GPs are very short (from nanoto microseconds) and are detected, with one exception, in narrow phase windows within the average profile. These phase windows are often aligned with the peaks of average profiles at X-ray and $\gamma$-ray energies. GPs have power-law energy distributions and can exceed the average pulse energy $E_{\mathrm{av}}$ by hundreds and thousands of times. Historically, a formal definition of a GP was established as any pulse with $E>10 E_{\mathrm{av}}$. For a long time such pulses were detected from only a few pulsars, the ones with high rotational energy loss rate $\dot{E}$, and the highest magnetic field at the light cylinder. However, recently there have been strong pulses discovered from normal pulsars with ordinary values of $\dot{E}$ and of the magnetic field at the light cylinder. These pulses had been detected at low radio frequencies $(30-300 \mathrm{MHz})$, they have $E>10 E_{\text {av }}$, but their widths and phase of occurrence are somewhat different from GPs (Kuzmin 2006; Ulyanov et al. 2006; Karuppusamy et al. 2011).

Our contemporary notion of pulsar single pulses is very far from complete. The work presented in this thesis aims to throw some light on the following questions:

- What is the mechanism of GP generation?

- Are strong pulses from normal pulsars related to GPs?

- How many types of single pulse emission are there? 
Chapter 2

Correlation of Fermi Photons with High-Frequency Radio Giant Pulses From The Crab Pulsar 


\subsection{Introduction}

The Crab pulsar was discovered by Staelin \& Reifenstein III in 1968 by its remarkably bright giant pulses (GPs). Giant pulses are short (from few ns to few $\mu \mathrm{s}$ ), sporadic bursts of pulsar radio emission (Popov \& Stappers 2007; Hankins et al. 2003). The nature of GPs is far from being clear and even the precise definition of giant pulse had not yet been given (Knight et al. 2006). GPs generally occur only in certain narrow ranges of pulse phase that are often coincident with pulses seen at X-ray and $\gamma$-ray energies (Lundgren 1994). Popov et al. (2006) propose that all radio emission from the Crab (except for that in the precursor) is composed entirely of GPs, consistent with the alignment of the GP and high-energy components seen in other pulsars exhibiting GPs (Cusumano et al. 2003; Knight et al. 2006).

The Crab pulsar shows pulsed emission across the entire electromagnetic spectrum (see Fig. 2.1, left), reflecting different radiation processes in the pulsar magnetosphere - from coherent curvature or synchrotron (radio) to incoherent synchrotron (optical and X-ray) and incoherent curvature ( $\gamma$-ray) radiation. Similar to other sporadic variability phenomena seen in pulsar radio emission, represented by nulling pulsars (e.g. Herfindal \& Rankin 2009), intermittent pulsars (Kramer et al. 2006), and rotating radio transients (McLaughlin et al. 2006), GP emission could be due to changes in the coherence of the radio emission, variations in the pair creation rate in the magnetosphere, or changes in the beaming direction. If the GP phenomenon is due to changes in the coherence of the radio emission mechanism, then one would expect little correlation of the radio GPs with the high-energy emission. However, if the GPs are due to changes in the actual rate of pair creation in the pulsar magnetosphere, one would expect an increased flux at high energies at the time of the GPs. Similarly, because the radio GP and $\gamma$-ray components are aligned, one expects that they come from the same place in the pulsar magnetosphere. Therefore, if a GP occurs from a beam direction alteration, one would expect to also see an increase in the high-energy flux. 

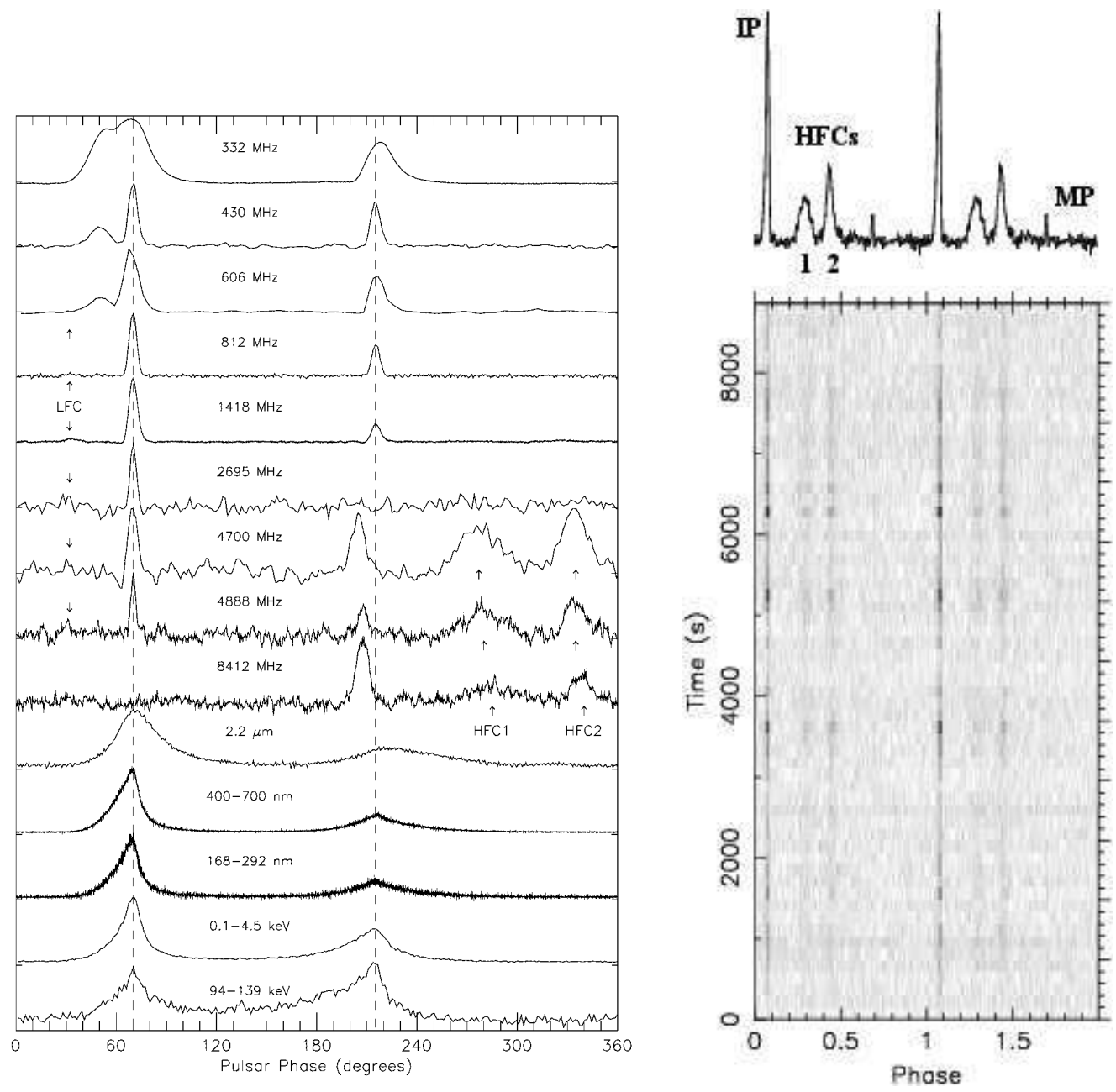

Fig. 2.1. - Left. Average profile of the Crab pulsar from radio to $\gamma$-rays, from the paper of Moffett \& Hankins (1996). Right. Average Crab pulsar radio profile for one out of two sub-sessions with the GBT on Sep 25, 2009. 
Lundgren et al. (1995) previously attempted to carry out simultaneous radio/ $\gamma$ ray observations $(50-220 \mathrm{keV}$, the energy range of CGRO/OSSE) and correlate times of arrival of GPs at 800 and $1300 \mathrm{MHz}$ with $\gamma$-ray photons. Their upper limit on the $\gamma$-ray flux increase concurrent with radio GPs was $\leq 2.5$. Later, Ramanamurthy \& Thompson (1998) correlated the same set of GPs with EGRET photons of energy $>50 \mathrm{MeV}$, placing an upper limit on concurrent $\gamma$-ray flux of 4.6 times the average Crab flux. This suggested that the GP mechanism is largely based on changes in coherence and not changes in pair production rates or beaming. Yet, Shearer et al. (2003) performed simultaneous radio/optical observations of the Crab pulsar and found a weak correlation, i.e. that optical pulses coincident with radio GPs were on average $3 \%$ brighter than others. This observation suggested that the GP emission mechanism, whatever its nature, includes small variations in magnetospheric particle density.

Lyutikov (2007) proposed a more specific, quantitative model of GP emission in which Crab GPs are generated on closed magnetic field lines near the light cylinder via anomalous cyclotron resonance on the ordinary mode. During emission of a photon, an electron undergoes transition up in Landau levels. The energy is supplied by the parallel motion (Ginzburg 1985). The application of anomalous cyclotron resonance to pulsar radio emission has been discussed by Lyutikov et al. (1999) and Machabeli \& Usov (1979).

One clear prediction of this model is that radio GPs (at least those at radio frequencies $>4 \mathrm{GHz}$ ) should be accompanied by $\gamma$-ray photons, as the high energy beam is expected to produce curvature radiation at energies $\sim \hbar \gamma^{3} \Omega \sim$ $0.1-100 \mathrm{GeV}$, depending on the exact value of the Lorentz factor $\gamma$. These energies fall into the energy range of the Fermi mission, and so this hypothesis can also be tested through high-frequency radio observations concurrent with Fermi.

The Fermi Large Area Telescope (LAT), with its large effective area, broad field of view, and superior angular resolution is a perfect tool for testing the Lyutikov theory and investigating the possible correlation between GPs and 
$\gamma$-ray photons in general. For the radio observations, using the 100-m Green Bank Telescope (GBT) allows one to record a very large number of GPs within a reasonable observing time, even at frequencies above $4 \mathrm{GHz}$. Thus, a thorough study of the correlation between high-energy $\gamma$-ray photons and high-frequency GPs is possible.

In this paper, we present the results of simultaneous GBT/LAT observations of the Crab pulsar. To probe the level of correlation between GPs and $\gamma$ ray photons, we used two main approaches. First, we searched for a "burst correlation" by examining whether GPs cluster near $\gamma$-ray photons in time. Second, we analyzed whether the average $\gamma$-ray flux of the pulsar increases within the pulse phase windows where single GPs are produced.

In Sections 2.2 and 2.3 below we describe the radio observations and Fermi data used in this analysis. Section 2.4 discusses the influence of the interstellar medium on the observed GP sample. We describe the correlation analysis between radio GPs and Fermi photons in Section 2.5, and conclude in Section 2.6.

\subsection{Radio observations}

The radio observations were carried out during 12 observing sessions in SeptemberOctober, 2009 with the GBT, using the new Green Bank Ultimate Pulsar Processor Instrument (GUPPI) at a central frequency of $8.9 \mathrm{GHz}$, in incoherent dedispersion mode. The total bandwidth of $800 \mathrm{MHz}$ was split into 256 frequency channels, and the total intensity was recorded with a sampling interval $2.56-3.84 \mu \mathrm{s}$. Total observing time was $\sim 26 \mathrm{hrs}$ or $\sim 3 \times 10^{6}$ pulsar periods.

The raw data from every session were dedispersed with the current DM of the Crab pulsar ${ }^{1}$ using PRESTO package ${ }^{2}$, and searched for all single-pulse events with signal-to-noise ratio $(\mathrm{S} / \mathrm{N})>7$. Since GPs from the Crab pulsar do not

\footnotetext{
${ }^{1}$ The DM was $56.8005 \mathrm{pc} \mathrm{cm}^{-3}$ for September and $56.8109 \mathrm{pc} \mathrm{cm}^{-3}$ for October, from the Jodrell Bank Crab pulsar monthly ephemeris: http://www.jb.man.ac.uk/pulsar/crab.html

${ }^{2}$ http://www.cv.nrao.edu/ sransom/presto/
} 
Table 2.1. Summary of observational parameters and GP/ $\gamma$-ray outcome for each observing date. Columns include (from left to right): date of

observation, time resolution, system equivalent flux density (SEFD), total

duration of radio observations and the time simultaneous with Fermi,

number of giant pulses, $N_{\mathrm{GPs}}$, detected during the whole observing session and during the time simultaneous with Fermi, number of $\gamma$-ray photons, $N_{\gamma}$.

\begin{tabular}{|c|c|c|c|c|c|c|c|}
\hline \multirow{2}{*}{$\begin{array}{l}\text { Date } \\
\text { (2009) }\end{array}$} & \multirow{2}{*}{$\begin{array}{c}\Delta t \\
(\mu \mathrm{s})\end{array}$} & \multirow{2}{*}{$\begin{array}{l}\text { SEFD } \\
(\mathrm{Jy})\end{array}$} & \multicolumn{2}{|c|}{ Radio time } & \multicolumn{2}{|c|}{$N_{\mathrm{GPs}}$} & \multirow[t]{2}{*}{$N_{\gamma}$} \\
\hline & & & $\begin{array}{l}\text { Total } \\
(\min )\end{array}$ & $\begin{array}{l}\text { With Fermi } \\
\text { (min) }\end{array}$ & All & $\begin{array}{l}\text { With } \\
\text { Fermi }\end{array}$ & \\
\hline Sep 12 & 2.56 & 0.81 & 87.1 & 28.9 & 139 & 8 & 5 \\
\hline Sep 14 & 3.20 & 0.73 & 165.7 & 62.1 & 4375 & 1834 & 10 \\
\hline Sep 16 & 3.84 & 0.66 & 99.3 & 30.6 & 98 & 27 & 4 \\
\hline Sep 19 & 3.20 & 0.73 & 118.5 & 54.3 & 6957 & 1830 & 5 \\
\hline Sep 20 & 3.20 & 0.73 & 110.5 & 32.2 & 1846 & 384 & 2 \\
\hline Sep 21 & 3.84 & 0.66 & 55.1 & 31.5 & 27 & 16 & 2 \\
\hline Sep 22 & 3.20 & 0.73 & 147.7 & 68.9 & 1256 & 603 & 5 \\
\hline Sep 23 & 3.20 & 0.73 & 164.5 & 82.0 & 10520 & 5078 & 10 \\
\hline Sep 24 & 3.84 & 0.66 & 55.3 & 22.5 & 38 & 37 & 1 \\
\hline Sep 25 & 3.20 & 0.73 & 236.5 & 130.8 & 14320 & 10014 & 13 \\
\hline Sep 28 & 3.20 & 0.73 & 72.3 & 48.1 & 34 & 9 & 5 \\
\hline Oct 25 & 3.20 & 0.73 & 157.6 & 41.3 & 3164 & 1261 & 15 \\
\hline Total & & & 1470.0 & 633.1 & 42774 & 21092 & 77 \\
\hline
\end{tabular}


have any established lower limit on peak flux density (Popov et al. 2006), we picked up initial threshold of $\mathrm{S} / \mathrm{N}>7$ in order not to contaminate our sample by numerous spurious detections on noise. Each event was assigned a width, found by averaging the dedispersed time series with different numbers of samples and finding the number that resulted in a peak in $\mathrm{S} / \mathrm{N}$. The list of event times was put into TEMPO2 format (Hobbs et al. 2006) and converted to the barycentric reference frame for the correlation analysis with Fermi data. Times of arrival (TOAs) were corrected for delay due to propagation in the ionized interstellar medium (ISM).

Estimated timing errors due to an inaccurate DM are less than our time resolution, assuming that DM varies smoothly and that between observing sessions the change in DM is less than the change over two months. For $\mathrm{DM}_{\mathrm{Oct}}-\mathrm{DM}_{\mathrm{Sep}}=$ $0.0104 \mathrm{pc} \mathrm{cm}^{-3}$, timing errors are about $0.5 \mu \mathrm{s}$.

Fig. 2.1 (right) shows the average pulse profile (top) of the Crab pulsar at 8.9 GHz together with the subintegrations from one of the two sub-sessions on Sep 25, 2009, which had the highest rate of GP detection of all 12 sessions. The interpulse (IP) and high-frequency components (HFCs) are clearly seen, with the weak peak after HFC2 being the main pulse (MP). However, on Sep 25 the pulsar was the brightest, and during other sessions the average profile was less prominent. During some sessions we did not accumulate a detectable average profile at all.

The system equivalent flux density (SEFD) is mostly determined by the Crab Nebula. Flux densities for the Crab Nebula were calculated with the relation $S(f)=955 \times(f / G H z)^{-0.27} \mathrm{Jy}$ (Cordes et al. 2004), accounting for the fact that at $8.9 \mathrm{GHz}$ the solid angle of the GBT beam covers only $25 \%$ of the area occupied by the nebula. We estimate a SEFD $=1.3 / \sqrt{\Delta t / 1 \mu \mathrm{s}} \mathrm{Jy}$, or about $0.7 \mathrm{Jy}$ for our most common sampling time, or $\Delta t$, of $3.2 \mu \mathrm{s}$.

Preliminary analysis of all events with $\mathrm{S} / \mathrm{N}>7$ revealed that GPs appear mostly in the MP and IP phase windows. Only for 2 observing sessions, namely on Sep 25 and 28, were there several GPs detected in the HFCs, all with S/N close to 
9. This is to some extent surprising, since Jessner et al. (2005) observed the Crab pulsar with similar parameters and timespan, and found about 120 GPs in HFCs versus 350 GPs in the MP and IP. However, their threshold peak flux density of $25 \mathrm{Jy}$ was much higher than our threshold of about $6 \mathrm{Jy}$, suggesting that the GPs from HFCs are rarer but brighter.

Some of our sessions were heavily contaminated with broad radio frequency interference $(\mathrm{RFI})$ pulses with typical $\mathrm{S} / \mathrm{N}<10$. Therefore, we analyzed only events with a peak flux density exceeding 8.1 Jy ( $\mathrm{S} / \mathrm{N}=10$ for the session with smallest sampling time) and which arrived in the MP or IP phase windows. Additionally, we excluded all events with width larger than 30 sample intervals, as being presumably caused by RFI. These cuts resulted in the selection of more than 40000 GPs. Comparing the number of single pulses above 8.1 Jy and narrower than 30 samples in and out of the pulsed emission phase windows, we can estimate the proportion of false GPs in our final data set to be less than $0.001 \%$.

The summary of observations is given in Table 2.2. For each observing date the listed columns are: time resolution, SEFD, total duration of radio observations and the time simultaneous with Fermi, number of giant pulses, detected during the whole observing session and during the time simultaneous with Fermi, and the number of $\gamma$-ray photons selected for further analysis (see Section 2.3).

\section{$2.3 \quad$ Fermi data}

We extracted "Diffuse" class events with energies from $100 \mathrm{MeV}$ to $300 \mathrm{GeV}$ from the Fermi database, concurrent with each radio observation. Photons with zenith angles greater than $105^{\circ}$ were excluded to eliminate the $\gamma$-rays generated in the Earth's atmosphere.

We selected only photons in Good Time Intervals (GTIs) within an angle $\theta<$ $\operatorname{Max}(6.68-1.76 \log (E / 1000 \mathrm{MeV}), 1.3)^{\circ}$ of the radio pulsar position (Abdo et al. 2010). Photon arrival times were converted to the Solar System barycenter and 
assigned phases with the TEMPO2 fermi plugin. The timing accuracy of the Fermi LAT is better than $1 \mu \mathrm{s}$ (Abdo et al. 2010). LAT dead time per event is less than $100 \mu \mathrm{s}^{3}$, which is less than $3 \%$ of pulsar rotational phase. Over the course of all radio observations we accumulated 10.5 hours of Fermi data within GTIs, resulting in 77 photons with energies above $100 \mathrm{MeV}$ (see Table 2.2).

Fig. 2.2 gives a quick visual summary of our simultaneous observations, showing Fermi photons and radio GPs versus observing time for each session. The distribution of number and energy/peak intensity of photons/GPs with respect to pulsar rotational phase is shown in Fig 2.3. As reported earlier, $\gamma$-ray and radio emission windows are aligned.

\subsection{Propagation effects for Giant Pulses}

At high frequencies, GPs are strongly affected by interstellar scintillations (Cordes et al. 2004), which change their apparent rate and peak intensities. Careful treatment of ISM effects is crucial for a proper correlation analysis. Unfortunately, we did not make direct measurements of typical ISM diagnostic parameters, such as scintillation bandwidth and pulse broadening time. Instead, we use the scintillation timescales scaled from other frequencies. These values give only a rough estimate of scintillation timescales at $8.96 \mathrm{GHz}$, since for the Crab pulsar the main contribution to scintillations is made by the turbulent and quickly changing Crab Nebula. Scintillation parameters for the Crab have been shown to be strongly variable with time (see Cordes et al. (2004) and references therein).

The refractive interstellar scintillation (RISS) timescale, $\tau_{\text {RISS }}$, can be scaled using the $\nu^{-2.2}$ dependance derived from a five frequency dataset (Rickett \& Lyne 1990). At $8.96 \mathrm{GHz}, \tau_{\text {RISS }}$ is about 80 minutes, thus roughly matching the observed day-to-day variation of the GP rate (see Fig. 2.2). Also, for the

\footnotetext{
${ }^{3}$ The dead time was taken from Fermi Technical Handbook, http://fermi.gsfc.nasa.gov/ssc/proposals/manual/
} 
longest, 4-h session, note the change of GP rate between two 2-h scans in Fig. 2.2 (observing settings were the same for both scans).

Following Cordes et al. (2004), for calculating the diffractive interstellar scintillation timescale, $\tau_{\text {DISS }}$, we adopted the thin screen model with a Kolmogorov spectrum of irregularities and reference pulse broadening time $\tau_{d}=0.5 \mathrm{~ms}$ at $0.3 \mathrm{GHz}$. At $8.9 \mathrm{GHz}$, with bandwidth of $800 \mathrm{MHz}$, this gives us a scintillation strength $u=8.9$, well into the strong scintillaton regime, and $\tau_{\text {DISS }}=\tau_{\text {RISS }} / u^{2} \simeq$ $9 \mathrm{~min}$.

The variation of the GP rate within each observing subsession was estimated by autocorrelating the rate of GP emission in 10-second bins. The autocorrelation analysis shows two shorter GP rate variability time scales of $\sim 20$ minutes and 2-4 minutes. Both of them agree fairly well with the DISS estimations, considering all the uncertainty in the scintillation parameters. On the other hand, there is no evidence against the hypothesis that at least one of these timescales is due to intrinsic GP rate variability.

The following analysis assumes that observed day-to-day variation (or, in case of Sep 25, variation between 2 subsessions) of the GP rate and mean intensity is caused by RISS. If intrinsic GP rate and mean intensity are constant on timescales larger than $90 \mathrm{~min}$, then it is relatively easy to make a GP sample corrected for refractive scintillation. We accomplish this by estimating the amount of RISS intensity damping on each observation session with respect to the session with the highest GP rate. Then, we multiplied the intensities of all pulses in each separate session by those amounts, and threw out all GPs below a threshold, common for the corrected GPs over all sessions.

A simple way to calculate the intensity variation due to RISS would be by comparing mean profiles of pulsed emission accumulated during each session. However, at these frequencies, our observations were not sensitive enough to accumulate the normal Crab pulse profile except on one or two sessions where scintillations caused a boosting of the average flux density of the pulsar. Instead, we compared the intensity distributions of GPs between sessions. If the change 
in rate and mean intensity of GPs on timescales of a few hours is due to RISS, then the peak intensity distributions for each day should have the same shape, but with different values of peak flux density. The distributions in Fig. 2.4 (left) show that this assumption is basically correct.

As the reference session, we picked the one with the highest rate of GPs, the second subsession of Sep 25 (from now on called "0925/2" or "reference session"). For each day and for the two subsessions on Sep 25 separately, we determined the RISS damping coefficient, $k$, by minimizing $\chi^{2}$ :

$$
\chi_{k}^{2}=\frac{1}{N_{\mathrm{bins}}-1} \sum_{I_{i}} \frac{\left[N_{\mathrm{ref}}\left(I_{i}\right)-N\left(I_{i} / k\right)\right]^{2}}{\sigma_{N\left(I_{i} / k\right)}^{2}},
$$

where $N\left(I_{i}\right)$ is the number of GPs with peak intensity higher than $I_{i}$, per hour of observation. $N_{\text {bins }}$ is the number of bins in the distributions being compared, and $\sigma_{N\left(I_{i} / k\right)}=\sqrt{N\left(I_{i} / k\right) / T_{\mathrm{hrs}}}$ is the Poisson error in each bin if $T_{\mathrm{hrs}}$ is the duration of the session in hours and assuming that the energy of each GP does not depend on the energy of the preceding one.

The $\chi_{k}^{2}$ curves are plotted in Fig. 2.4 (right). All but one have a sharp minimum of $\chi_{k}^{2} \lesssim 10$, indicating reasonable fits, at $k$ s between 1 and 10 . The only outlier is the session of Sep 14th, with $\chi_{\min }^{2}=21.7$, which has an abnormal excess of intrinsically strong GPs (see Fig. 2.2). These GPs do not exhibit any other peculiar properties, other than relatively high peak flux density.

Both RISS coefficients and the corresponding $\chi_{\min }^{2}$ for each day are listed in Table 2.2. We corrected the session GPs by multiplying their flux densities by $k$ and set the intensity threshold for corrected pulses as $8.1 \mathrm{Jy} \times \max (k)=69 \mathrm{Jy}$ (8.1 Jy was our initial threshold, see Section 2.2).

Thus, we effectively selected only those pulses which would have had peak flux density larger than 8.1 Jy if they were observed during the session with highest RISS damping. Our uniform sample of such intrinsically brightest pulses numbered 180 GPs with TOAs within the Fermi observing time. 
Table 2.2. RISS correction coefficients $k$ for each observing session.

\begin{tabular}{lcc}
\hline \hline Session & RISS correction coefficient $k$ & $\chi_{\text {min }}^{2}$ \\
& & \\
\hline Sep 12 & 6.4 & 3.1 \\
Sep 14 & 2.2 & 21.7 \\
Sep 16 & 7.2 & 4.7 \\
Sep 19 & 1.4 & 3.8 \\
Sep 20 & 2.7 & 3.9 \\
Sep 21 & 8.5 & 3.3 \\
Sep 22 & 3.4 & 6.6 \\
Sep 23 & 1.3 & 4.8 \\
Sep 24 & 7.7 & 2.5 \\
Sep 25/1 & 5.0 & 2.0 \\
Sep 25/2 & 1.0 & 0.0 \\
Sep 28 & 8.5 & 1.7 \\
Oct 25 & 2.5 & 5.2 \\
\hline
\end{tabular}




\subsection{Correlation analysis}

The correlation analysis focused on two distinct tasks. The first one aimed to probe if the GP generation rate correlates with observed $\gamma$-ray photons. For the second, we investigated the hypothesis of Lyutikov (2007) that the $\gamma$-ray photon flux increases during GPs. For both cases, we used simulated highenergy datasets with no assumed intrinsic correlation between the GPs and $\gamma$-ray photons to test the statistical level of correlation present in the real data. The simulations used the gtobssim software from the Fermi tools package. We used the latest version of instrument response function, Pass6_v3 together with the same spacecraft/pulsar ephemeris as in real data analysis.

We simulated the pulsar using the PulsarSpectrum library, with the light curve, spectrum and integral flux above $100 \mathrm{MeV}$ taken from Abdo et al. (2010). The integral flux was set to $F_{\text {av }}=2.09 \times 10^{-6} \mathrm{~cm}^{-2} \mathrm{~s}^{-1}$ for the burst correlation analysis and varied from 0 up to about a hundred $F_{\text {av }}$ for the single-pulse correlation analysis (see 2.5.2). When we simulated zero flux from the Crab pulsar, we simply removed the pulsar from the list of simulated sources.

We modeled the Crab Nebula as a point source (for the energy ranges in question its angular diameter is less than the Fermi region of interest), with the spectrum as determined in Abdo et al. (2010) and integral flux above $100 \mathrm{MeV}$ of $9.8 \times 10^{-7} \mathrm{~cm}^{-2} \mathrm{~s}^{-1}$. For the Galactic and extragalactic backgrounds we used the "GalacticDiffuse_v02" and "IsotropicDiffuse_v02" models. The simulated photon files were processed in the same way as the real data.

\subsubsection{Is GP rate correlated with single $\gamma$-ray photons?}

To test if $\gamma$-ray photons are correlated with the GP generation rate, we calculated the distribution of time lags between each photon and all GPs in that photon's GTI. The same procedure was applied to the simulated $\gamma$-ray datasets, such that if there were any clustering of GPs around $\gamma$-ray photons (or with some 
time lag with respect to the $\gamma$-ray photons), it would be seen as a discrepancy between the real and simulated distributions of the high-energy data. Changing the bin size of the distribution makes it sensitive to different timescales of possible clustering of GPs. In this study, we tried a set of bin widths, starting from 10 seconds and increasing the width by 10 seconds up to 2 minutes. Two minutes corresponds to the smallest timescale of GP clumping (likely caused by interstellar scintillation), as shown in Fig. 2.2. On timescales less than $10 \mathrm{~s}$, the Poisson noise due to a discrete number of time lag measurements becomes too high. We performed 1000 simulation runs and contrasted the real-data distribution with the mean and $95 \%$ percentile of all of the simulated datasets. Fig. 2.5 shows the distribution of time lags between GPs and photons for all GPs and for a RISS-corrected sample of GPs for one particular bin width, namely, 2 min. In both cases the real dataset lies all within the $95 \%$ percentile of the simulations, indicating no apparent change in GP generation rate on 2min timescales with any possible time lag within \pm 40 minutes (maximum GTI length) of the arrival of the $\gamma$-ray photons. All other bin widths, down to $10 \mathrm{~s}$, gave the same result.

\subsubsection{Does $\gamma$-ray flux change around single GPs?}

Another question of interest is whether the average $\gamma$-ray flux from the Crab pulsar increases during individual giant pulses, as predicted by Lyutikov (2007). To investigate that, we looked for the number of $\gamma$-ray photons in on-pulse emission windows around each GP. We performed a separate search for all GPs, looking for photons in a large window consisting of the main pulse, interpulse and bridge between them, and also for IP GPs only, limiting the correlation window to the interpulse phase range.

If a photon was detected in a window around a GP, it was called a "match". For 10.5 hours of simultaneous observations, we detected only one such match: a photon with $E=403.7 \mathrm{MeV}$ was detected within $1.3 \mathrm{~ms}$ of IP GP with peak 
flux density of $8.9 \mathrm{Jy}$. Nonetheless, knowing the observed number of matches $N=1$, the probability that the $\gamma$-ray flux during GPs is equal to some value $F_{0}$ can be estimated with the simple Bayesian formula:

$$
p\left(F=F_{0} \mid N\right)=\frac{p\left(F_{0}\right) \cdot p\left(N \mid F=F_{0}\right)}{\int_{0}^{F_{\text {max }}} p\left(F_{0}\right) \cdot p\left(N \mid F=F_{x}\right) d F_{x}}
$$

where $p\left(F_{0}\right)$ is the prior distribution for $F_{0}$ and $p\left(N \mid F=F_{0}\right)$ is the likelihood, i.e. the probability to get the observed number of matches $N$ if the pulsar $\gamma$-ray flux during GPs is equal to $F_{0}$.

Since little is known about $p\left(F_{0}\right)$, the prior distribution for $F_{0}$, we chose the prior to be uniform in a flux range from 0 (the Crab pulsar turns off $\gamma$-ray emission during GPs) to

$$
F_{\max } \equiv F_{\text {av }} \frac{\text { observing timespan }}{N_{\mathrm{GP}} \times \text { length of window }}
$$

where $F_{\text {av }}$ is the average pulsed $\gamma$-ray flux from the Crab pulsar. $F_{\max }$ corresponds to the hypothesis that all $\gamma$-ray photons from the Crab pulsar come during GPs. For our choice of windows, $F_{\max }$ ranged from $60 F_{\text {av }}$ (for on-pulse phase window) to $150 F_{\text {av }}$ (for IP window).

The likelihood $p\left(N \mid F=F_{0}\right)$ was calculated by running simulations with different pulsed flux $F_{0}$ and computing the fraction of runs with a number of matches $N$. The grid of trial flux values, in units of $F_{\text {av }}$, was as follows: from 0 to 1 with the step of 0.25 , from 1 to 20 with the step of 1 or 0.5 , and then from 20 to 30 with the step of 5 . For both choices of correlation window the probability density went down to 0 before $30 F_{\text {av }}$. Here we implicitly assumed that a higher flux outside selected windows does not influence the correlation within windows. Since the number of simulation runs for each trial $F_{0}$ is finite, it leads to an uncertainty in estimating the likelihood. We estimated the statistical errors from the simulation using the following method. Suppose that for some value of $F_{0}$ we have run $n$ simulations with $y$ successes (i.e. cases where the number 
of matches in the simulation equals the one obtained for real data, $N)$. Then $y / n$ defines the estimate of probability of success $p$, which is also the likelihood density $p\left(N \mid F=F_{0}\right)$. More precisely, $p \mid y$ has a Beta distribution, with mean $(y+1) /(n+1)$ and variance $\sigma_{p}^{2}=\frac{(y+1)(n-y+1)}{(n+3)(n+2)^{2}}$. We adopted $\sigma_{p}$ as an error of $p$ due to limited numbers of simulations performed.

However, there is another major source of uncertainty connected to the fact that we record discrete number of photons around the GPs. Since photon detection is very well described as a Poisson process (Ramanamurthy \& Thompson 1998), the error on detecting $N$ photons in a certain window around GPs will be $\sqrt{N}$. In our case, for all windows we had $N=1$, so to estimate the true value of the likelihood we should take into consideration also the likelihood curves for $N=0$ and $N=2$. These estimates for both windows are plotted in Fig. 2.6, left. The shaded region around each curve corresponds to $\pm \sigma_{p}$, calculated by the above formula. For both correlation windows $N=1$ likelihood curves have maximum around $F_{0} / F_{\text {av }}=1$, which means that, most probably, pulsed $\gamma$-ray flux does not change during GPs (no correlation), or changes no more than few times (weak correlation).

With our limited dataset we cannot say anything more about the exact value of $\gamma$-ray flux during GPs, but we can place upper limits on it. On the grid of simulated fluxes $F_{i}$, one can convert the continuous formula for posterior probability density (eq. 2.2) into a discrete one for the probability that pulsed flux around GPs is less than $F_{0}$ :

$$
P\left(F \leq F_{0} \mid N\right)=\frac{0.5 \cdot \sum_{F_{i+1} \leq F_{0}}\left(p_{i}+p_{i+1}\right)\left(F_{i+1}-F_{i}\right)}{0.5 \cdot \sum\left(p_{i}+p_{i+1}\right)\left(F_{i+1}-F_{i}\right)},
$$

where $p_{i} \equiv p\left(N \mid F=F_{i}\right)$.

To estimate errors in $P\left(F \leq F_{0} \mid N\right)$, we assumed that our uncertainty in $p_{i}$ due to a limited number of trials is much larger than the error from calculating the integral as a sum. As one can see in Fig. 2.6, left, this simplification is reasonable. Assuming all $\sigma_{p_{i}}$ are independent, the uncertainty in $P\left(F \leq F_{0} \mid N\right)$ 
is determined by standard error propagation.

In Fig. 2.6 we show the resulting probabilities that the $\gamma$-ray flux from the Crab pulsar during GPs does not exceed a given number of times the mean flux reported by Abdo et al. (2010). Errors due to the limited number of simulation runs are plotted as errorbars, whereas those due to a discrete number of GP/photon matches are given by separate posterior probability curves for $N=0$ and $N=2$.

Obviously, the smaller the correlation window for a fixed observation timespan and the smaller the number of GPs in the sample, the larger $F_{\max }$, and the broader is the resulting posterior probability density. That is why for our dataset we could obtain the posterior probability densities only for correlation windows which included the IP, because most of GPs come within this phase range. For the main pulse GPs, $p\left(F=F_{0} \mid N\right)$ is very broad, having almost the same probability density up to $\sim 100 F_{\mathrm{av}}$. For the same reasons, the analysis on the sample of GPs corrected for refractive scintillation did not give any meaningful results.

\subsection{Conclusions}

No obvious correlation was found between Fermi photons of energies $>100 \mathrm{MeV}$ and radio giant pulses at the frequency of $8.9 \mathrm{GHz}$. No change in the Crab GP generation rate was found on timescales from 10 to $120 \mathrm{~s}$ around $\gamma$-ray photons and with any possible lag within $\pm 40 \mathrm{~min}$ with respect to $\gamma$-ray photons.

With $95 \%$ probability, the high energy flux of the Crab pulsar during GPs is less than 4 times the average $\gamma$-ray pulsed flux for the on-pulse $(\mathrm{MP}+\mathrm{IP}+$ bridge between them) phase window. For IP GPs only, the $95 \%$ upper limit on $\gamma$-ray flux in the IP phase window is 12 times the average pulsed flux. If we consider the uncertainty due to discrete numbers of matches between photons and GPs, the $95 \%$ upper limits are 3-5.5 times the average pulsed flux for the pulsed emission window, and 8-16 for the IP window. 
A few explanations may be offered for the non-detection of GP- $\gamma$-photon correlations. The most natural is that production of GPs depends on non-stationary changing coherence conditions, which vary by a large degree even for similar magnetospheric particle densities. Another possibility is that beaming in radio and at high energies are somewhat different, so that simultaneous GPs and $\gamma$-ray photons are emitted in different directions.

Overall, our results suggest that enhanced pair creation is not a dominant factor for GP occurrence, at least for high frequency IP GPs. However, our flux increase estimations are not on the level of a few percent, as in the work of Shearer et al. (2003) at optical wavelengths. To reach that sensitivity we need more data, which will help push down the upper limit on flux during GPs and will make possible the analysis on subsamples of GPs, such as the brightest ones. Also, including radio frequencies below $4 \mathrm{GHz}$ is potentially interesting not only for investigating the correlation for MP GPs separately (MP GPs are much more common at lower frequencies), but also for re-doing the analysis for low-frequency IP GPs, since they might be generated by different physical processes than the high-frequency IP GPs (Moffett 1997). All these questions are being investigated with our ongoing radio observation campaign using the 42-ft telescope at the Jodrell Bank Observatory (UK) and the 140-ft telescope at the Green Bank Observatory (WV). 


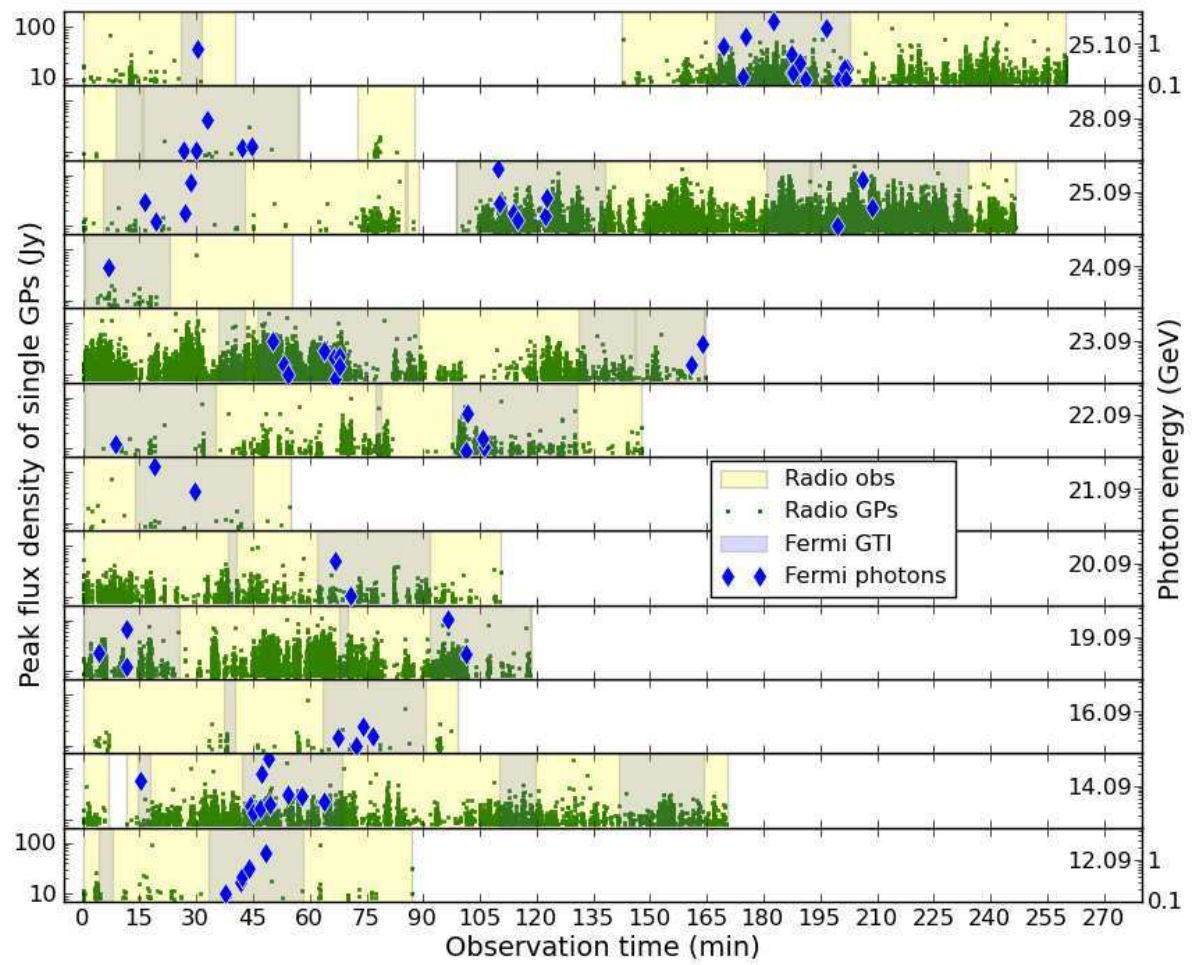

Fig. 2.2.- Time series of radio GPs and Fermi photons during 12 observing sessions. $\mathrm{X}$-axis - time from the beginning of each session, in minutes. Y-axis (left) - peak flux density of radio GPs. Y-axis (right) - energy of $\gamma$-ray photons. Both scales are the same for each observing session. The yellow shaded regions mark the time when we actually were recording radio data and the blue are the Fermi Good Time Intervals. The observing date is given in the upper right-hand corner of each subplot. For the observing session on Oct 25 two photons came within short time interval, so their markers overlap and one can see only 14 photons, instead of 15. 

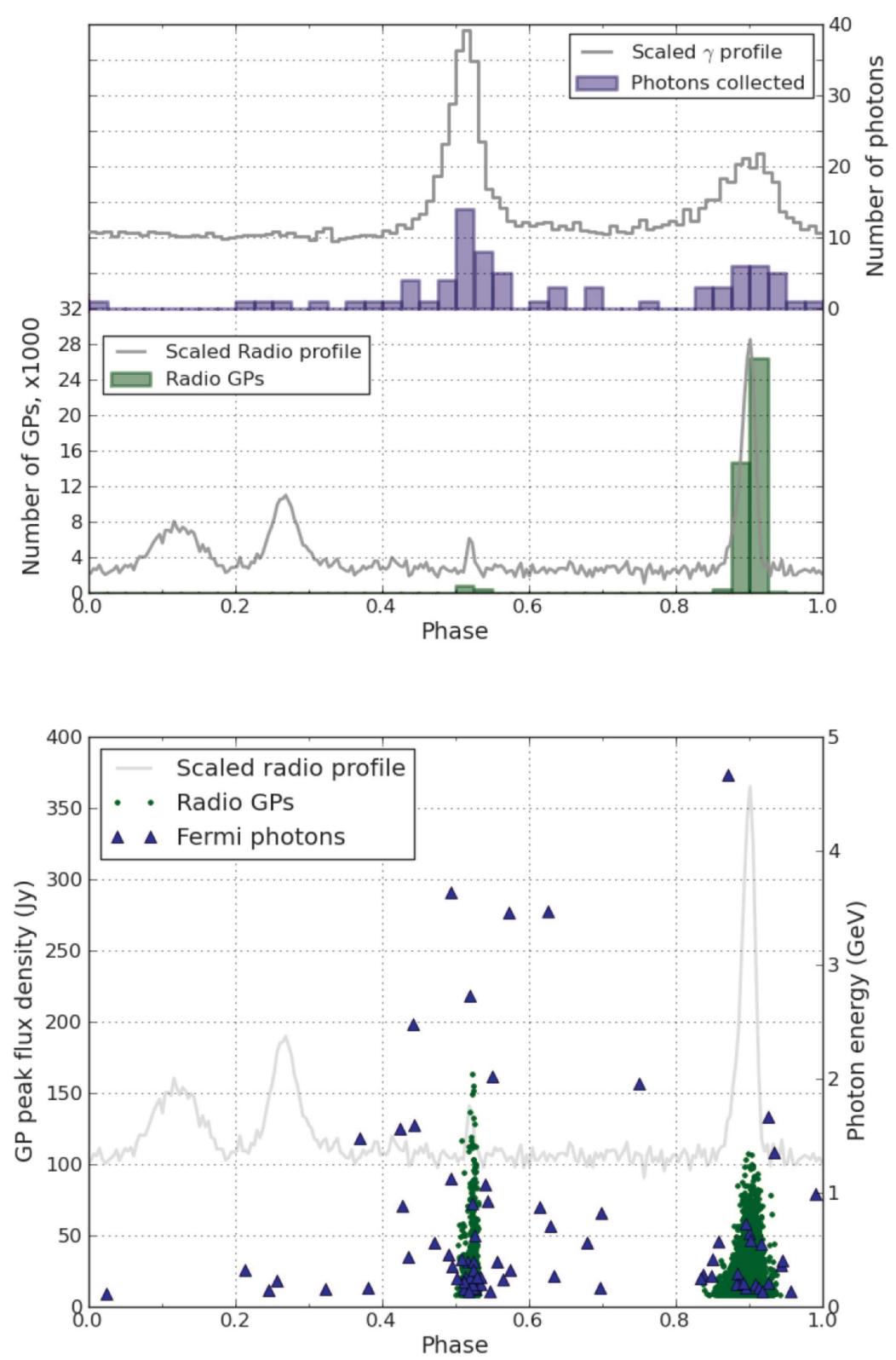

Fig. 2.3. - Top: histograms of GPs for all radio observing time (bottom) and Fermi photons during the simultaneous time (top). For illustrative purposes, scaled radio and $\gamma$-ray profiles (grey) are shown with arbitrary offset along y-axis. The scaled radio profile is from the second scan of GBT session on Sep 25, 2009 (as shown in Fig. 2.1), and the $\gamma$-ray profile is the Fermi profile accumulated during Sep-Oct, 2009. Bottom: distribution of peak flux density of GPs and energy of $\gamma$-ray photons over pulsar rotational phase. Also, there is shown the scaled radio profile as for histogram on the left. 

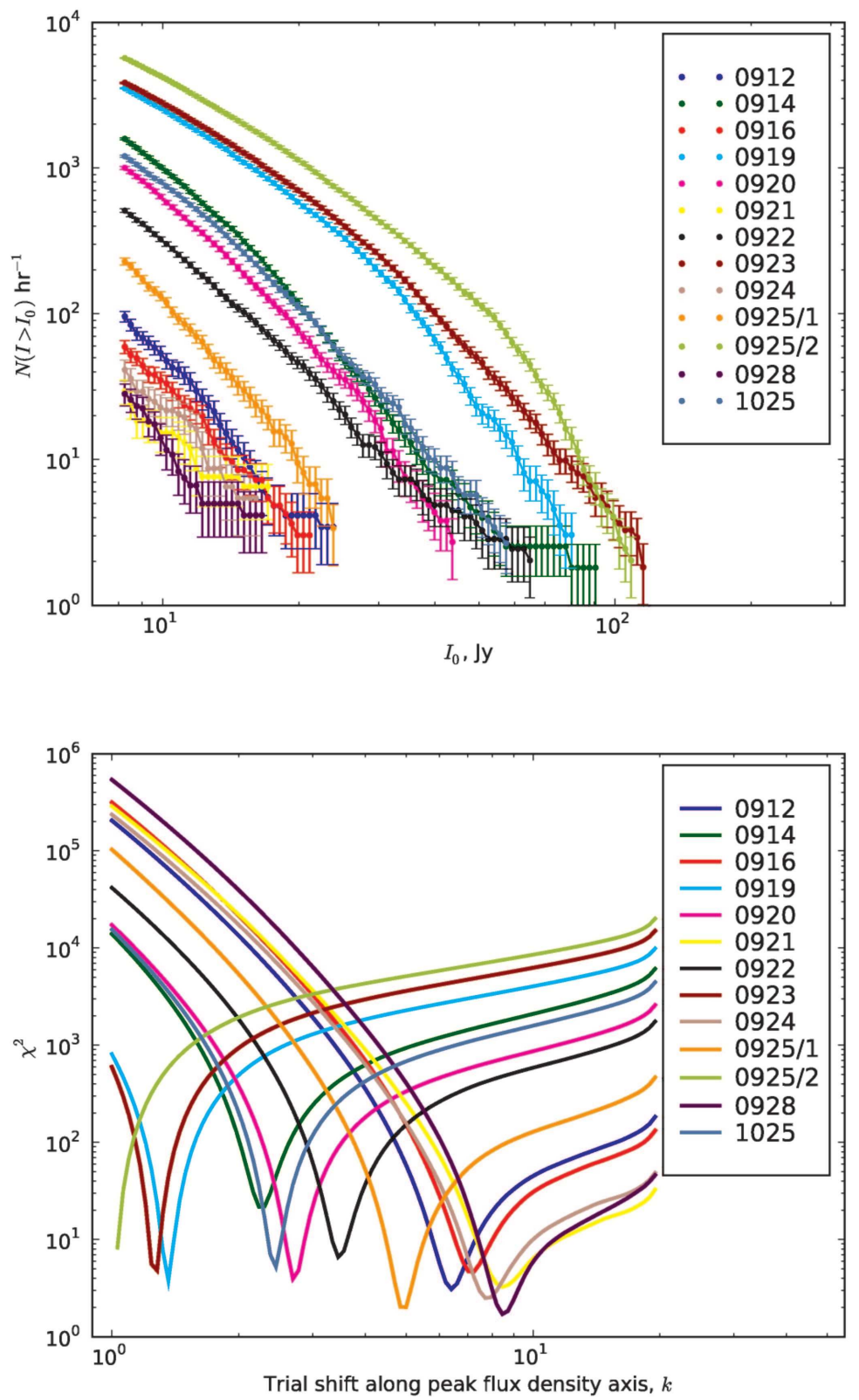

Fig. 2.4. - Top: cumulative peak flux distribution of GPs (MP and IP together) for different observing sessions and 2 subsessions of Sep 25 with apparently different GP rates. Poisson errors are also shown. Bottom: $\chi^{2}$ values versus the RISS damping coefficient, $k$, for each observing session. 

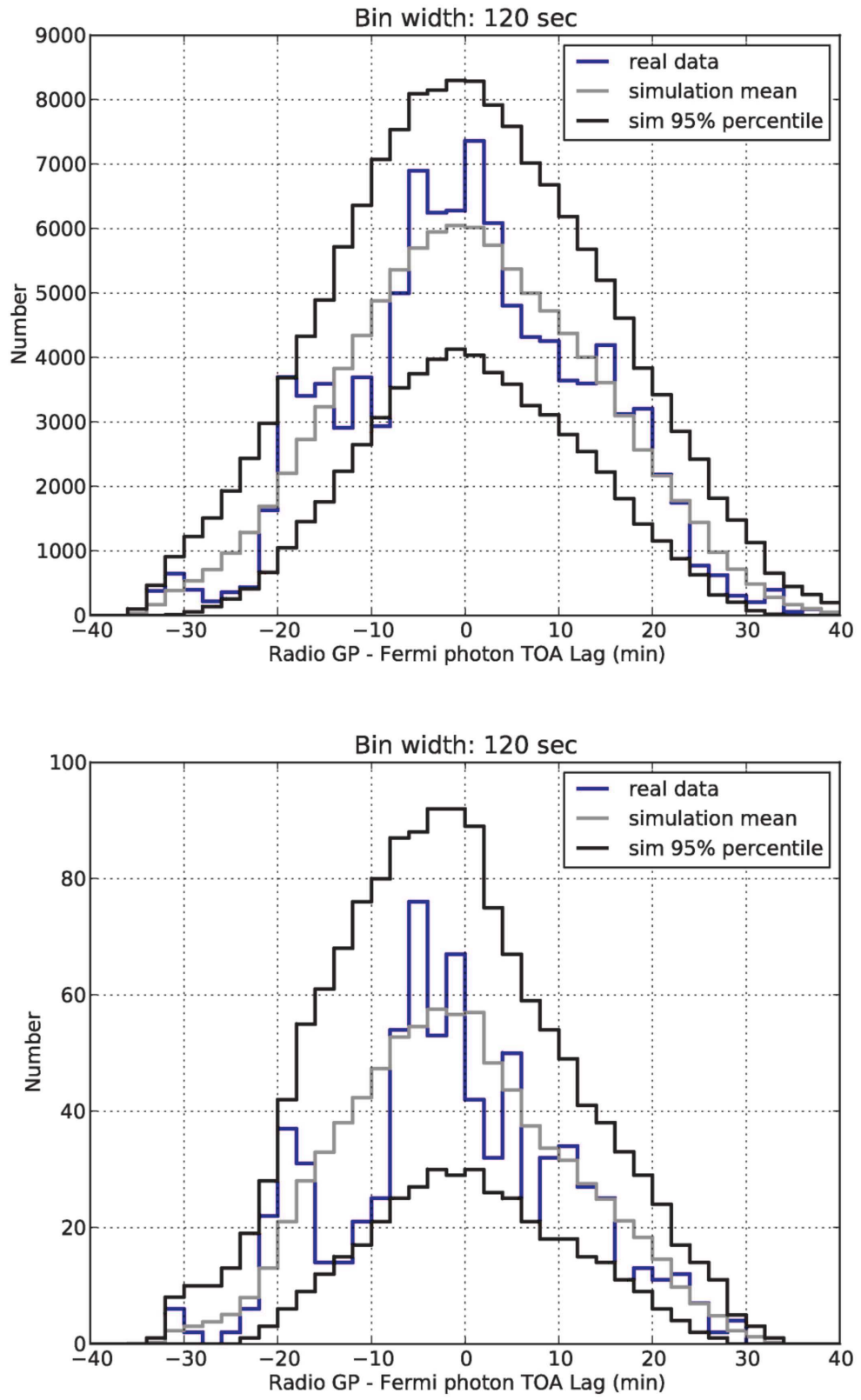

Fig. 2.5. - The distribution of time lags between GPs and $\gamma$-ray photons for 2-min bins, for all GPs > 8.1 Jy (21000 GPs; left) and the RISS-corrected GPs (180 GPs; right). Real Fermi photons (blue line) are contrasted to the mean and $95 \%$ percentile on the pool of simulated datasets (grey and black). The fact that the distribution for the real data lies within $95 \%$ of the simulated ones indicates no apparent change in GP generation rate on 2-min timescale with any possible time lag up to \pm 40 minutes with respect to the $\gamma$-ray photons. The maximum lag value, $40 \mathrm{~min}$, corresponds to the size of the largest GTI window. All other bin widths (down to $10 \mathrm{~s}$ ) give the same result. 

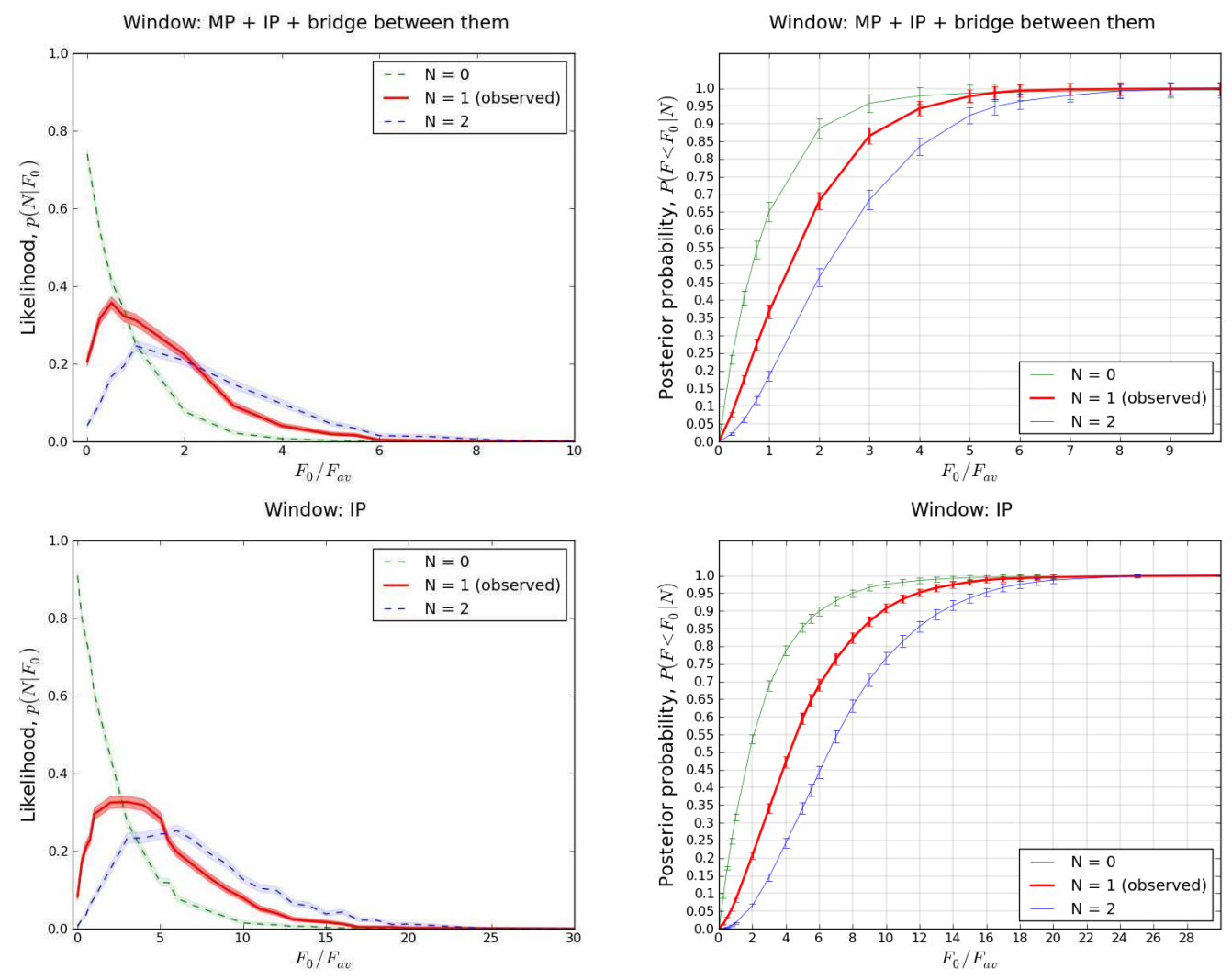

Fig. 2.6. - Left: Likelihood, the probability of observing $N$ photons in a certain window around a GP if the $\gamma$-ray flux from the pulsar in this window were $F_{0}$. For each window size, we observed only one such match between GP and $\gamma$-ray photon, so $N=1$. Since photon arrival times are a Poisson process, the error on $N$ is $\sqrt{N}=1$, thus likelihood curves for $N=0$ and $N=2$ are also shown. The shaded region around each curve corresponds to uncertainty due to the limited number of simulation runs (see text for explanation). Both $N=1$ likelihood curves peak near $F_{0} / F_{\text {av }}=1$, so no or weak correlation between GPs and $\gamma$-ray photons is the most probable. Right: Posterior probability that $\gamma$-ray flux in a window around GP is less than $F_{0}$, given the observed number of matches $N$. Errors due to the limited number of simulation runs are plotted as errorbars, whereas those due to a discrete number of matches are given by separate posterior probability curves for $N=0$ and $N=2 . F_{\text {av }}$, the average pulsed $\gamma$-ray flux from Crab pulsar, is from Abdo et al. (2010). 
Chapter 3

Correlation of Chandra Photons with the Radio Giant Pulses from the Crab Pulsar 


\subsection{Introduction}

Giant pulses are a peculiar form of pulsar radio emission, clearly distinct from the regular pulses. Only a handful of pulsars have been reported to have GPs and neither the nature of GP emission nor the strict definition of a giant pulse are yet fully clear. The "classical" giant pulses, from the pulsars with high values of magnetic field at the light cylinder, are short (ns $-\mu \mathrm{s}$ ), bright (up to MJy) and obey a power-law energy distribution (see Kuzmin 2007, and references therein). These properties obviously point to the coherent nature of the emission mechanism.

It is interesting that for the pulsars with "classical" GPs and pulsed X-rays or $\gamma$-rays, giant pulses come from the same pulse phase range as high-energy emission (Knight 2006; Cusumano et al. 2003). That indicates that GPs and high-energy photons may originate in the same region in the magnetosphere. Without any doubt, the fact that GPs are (or are not) accompanied by increasing high-energy flux is important for testing the theories of GP generation. On a broad scale, if the GP emission mechanism involves some change in particle creation rate or beam direction alteration, one should observe a correlation between GPs and high-energy emission. If GPs are due to changes in coherence in magnetospheric plasma, there should be no correlation, since high-energy emission is of incoherent origin. Also, while propagating in the magnetosphere, radio photons can be shifted to a higher energy band by interacting with the relativistic plasma via inverse Compton scattering or resonant absorption. This would result in potentially detectable radio/high-energy correlation.

The Crab pulsar is the most studied pulsar with GPs and the easiest object for such correlation studies, since the pulsar is relatively bright at all wavelengths. GPs from the Crab appear in phase regions of the main pulse (MP) and interpulse (IP) at all radio frequencies where the Crab has been observed (from $23 \mathrm{MHz}$ up to $15 \mathrm{GHz}$ ). Also, the detection of GPs in high frequency components at $8.35 \mathrm{GHz}$ has been reported by Jessner et al. (2005), albeit not 
Table 3.1. Previous studies of correlation between GPs from the Crab pulsar and its high-energy emission

\begin{tabular}{|c|c|c|c|c|c|}
\hline & $\begin{array}{c}\text { Shearer et al. } \\
\text { (2003) }\end{array}$ & $\begin{array}{c}\text { Lundgren et al. } \\
\text { (1995) }\end{array}$ & $\begin{array}{l}\text { Ramanamurthy \& } \\
\text { Thompson (1998) }\end{array}$ & $\begin{array}{l}\text { Bilous et al. } \\
\quad(2011)\end{array}$ & $\begin{array}{l}\text { Mickaliger et al. } \\
\text { (in prep) }\end{array}$ \\
\hline $\begin{array}{l}\text { High-energy ob- } \\
\text { servation energy } \\
\text { ranoe }\end{array}$ & $6000-7500 \AA$ & $50-220 \mathrm{MeV}$ & $0.05-30 \mathrm{GeV}$ & $0.1-5 \mathrm{GeV}$ & $0.1-300 \mathrm{GeV}$ \\
\hline $\begin{array}{l}\text { Radio obser- } \\
\text { vation } \quad \text { central } \\
\text { frequency }(\mathrm{GHz})\end{array}$ & 1.4 & $1.3,0.8$ & $1.3,0.8$ & 8.9 & $1.2,0.33$ \\
\hline $\begin{array}{l}\text { Radio observa- } \\
\text { tion bandwidth } \\
(\mathrm{MHz})\end{array}$ & 5 & 20 & 20 & 800 & 400,150 \\
\hline $\begin{array}{l}\text { GP selection } \\
\text { threshold (Jy) }\end{array}$ & 150 (peak) & 125 (mean) & 125 (mean) & 8.1 (peak) & $\mathrm{S} / \mathrm{N}>10$ \\
\hline $\begin{array}{l}\text { Observing time } \\
\text { (h) }\end{array}$ & 3 & 14 & 32 & 10 & 30 \\
\hline $\begin{array}{l}\text { High-energy } \\
\text { flux change in } \\
\text { units of aver- } \\
\text { age flux during } \\
\text { observations }\end{array}$ & $\begin{array}{l}3 \% \text { enhanced } \\
\text { flux }(7.8 \sigma) \text { in } \\
0.02 \text { phase win- } \\
\text { dow around MP } \\
\text { GPs }\end{array}$ & $\begin{array}{l}\text { with a } 95 \% \\
\text { confidence level, } \\
<2.5 \text {, in a period } \\
\text { around GPs and } \\
\text { up to } \pm 5 \text { periods }\end{array}$ & $\begin{array}{l}\text { with a } 99.9 \% \\
\text { confidence level, } \\
<4.6 \text { for } 100 \mathrm{~ms} \\
\text { window around } \\
\text { GP }\end{array}$ & $\begin{array}{l}\text { with a } 95 \% \text { con- } \\
\text { fidence level, <4 } \\
\text { for } 30 \mathrm{~ms} \text { around } \\
\text { GPs, <12 for } \\
0.2 \text { phase win- } \\
\text { dow around IP } \\
\text { GPs }\end{array}$ & $\begin{array}{l}\text { maximum } 1 \sigma \\
\text { correlation in } \\
\text { one period } \\
\text { around GPs }\end{array}$ \\
\hline
\end{tabular}


confirmed by later observations (Jessner et al. 2010).

Around $4 \mathrm{GHz}$ the interpulse disappears and then reappears shifted ahead by about 10 degrees (Moffett \& Hankins 1996). Curiously, above 4 GHz IP GPs have totally different spectral properties than MP GPs at the same frequency (Hankins \& Eilek 2007). Thus, IP GPs above $4 \mathrm{GHz}$ may be produced by a separate emission mechanism.

Previously, there have been several correlation studies between Crab GPs and its high-energy emission (see Table 3.1 for summary). The only positive results so far were obtained in the optical light by Shearer et al. (2003). They found that optical pulses coincident with GPs were on average 3\% brighter than the others. A number of $\gamma$-ray studies covering the energy range from $50 \mathrm{MeV}$ to $300 \mathrm{GeV}$ did not find any statistically significant correlation, showing that the $\gamma$-ray flux around GPs does not change by more than a factor of several times (Lundgren et al. 1995, Ramanamurthy \& Thompson 1998, Bilous et al. 2011, Mickaliger et al., in prep).

A few studies have also been conducted of Crab X-ray variability based on highenergy data alone. The analysis of 1 hour of RXTE data (1-10 keV) showed that the flux from the Crab pulsar is stable at the level of 7\% (Patt et al. 1999). Also, in the 13.3 - $58.4 \mathrm{keV}$, based on 18 hours of RXTE data, Vivekanand (2001) found the rms of X-ray flux variation in time at any phase in the onpulse window to be within of $\approx 3.5 \%$ of the corresponding mean flux of the integrated profile of Crab pulsar.

These studies ruled out the existence of short strong "GP-like" bursts of Xray emission, but they were not capable of detecting small sporadic X-ray flux variations, as could be expected around GPs. In this paper we used 5.4 hours of simultaneous observations of the Crab pulsar with the 100-m Robert C. Byrd Green Bank Telescope (GBT) and the Chandra X-ray Observatory to perform an extensive correlation of GPs with X-ray photons. 


\subsection{Radio observations}

Radio observations were carried out on 2010 November 16 with the GBT using the Green Bank Ultimate Pulsar Processing Instrument (GUPPI) at a central frequency of $1.5 \mathrm{GHz}$ in coherent dedispersion search mode. The total nominal band of $800 \mathrm{MHz}$ was split into 128 frequency channels and the full Stokes parameters were recorded with a sampling interval of $t_{\text {res }}=2.56 \mu \mathrm{s}$. Unfortunately, the data from 1.3 to $1.5 \mathrm{GHz}$ were lost because of a backend malfunction, thus reducing the effective bandwidth to $600 \mathrm{MHz}$. Radio observing time comprised 5.4 hours or $5.8 \times 10^{5}$ pulsar periods.

At $1.5 \mathrm{GHz}$ the system temperature is dominated by the Crab nebula, which is smaller (Green 2009) than the 8.2' GBT beam at this frequency. We calculated the flux density for the Crab Nebula with the relation $S(f)=955 \times$ $(f / G H z)^{-0.27} \mathrm{Jy}$ (Cordes et al. 2004). With the receiver temperature $T_{\text {rec }}=$ $20 \mathrm{~K}$, background sky temperature $T_{\mathrm{bkg}}=1.5 \mathrm{~K}$, and gain $G=2 \mathrm{~K} / \mathrm{Jy}$, our estimated system equivalent flux density $\mathrm{SEFD}=7.9 \mathrm{Jy}$ for a $600 \mathrm{MHz}$ bandwidth and a sampling time of $2.56 \mu \mathrm{s}$.

During the observations, we performed coherent dedispersion in each of the frequency channels with the most up-to-date value of dispersion measure (DM) from the Jodrell Bank Crab pulsar monthly ephemeris ${ }^{1}, \mathrm{DM}_{\mathrm{JB}}=56.8065 \mathrm{pc} \mathrm{cm}^{-3}$. However, the dispersion measure of the Crab pulsar is known to vary on monthly timescales by $\sim 0.01 \mathrm{pc} \mathrm{cm}^{-3}$. Such error in DM will not result in detectable pulse smearing within one frequency channel, but across $800 \mathrm{MHz}$ bandwidth the net smearing can reach $20 \mu \mathrm{s}$, or 8 time samples. To check the DM, we looked at the spectra of the brightest events dedispersed with $\mathrm{DM}_{\mathrm{JB}}$. All events exhibited the characteristic quadratic trend across the band, as expected for an error in DM (see Fig. 3.1, left). We dedispersed these events with a set of trial DM values and for each pulse found the DM which corresponded to maximum peak S/N. The mean of these DM values was $56.7963 \mathrm{pccm}^{-3}$ with standard

\footnotetext{
${ }^{1}$ http://www.jb.man.ac.uk/ pulsar/crab.html, see also Lyne et al. (1993)
} 

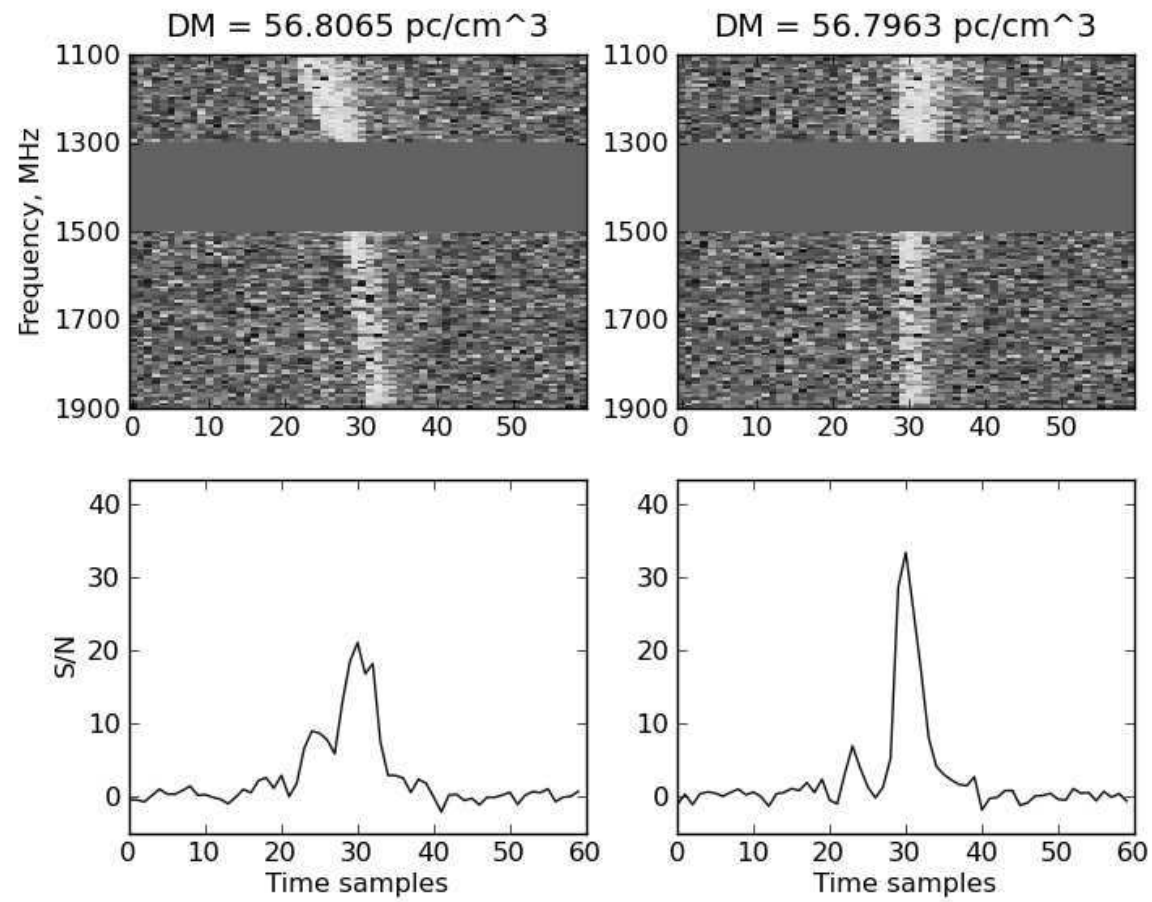

Fig. 3.1. - An example of a strong GP, dedispersed with the DM $=56.8065 \mathrm{pc} \mathrm{cm}^{-3}$ from the Jodrell Bank Crab Pulsar Monthly Ephemeris from Nov 15, 2010 (left) and with the correct DM, corrected by $0.0102 \mathrm{pc} \mathrm{cm}^{-3}$ (right). The gap between 1.3 and $1.5 \mathrm{GHz}$ is due to a backend malfunction. 

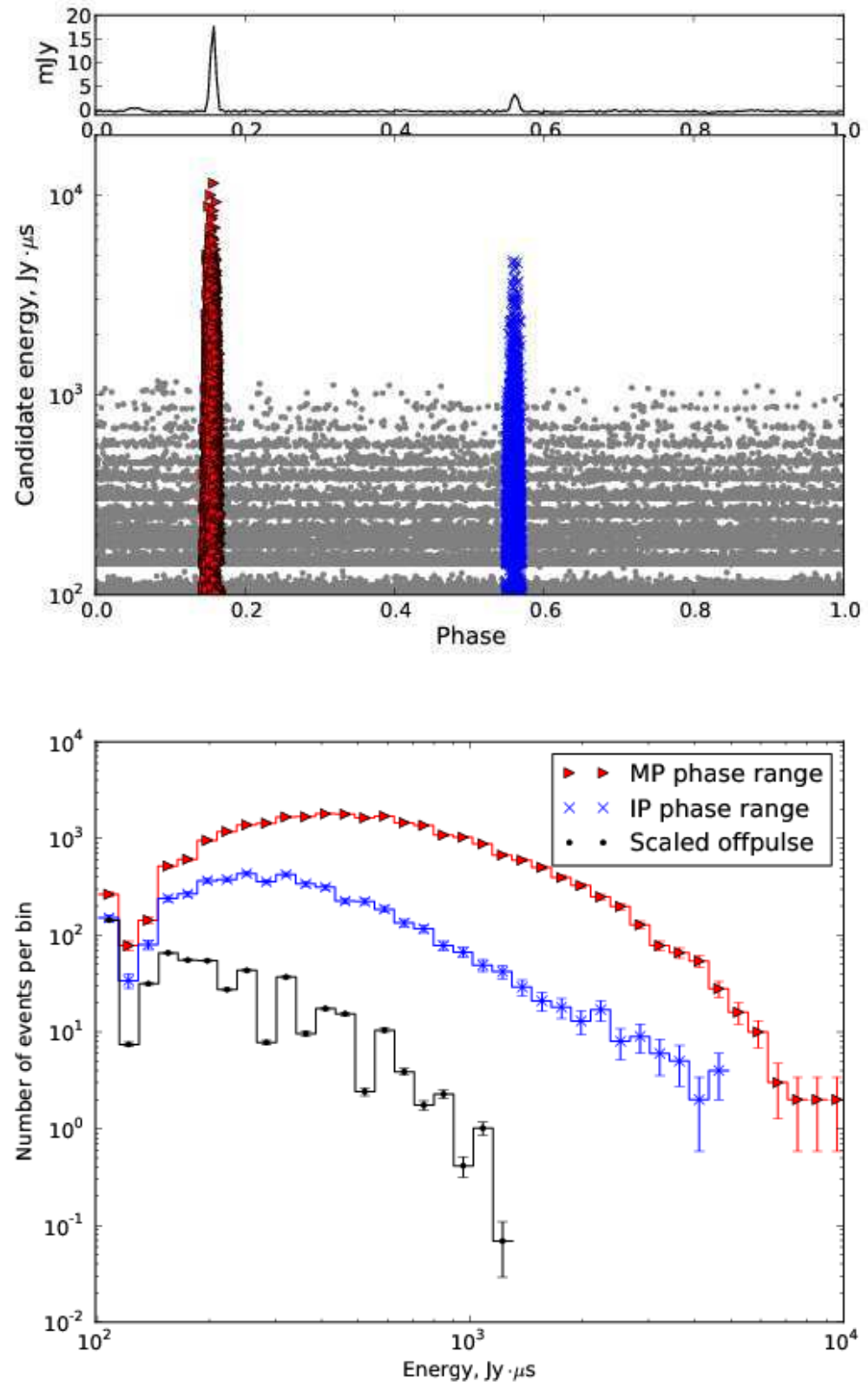

Fig. 3.2. - Top: GP candidates, selected by matched-filtering with a set of boxcars of different width. Most of the candidates come from phase regions coincident with the MP (red triangles) and IP (blue crosses). Events outside the MP/IP (grey dots) tend to concentrate at a set of levels because of the width-dependent selection thresholds (see text for explanation). The average profile from the same observation is plotted on top for reference. Bottom: Energy distributions of selected GPs and spurious detections. The energy distribution of spurious detections was scaled by 0.023 to match the size of MP/IP phase windows. The shape of the distributions below $1011 \mathrm{Jy} \cdot \mu \mathrm{s}$ is affected by width-dependent selection thresholds. 
deviation of $0.001 \mathrm{pc} \mathrm{cm}^{-3}$. An example of a giant pulse dedispersed with the corrected dispersion measure is shown in Fig. 3.1 (right).

After correcting the dispersion measure, we dedispersed the raw data using the PRESTO package ${ }^{2}$. For the correlation study, we used only the total intensity time series. More detailed investigation of the recorded GPs, including spectra, polarization, width and energy distribution will be presented elsewhere.

To search for GPs we convolved the dedispersed time series with a series of boxcar functions of varying boxcar width. We used singlepulse_search.py from PRESTO, which accounts for all the possible "phase" offsets of each boxcar and records all events with $\mathrm{S} / \mathrm{N}>5$ on the averaged signal. Such definition of the selection threshold facilitates sifting the duplicate candidates (the ones above the threshold for different boxcar widths, but with the same time of arrival). While comparing different boxcar widths, the peak $\mathrm{S} / \mathrm{N}$ of the averaged signal has a clear maximum when the boxcar width matches the pulse width. However, selecting a candidate by its $\mathrm{S} / \mathrm{N}$ on the averaged signal effectively results in a width-dependent energy threshold. After averaging by $n$ samples, the standard deviation of the signal drops by $\sqrt{n}$. Therefore $\mathrm{S} / \mathrm{N}$ of 5 on the averaged signal corresponds to a mean $\mathrm{S} / \mathrm{N}$ of $5 / \sqrt{n}$ on the signal with original time resolution. The energy of each candidate was calculated by multiplying the mean $\mathrm{S} / \mathrm{N}$ by the corresponding boxcar width. Thus, the minimum detectable energy of the $n$-sample wide candidate was $5 \sqrt{n} \cdot \mathrm{SEFD} \cdot t_{\mathrm{res}}$, or $101.1 \sqrt{n} \mathrm{Jy} \cdot \mu \mathrm{s}$ with SEFD of 7.9 Jy and time resolution of $2.56 \mu \mathrm{s}$. In our analysis we used boxcars of 1, 2, $3,4,6,9,14,20,30,45,70$ and 100 samples and the width-dependent energy thresholds can be seen on Fig. 3.2 (left) as the concentration of events at the energy levels proportional to the square root of the boxcar widths used.

Most of GP candidates were detected within two narrow phase regions, each spanning 0.022 pulsar rotations and coincident with the main pulse and the interpulse on the average profile, obtained by folding the dedispersed time series with the pulsar period (see Fig. 3.2, left). Outside the MP/IP region the phase

\footnotetext{
${ }^{2}$ http://www.cv.nrao.edu/ sransom/presto/
} 
distribution of events is flat, as it should be for noise. No excess of events was found in the Low Frequency Component (peaks at the phase 0.05).

As has been reported by Popov \& Stappers (2007), giant pulses from the MP and IP have different energy distributions: IP GPs are well fitted by a single power-law, whereas MP GPs obey a broken power-law, with the energy of the break depending on observing frequency as $E_{\mathrm{break}}=7(\nu / \mathrm{GHz})^{-3.4} \mathrm{kJy} \cdot \mu \mathrm{s}$. For our observing frequencies, $E_{\text {break }}$ ranges from 0.8 to $5.0 \mathrm{kJy} \cdot \mu \mathrm{s}$, making the distribution of MP GPs detected in the $800 \mathrm{MHz}$ band a convolution of several different broken power-laws.

As can be seen from Fig. 3.2 (right), above $300 \mathrm{Jy} \cdot \mu$ s the distributions for events in MP and IP windows behave as predicted. We believe that the roll-off below $300 \mathrm{Jy} \cdot \mu \mathrm{s}$ is most probably due to the underestimation of width (and thus energy) for faint pulses. Also, all distributions are affected by width-dependent selection thresholds. This impact is best visible as a dip between 101.1 Jy· $\mu$ s and $101.1 \sqrt{2} \mathrm{Jy} \cdot \mu \mathrm{s}$. In any case, the lowest energy of events is 20 (for MP window) or 100 (for IP) times bigger than the corresponding energy of the mean profile. By the formal definition of GPs as those with energy exceeding 10 times the energy of the mean profile (Cairns 2004), even the faintest pulses in our sample can be considered giant. Thus, for the subsequent analysis we kept all events in the on-pulse phase range, 25986 in MP and 4636 in IP. In the off-pulse phase range, which spanned 0.956 pulsar rotations, 23366 events were detected. Thus, we estimate the fraction of false GPs to be about $2.1 \%$ in the MP window and $11.6 \%$ in the IP window.

We put the list of GP arrival times in TEMPO2 format (Hobbs et al. 2006) and converted to the barycentric reference frame for the subsequent correlation analysis with Chandra data. We used the TDB time system and DE405 solar system ephemeris. Times of arrival (TOAs) were corrected for delay due to propagation in the ionized interstellar medium (ISM). Timing errors due to errors in DM are up to $1.8 \mu \mathrm{s}$.

The observed rate and peak flux densities of GPs are inevitably affected by 

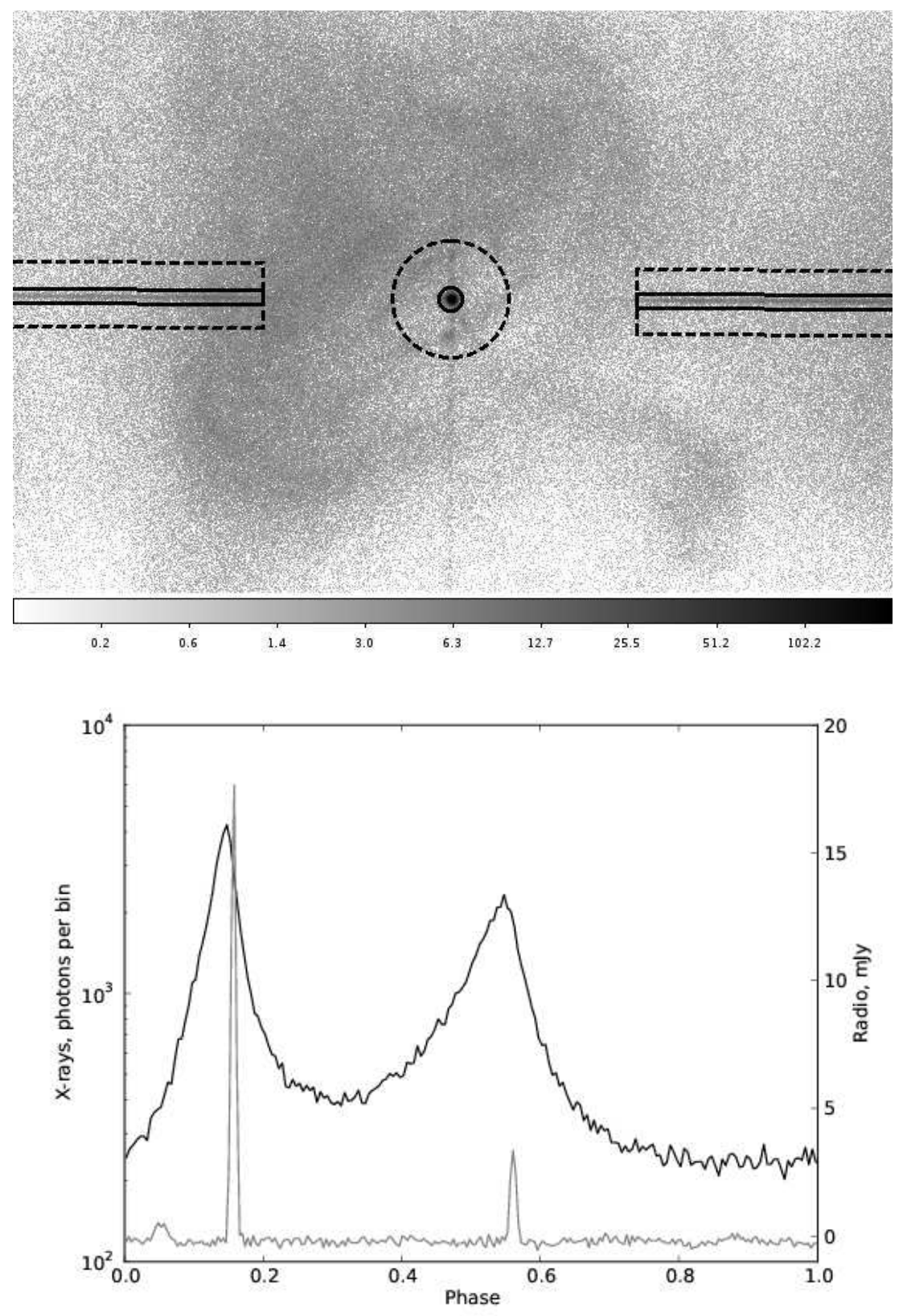

Fig. 3.3.- Top: central part of the HRC-S image. Solid line: source region, zero order (circle) and dispersed spectra (boxes, only central part shown). Dashed line: background region. Due to an imperfect PSF model, we still detect weak pulsed emission in the background, so we used only photons within pulsar phases $0.7-1.0$. Bottom: phase distribution of the extracted X-ray photons (black). The mean profile of the radio emission is overplotted for reference (grey). 
scintillation in the ISM and the Crab Nebula. To find the scintillation timescales we constructed the autocorrelation function for the GP rate. We did not find any GP rate change on any timescales down to $1 \mathrm{~s}$, the smallest bin size possible with our limited sample of GPs. Also, we found no scintillation patterns in the two-dimensional (observing frequency and time) autocorrelation function for the dedispersed data (Rickett 1977), averaged over 5 seconds to increase the signal to noise ratio.

By fitting an exponential scattering tail to the average profile of the strongest pulses $(\mathrm{S} / \mathrm{N}>40)$, we estimated the scattering time to be about $10.3 \mu \mathrm{s}$, which is about 10 times bigger than the expected broadening by interstellar scattering (Kuzmin et al. 2002) and could be explained by scintillations of the nebula or by the intrinsic duration of GPs (Crossley et al. 2010). In any case, both expected and measured scattering times give a decorrelation bandwidth at least an order of magnitude smaller than the width of one frequency channel. The refractive interstellar scintillation (RISS) timescale, $\tau_{\text {RISS }}$, can be scaled using the $\nu^{-2.2}$ dependence derived from a five frequency dataset (Rickett \& Lyne 1990). For $1.5 \mathrm{GHz}$ it corresponds to $\tau_{\mathrm{RISS}}=1.2$ days, much bigger than our observing timespan. The diffractive interstellar timescale is $\tau_{\text {DISS }}=\tau_{\text {RISS }}$. $\triangle f_{\text {DISS }} / f=1 \mathrm{~s}$. However, we did not have enough GPs or sensitivity in folded data series to detect scintillations on such short timescales.

\subsection{X-ray observations}

The X-ray observations were carried out with Chandra, using the Low-Energy Transmission Grating (LETG) and High-Resolution Camera spectroscopy detector (HRC-S) in timing mode (ObsId 11245). To ensure good timing accuracy we used a specific HRC set-up that selected only signals from the central HRC$\mathrm{S}$ segment, disabled all on-board vetoing, and set the trigger-level $=50$. The errors in photon arrival times in timing mode are about $16 \mu \mathrm{s}^{3}$. Simultaneous

\footnotetext{
${ }^{3}$ http://asc.harvard.edu/cal/Hrc/
} 
observing time with the GBT comprised 5.4 hours.

We created the level=2 photon lists using the data from the Chandra X-ray Center pipeline ${ }^{4}$, and barycentered the arrival times with axbary from the CIAO software package. Axbary converts all arrival times to the TDB time system, so we could assign each photon its pulse phase using TEMPO2 with the same pulsar and solar system ephemeris as for the radio data.

While checking the X-ray data, we noticed that the count flux from any region on the detector gradually grows by about $20 \%$ over the course of observations. Specifically, if $f(t)$ is the count flux in some region at a certain moment, then $f(t)$ can be expressed as $k(t) \cdot \overline{f(t)}$, where $\overline{f(t)}$ is the average count flux in that region and $k(t)$ has roughly the same shape for all regions. Interestingly, the phase-resolved flux exhibits the same behavior - the increase of flux in each phase bin is proportional to the mean value of flux in that phase bin. Such a variability pattern cannot be explained by increasing background, since its change would be independent from the pulsar phase. Discussions with the HRC instrument engineers and scientists did not result in an explanation for the rate change (CXC Help Desk Ticket \#13509). The detector particle background appeared to be essentially flat throughout the observation. The only change in instrument conditions seen was a slight temperature change. However, we have no mechanism to explain how it could change the X-ray rate but not the charged-particle rate (Michael Juda, private communication).

Since X-ray flux changes gradually and GP rate is constant over the course of observations, we can still constrain the change in X-ray flux around GPs, by comparing the X-ray flux around GPs to the X-ray flux around random time points with the same pulsar spin phase range as for the GPs. However, measuring the absolute value of the flux is currently not possible.

For the subsequent analysis we extracted photons from $1.9^{\prime \prime}$ radius circle around the pulsar image in the 0th order LETG spectrum and from the two boxes around higher spectral orders (see Fig. 3.3, left, solid line). We did not make

\footnotetext{
${ }^{4}$ http://cxc.harvard.edu/ciao/guides/hrc_data.html
} 

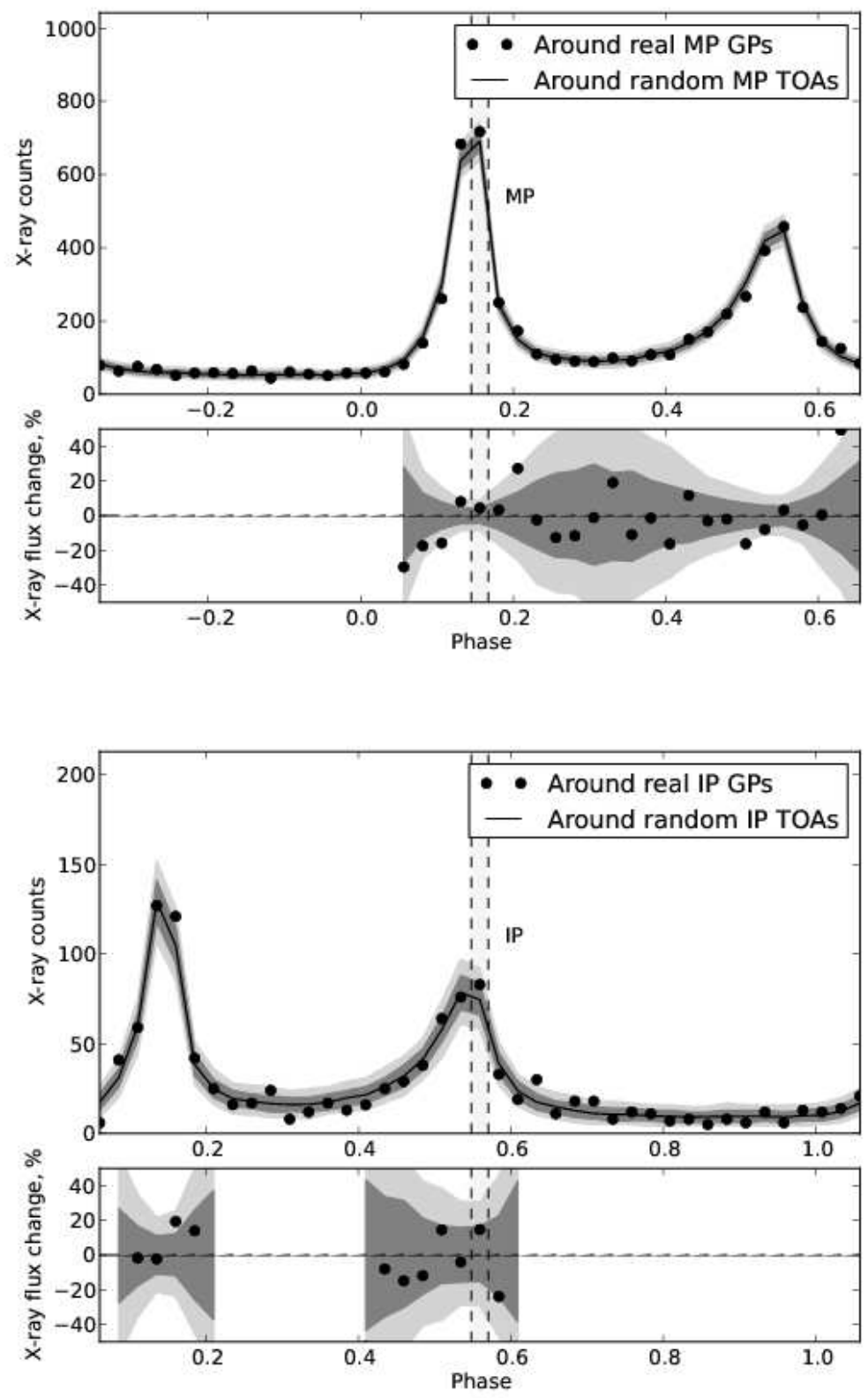

Fig. 3.4.- Upper subplots: X-ray counts in the radio emission region around MP GPs (left, within dashed lines) and IP GPs (right, within dashed lines) together with $\mathrm{X}$-ray counts in the windows of similar size, shifted by the size of the window within one pulsar period from a GP. Black line: X-ray counts around the same number of MP/IP GPs with randomized integer part of phase. Shaded regions $-68 \%$ and $95 \%$ percentile on the pool of simulated datasets. Bottom subplots: relative change of X-ray flux $\left(f_{\mathrm{GP}}-f_{\text {noGP }}\right) / f_{\text {noGP. }}$. Only regions with good statistics are shown. 
any cuts by energy, but most photons in our sample had energies of $1.5-4.5 \mathrm{keV}$. Figure 3.3, right shows the phase distribution for our X-ray sample, with the radio profile overplotted for reference. Giant pulses (which coincide with the peak of radio emission) are delayed with respect to the maximum of the X-ray profile by about 0.01 of pulsar phase. This confirms the result reported by Rots et al. (2004).

The average count rate on the whole detector was about $100 \mathrm{cts} / \mathrm{s}$, which is well below telemetry saturation limit of $184 \mathrm{cts} / \mathrm{s}$. The average dead time fraction was 0.9897 , so the dead time after each recorded photon comprised $100 \mu \mathrm{s}$. This time is bigger than the duration of a typical GP and only a few times smaller than the size of MP/IP emission window. However, since the average number of X-ray photons per one pulsar period in our observations is quite low - only 3 photons from the whole detector and 0.2 photons from our region of interest, the dead time will not considerably affect the correlation results on any time scale, unless the correlated photons come in bunches of less than $100 \mu \mathrm{s}$.

\subsection{Correlation Analysis}

Ideally, for measuring the change in X-ray flux in the vicinity of GPs one should identify all GPs in the data sample and compare the X-ray flux in a selected window close to GPs $\left(f_{\mathrm{GP}}\right)$ to the X-ray flux in an identical phase window close to the periods without GPs $\left(f_{\text {noGP }}\right)$.

In practice, our GP samples always contain some fraction of spurious detections $(s)$. We do not know the true fraction of GPs in the sample $(t)$ and there is background X-ray flux $\left(f_{\mathrm{bkg}}\right)$.

Thus, if we measure the X-ray flux in some phase window close to GPs, the measured flux will be related to the true flux $f_{\mathrm{GP}}$ as follows (assuming that $s$ and $t$ are small): 

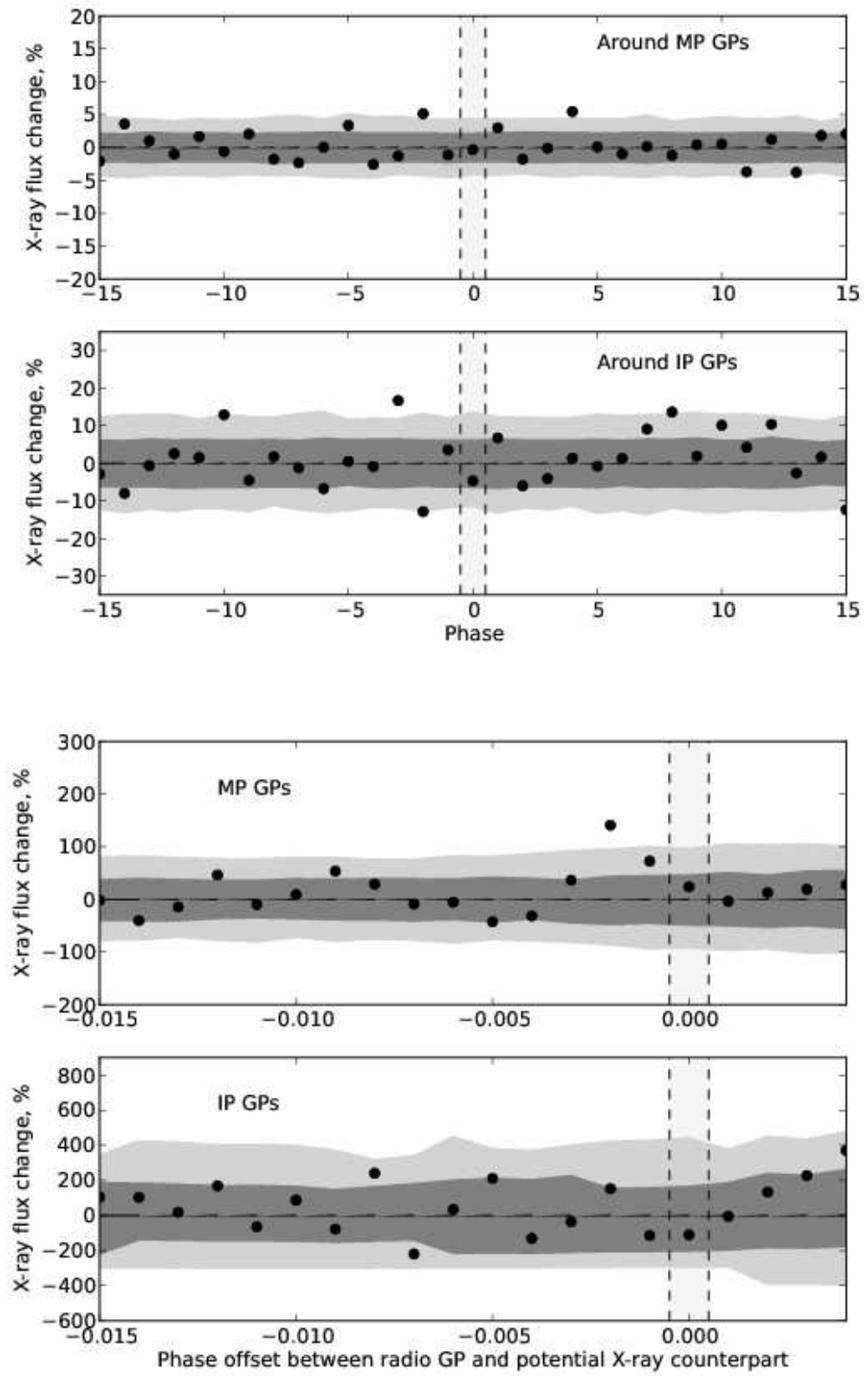

Fig. 3.5.- Left: Relative change of X-ray flux $\left(f_{\mathrm{GP}}-f_{\text {noGP }}\right) / f_{\text {noGP }}$ in a period around a GP (within dashed lines) and in one-period windows shifted by the size of window up to \pm 15 periods around GPs. Black dots: change of the flux around real GPs. Shaded regions $-68 \%$ and $95 \%$ percentile on the pool of GP lists with random arrival times. Right: Relative change of the X-ray flux around a GP on the timescale of the GP duration (within dashed lines) and on the same timescale but with phase lags from -0.015 to 0.004 . For the average emission profile, the peak of the X-ray main pulse is shifted with respect to the peak of average radio pulse by -0.01 . 


$$
f_{\mathrm{GP}}^{\mathrm{meas}}=f_{\mathrm{bkg}}+(1-s) f_{\mathrm{GP}}+s f_{\text {noGP }}
$$

At the same time, by counting photons in the same window close to the randomly selected periods we can estimate the average (over all periods) X-ray flux in our correlation window:

$$
f_{\mathrm{aver}}^{\text {meas }}=f_{\mathrm{bkg}}+t f_{\mathrm{GP}}+(1-t) f_{\mathrm{noGP}} .
$$

Then, $f_{\mathrm{GP}}$ and $f_{\text {noGP }}$ can be found by solving both equations simultaneously.

We performed this procedure separately for MP and IP GPs and for the three different timescales - GP duration, radio emission phase window and pulsar period. For every timescale we used not only the correlation window centered on GPs, but also a set of windows of the same size, shifted with respect to GPs. Background flux $f_{\mathrm{bkg}}$ was measured in the narrow region around the initial extraction region (Fig. 3.3, left, dashed line). Due to imperfect PSF modeling, we still detected weak pulsed emission in the background, so we used only photons with pulse phases 0.7-1.0. The fraction of false GPs $s$ was calculated as in Sec. 3.2. For the fraction of periods with GPs, we adopted the fraction of periods with detected GPs, 0.04 for MP GPs and 0.008 for IP GPs, although the true fraction remains unknown. However, the limits on flux change are not sensitive to the change of $t$, as long as it remains small. For the two larger correlation timescales, the radio emission phase window and the pulsar period, varying $t$ up to 0.2 does not increase the estimated X-ray flux change in vicinity of GP by more than a factor of two.

For all correlation windows, for each GP we selected all X-ray photons within the given window (photons that satisfied the criterion for multiple GPs were counted multiple times). For comparison, we also measured $f_{\text {aver }}^{\text {meas }}$ in the identical windows by creating 1000 lists of fake GPs which had the same phase of arrival as real GPs, but random arrival period. We used simulated lists instead of averaging directly over all periods in order to get the errors on the X-ray flux 
estimates due to the limited number of photons/GPs. The histograms of photons around real GPs were compared with mean, $68 \%$ and $95 \%$ percentiles on the pool of simulated datasets and the relative flux change, $\left(f_{\mathrm{GP}}-f_{\text {noGP }}\right) / f_{\text {noGP }}$ was calculated with equations 3.1 and 3.2.

For all unshifted correlation windows the value of the X-ray flux stayed within the corresponding $95 \%$ percentile measured on the pool of simulated GP lists with random arrival period. In other words, the measured change of the X-ray flux in a window around a GP was within $2-\sigma$ intrinsic scatter of this value due to limited number of GPs/photons. In a few shifted windows the X-ray flux exceeded 2- $\sigma$ level, but it always stayed within $3 \sigma$.

\subsubsection{Correlation on the timescale of radio emission win- dow}

Figure 3.4 (top part of each subplot) shows the X-ray counts in the radio emission region around MP GPs (left, within dashed lines) and IP GPs (right, within dashed lines) together with X-ray counts in the windows of same size, shifted by the size of the window within one pulsar period from a GP. The bottom part of each subplot shows the relative flux change, $\left(f_{\mathrm{GP}}-f_{\text {noGP }}\right) / f_{\text {noGP }}$. The $\mathrm{X}$-ray flux in MP/IP emission regions during periods with GPs stays within $2 \sigma$ intrinsic scatter of this value due to limited number of GPs/photons, which is $\pm 10 \%$ for $\mathrm{MP}$ and $\pm 30 \%$ for IP.

\subsubsection{Correlation on the timescale of pulsar period}

If the process that generates GPs has somewhat less prominent long-term influence on X-ray flux, it would be easier to detect it with larger correlation windows. Figure 3.5 (left) shows the relative X-ray flux change for a timescale of one pulsar period around GPs (within dashed lines) and in one-period windows shifted by the size of window up to \pm 15 pulsar periods around GPs. The 
X-ray flux in the same period as GPs stays within 2- $\sigma$ intrinsic scatter of this value due to limited number of GPs/photons, which is $\pm 5 \%$ for MP and $\pm 10 \%$ for IP.

\subsubsection{Correlation on the timescale of GP duration}

If X-ray photons are emitted simultaneously with GPs, then the change of X-ray flux will reveal itself on timescales comparable to the widths of GP. However, if the paths of X-rays and radio emission are different throughout the magnetosphere, then the X-ray "pulses" will be shifted with respect to the radio GPs. Since the radio peak is delayed in phase from the X-ray one by 0.01 , we looked for the correlation on the timescale of GPs with 20 phase offsets starting from -0.015 with a step size of 0.001 .

Most of our GPs have widths less than the X-ray photon timing precision $(16 \mu \mathrm{s})$, so only rough estimates of the change of X-ray flux was possible. We looked for the correlation in a window around each GP with the size of the window equal to the width of that GP plus $32 \mu \mathrm{s}$. For such choice of correlation window $s$ and $t$ depend in a complex way on the distribution of widths of the GPs. However, they bring only minor corrections, so we set both of them to 0 . The change of the X-ray flux $\left(f_{\mathrm{GP}}-f_{\text {noGP }}\right) / f_{\text {noGP }}$ was estimated from the following system of equations:

$$
\begin{gathered}
f_{\mathrm{GP}}^{\text {meas }} \approx f_{\mathrm{bkg}}+\frac{f_{\mathrm{GP}} \cdot w_{\mathrm{GP}}}{32 \mu \mathrm{s}+w_{\mathrm{GP}}}+\frac{f_{\mathrm{noGP}} \cdot 32 \mu \mathrm{s}}{32 \mu \mathrm{s}+w_{\mathrm{GP}}} . \\
f_{\mathrm{aver}}^{\text {meas }} \approx f_{\mathrm{bkg}}+f_{\mathrm{noGP}} .
\end{gathered}
$$

From Fig. 3.5 (right) shows that the relative X-ray flux on a timescale of the GP duration stays within $\pm 3 \sigma$ for all phase lags chosen. The X-ray flux during GPs (zero phase lag) is within $\pm 2 \sigma$ and does not increase more than 2 times for MP GPs and 5 times for IP GPs. 


\subsection{Discussion}

The lack of significant change of X-ray flux during giant pulses should be accounted for by any theory of GP generation. Currently, there exist a number of giant pulse theories, which place GPs in different parts of the magnetosphere and draw in various emission mechanisms. Unfortunately, none of the theories that we are aware of quantitatively describe possible X-ray byproducts of radio GPs. Thus, below we will give only some qualitative considerations.

Gil \& Melikidze (2004) argue that GPs are due to coherent curvature emission of relativistic charged solitons, which fly along magnetic field lines in the polar region deep inside the light cylinder. The lack of strong synchrotron X-ray emission accompanying radio GPs suggests that the most particles in solitons have zero pitch angle with respect to magnetic field.

The model of Weatherall (1998) explains GPs using spatial collapse of wave packets in strongly turbulent polar cap plasma. This model describes direct conversion of plasma wave turbulence into radio emission, and since it does not imply a significant increase of particle density in the emitting volume, little correlation with high-energy incoherent photons is expected.

Harding et al. (2008) directly predicts time and phase correlation of photons below $200 \mathrm{MeV}$ with radio pulses. Radio photons undergo resonant absorption by the secondary electron-positron plasma on the field lines interior to the slot gap. Because of this, charged particles are able to maintain significant pitch angles, and emit synchrotron X-ray photons. However, quantitative limits on the energy of the photons and amount of X-ray flux change increase are not given.

Our results rule out strong correlation between radio GPs at $1.1-1.9 \mathrm{GHz}$ and X-ray photons of $1.5-4.5 \mathrm{keV}$. However, there can still exist weak correlation on a few percent level, similar to those observed in the optical band (Shearer et al. 2003) and/or correlation between GPs at some particular radio wavelength and specific high-energy band. 
Chapter 4

\section{B1821-24A (M28A): Average Profile and Single Pulses}




\subsection{Introduction}

B1821-24A is an isolated 3.05-millisecond pulsar in the globular cluster Messier 28. The pulsar has an uncommonly large period derivative, $\dot{P}=1.62 \times 10^{-18}$ (Verbiest et al. 2009), about two orders of magnitude larger than typical $\dot{P}$ for millisecond pulsars (MSPs). The large observed $\dot{P}$ is likely to be an adequate estimate of pulsar's magnetic braking: proper motion and acceleration in the Galactic gravitational potential contribute negligibly and any considerable influence by acceleration in the gravitational potential of M28 is unlikely (Foster et al. 1988).

The combination of large $\dot{P}$ and small spin period makes this pulsar the most energetic MSP known, with spin-down luminosity $\dot{E}=2.2 \times 10^{36} \mathrm{erg} / \mathrm{s}$. B1821-24A is also one of the most luminous MSPs in X-rays. Unlike the majority of MSPs, which show broad thermal pulsations in their X-ray profiles, it shows two sharp peaks of highly beamed non-thermal radiation (Bogdanov et al. 2011).

Because of its large derived magnetic field and rotational energy loss rate $\dot{E}$, B1821-24A is a potential $\gamma$-ray source. However, the pulsar was detected only with $4.2 \sigma$ significance by AGILE (Pellizzoni et al. 2009) in the $100 \mathrm{MeV}-30 \mathrm{GeV}$ energy range during one 6 -day subset of their observations. The broad $\gamma$-ray peak was reported to coincide with the peak of radio emission at $1.4 \mathrm{GHz}$. The pulsar has not been detected by Fermi (S. Ransom, private communication). In radio, $\mathrm{B} 1821-24 \mathrm{~A}$ has a complex profile with multiple components, some of which are almost completely linearly polarized. It exhibits strong profile evolution as a function of frequency, at least in the GHz range (Ord et al. 2004, and references therein).

In 2001, Romani \& Johnston detected individual pulses from B1821-24A with energies exceeding the mean pulse energy by up to 50 times. These bright pulses come in narrow phase windows on the trailing edges of two average profile components and coincide with the peaks of integrated X-ray emission (Romani \& Johnston 2001). The properties of the individual pulses resemble 
those of giant pulses (GPs): ns $-\mu$ s pulses with power-law energy distribution and high degree of polarization (Knight et al. 2006).

In this paper we present results from the first wide-band study of B1821-24A, covering frequencies from 720 to $920 \mathrm{MHz}$ and from 1100 to $2400 \mathrm{MHz}$. We examine the frequency dependence of phase-resolved average radio flux, the polarization of the average profile, and describe the individual pulses.

\subsection{Observations and calibration}

We observed B1821-24A with the $100 \mathrm{~m}$ Green Bank Telescope for 27.5 hours during 12 epochs in 2010-2012. Observations were conducted in search mode with the GUPPI pulsar backend ${ }^{1}$ at 820, 1500 (L-band) or 2000 (S-band) MHz. Observing bandwidths were $200 \mathrm{MHz}$ at $820 \mathrm{MHz}$ and $800 \mathrm{MHz}$ in L and Sbands. Three of the observing sessions in L and S-bands in 2010 were done in incoherent dedispersion mode, with the pulsar signal integrated in each of 2048 frequency channels. During other sessions the pulsar was observed with 512 frequency channels and the incoming signal was coherently real-time dedispersed within each channel with the average dispersion measure for the pulsars in the globular cluster $\left(120 \mathrm{pc} \mathrm{cm}^{-3}\right.$; Stairs et al. in prep.). The sampling interval for incoherent modes was $64 \mu \mathrm{s}$, while for the coherent modes was $10.24 \mu \mathrm{s}$.

Following standard timing procedure, we obtained times of arrivals (TOAs) by correlating pulse profile templates with the subintegrations of the data folded modulo the predicted pulsar topocentric period. Since the pulsar profile changes substantially with our observing bands, different pulse profile templates were used for each band. At first, we integrated over the whole band, producing one TOA per minute at the central frequency of the band. Using TEMPO ${ }^{2}$, we refined $P, \dot{P}$ and the pulsar's astrometric coordinates. To measure the dispersion measure (DM) we re-folded the data with updated ephemerides and

\footnotetext{
${ }^{1}$ https://safe.nrao.edu/wiki/bin/view/CICADA/NGNPP

${ }^{2}$ http://www.atnf.csiro.au/people/pulsar/tempo/
} 
obtained 16 TOAs for each observing session - one TOA per half of the observing duration and for each $1 / 8$ of the frequency band. DM was fitted with TEMPO separately for each observing session. Fig. 4.1 shows DM versus time. After updating the DM, we rotated all folded archives so that the peak of component P2 in the average profile (see Knight et al. (2006) for details) was at phase 0.5. Prior to each observation we recorded a pulsed calibration signal, which was used together with standard flux calibrators (B1442+101 for L-band, 3C190 for S- and $820 \mathrm{MHz}$ ) to correct for the instrumental response of the receiver system. Polarization calibration for L-band and $820 \mathrm{MHz}$ was conducted using pre-determined Mueller matrix solutions, which described the cross-coupling between orthogonal polarizations in the receivers (van Straten 2004). The Mueller matrix was determined using pcm from observations of PSR B0450+55 in Lband and PSR B1744-21A at $820 \mathrm{MHz}$. For S-band polarization calibration we assumed that the feed is ideal and composed of two orthogonally polarized receptors.

Fig. 4.2 (top) shows the unwrapped position angle, $\mathrm{PA}=0.5 \tan ^{-1}(U / Q)$ for the peak phase bins of the two brightest average profile components versus the square of observing wavelength $\lambda^{2}$. For all three bands and all observing sessions PA depends linearly on $\lambda^{2}$, as expected for Faraday rotation:

$$
\mathrm{PA}=\mathrm{PA}_{0}+\lambda^{2} \mathrm{RM}
$$

where rotation measure (RM) is proportional to the average magnetic field strength along the line of sight weighted by the local electron density. The observed rotation measure is a sum of interstellar $\mathrm{RM}_{\mathrm{IS}}$ and a contribution from Earth's ionosphere $\mathrm{RM}_{\text {iono. }}$. The latter depends on the zenith angle of the pulsar and it's angular distance from Sun at the moment of observation. To estimate the $\mathrm{RM}_{\text {iono }}$ we used ionFR software (Sotomayor-Beltran et al. in prep.), which uses the last release of the International Geomagnetic Reference Field (IGRF11) and global ionospheric maps to predict the $\mathrm{RM}_{\text {iono }}$ along a given line of sight at a specific geographic location. For our set of parameters $\mathrm{RM}_{\text {iono }}$ 
varied from 1 to $6 \mathrm{rad} \cdot \mathrm{m}^{-2}$ and changes by $0.2-1.5 \mathrm{rad} \cdot \mathrm{m}^{-2}$ during an observing session. Fig. 4.2 (bottom) shows the observed RM and RM $\mathrm{IS}_{\mathrm{IS}}$ versus the angular distance between B1821-24A and the Sun. The observed RM was found by fitting ${ }^{3}$ Eq. 4.1 to Fig. 4.2 (top). The errors on $\mathrm{RM}_{\mathrm{IS}}$ are uncertainties in $\mathrm{RM}_{\mathrm{obs}}$ and $\mathrm{RM}_{\text {iono }}$ added in quadrature.

Over the set of twelve observing sessions the average $\mathrm{RM}_{\mathrm{IS}}$ was $81 \mathrm{rad} \cdot \mathrm{m}^{-2}$, with standard deviation of $1 \mathrm{rad} \cdot \mathrm{m}^{-2}$. This result is somewhat higher than $77.8 \pm 0.6 \mathrm{rad} \cdot \mathrm{m}^{-2}$, published by Yan et al. (2011b).

The inhomogeneities in the interstellar medium (ISM) distort the radio wave front, broadening observed pulses and causing correlated variations of the pulsar flux in frequency and time. After traveling through the ISM, sharp pulses emitted by a pulsar will be measured on Earth as (approximately) convolved with a one-sided exponential function with a scattering time scale $\tau_{\mathrm{sc}}$. Giant pulses (see Sect. 4.4) are perfect tools for measuring $\tau_{\mathrm{sc}}$, because of their large peak flux density and short duration. For each session we divided the observing band into 4 sub-bands and obtained average GP profiles in each sub-band by aligning all GPs detected on that day at their rising half-maximum-intensity point. The exponential function was fitted to the scattering tail at every frequency where the tail was visible, namely $720-1300 \mathrm{MHz}$. At $1300 \mathrm{MHz}$, where we had 7 observations, the scattering time varied from 1 to 3 time samples (SMR put that in time units) from day to day. Variable scattering timescales for B1821-24A were previously observed by Knight et al. (2006). Given the paucity and scatter of data, we did not attempt to fit for a frequency dependence of $\tau_{\mathrm{sc}}$, adopting $\tau_{\mathrm{sc}} \sim f^{-4.4}$ for a Kolmogorov spectrum of turbulence. Fitting this dependence to measured $\tau_{\mathrm{sc}}$ gives $\tau_{\mathrm{sc}}=15 \mu \mathrm{s}$ at $1 \mathrm{GHz}$.

\footnotetext{
${ }^{3}$ This fit and all other fits in this work was performed with publicly available Markov-Chain Monte Carlo software called PyMC (http://pypi.python.org/pypi/pymc/). In certain cases, we cross checked our results with standard least-squares techniques. In all cases, we assumed uniform priors on the parameters, which is appropriate for the estimation of so-called 'location' parameters (i.e. not restricted to $\geq 0$, where a Jeffery's prior is normally assumed) and implemented Gaussian likelihoods. Therefore, for the fits of linear parameters, our analysis is analytically equivalent to linear least-squares/maximum-likelihood methods. The errors on fitted parameters represent bounds on $68 \%$ highest probability density region.
} 
The decorrelation bandwidth $\delta f \approx 1 /\left(2 \pi \tau_{\mathrm{sc}}\right)$ varied from $2 \mathrm{kHz}$ at $720 \mathrm{MHz}$ to $600 \mathrm{kHz}$ at $2400 \mathrm{MHz}$. For all bands it is smaller than the width of our frequency channels. The diffractive scintillation timescale ranged from $45 \mathrm{~s}$ at $720 \mathrm{MHz}$ to $3 \mathrm{~min}$ at $2400 \mathrm{MHz}$, assuming the pulsar's transverse velocity to be $110 \mathrm{~km} / \mathrm{s}^{4}$. The refractive scintillation timescale varied from 129 days at $720 \mathrm{MHz}$ to 9 days at $2400 \mathrm{MHz}$. The modulation strength of pulsar flux in L-band $\left(m=\sigma_{I} / I_{\text {aver }}\right)$ was measured to be 0.15 , which agrees with $m=0.2$ prediction for the refractive scintillation.

\subsection{Average profile}

\subsubsection{Spectral indices}

The pulsar's phase-integrated flux between 720 and $2400 \mathrm{MHz}$ does not show any deviations from a power-law with index of $-2.55 \pm 0.05$. This is in agreement with Foster et al. (1991), who reported $-2.3 \pm 0.2$ over a much wider frequency range of $30-1700 \mathrm{MHz}$.

Fig. 4.3 shows the average pulse profile of B1821-24A at 820, 1500 and $2000 \mathrm{MHz}$. Following Knight et al. (2006) we designate the components at phases 0.2, 0.5 and 0.7 as P1, P2 and P3. The relative heights of profile peaks at a given frequency were the same for all observing sessions, unlike reported by Backer \& Sallmen (1997). Weak circular polarization in components P1 and P2 in Lband has the same amplitude as observed by Yan et al. (2011a) at $1369 \mathrm{MHz}$. It should be noted that in this work we use different sign convention for circular polarization (van Straten et al. 2010), so our positive V corresponds to the negative one in Yan et al. (2011a).

Fitting power-laws to phase-resolved flux revealed some systematic residuals (see Fig. 4.4). Between different observing sessions, the deviations from powerlaw had similar shape within each band, but varying amplitude. They can

\footnotetext{
${ }^{4}$ http://www.atnf.csiro.au/people/pulsar/psrcat/
} 
be explained by a combination of an imperfect flux calibration and mixing of Stokes components due to imperfect Mueller matrix. For all observing sessions the deviations from a power-law for any Stokes component in any on-pulse phase bin were not bigger than $15 \%$ of total intensity at that phase and frequency.

Fig. 4.5 shows phase-resolved power-law indices $\alpha$ for the three observing bands. The $\alpha(\phi)$ curves have similar shape for all bands. However, for components P1, P3 and the bright part of P2 $\alpha$ at a given phase $\phi$ tends to have larger negative values with increasing frequency. Phase-resolved spectra are the most steep at the trailing edge of component $\mathrm{P} 1$, reaching $\alpha=-8$ in the phase window of GP arrival in L-band. GPs are also observed at the trailing edge of P3. At $820 \mathrm{MHz}$ the power-law indices in both phase windows of GP arrival are about -4 . At higher frequencies the low signal to noise ratio of the trailing edge of component P3 did not allow us to measure $\alpha$ in the second window of GP generation. Interestingly, the trailing edge of component P2 also exhibits a steepening of the spectra, although somewhat less prominent than P1.

However, it must be noted that scattering in the ISM can contribute to the observed steepening of spectra at the trailing edge of the components in phase windows of about $\tau_{\mathrm{sc}} / P$, since the observed flux at a given phase $\phi_{0}, I_{\mathrm{obs}}\left(\phi_{0}, f\right)$ is actually a convolution of the intrinsic $I_{\text {intr }}(\phi, f)$ with $\exp \left(-\left(\phi_{0}-\phi\right) P / \tau_{\mathrm{sc}}\right)$, where $\tau_{\mathrm{sc}} \sim f^{-4.4}$. For our observations, the scattering time is as big as 0.02 of a pulse or 6 time samples at $720 \mathrm{MHz}$ and less than 1 time sample above $1500 \mathrm{MHz}$.

Also, the rapid evolution of $\alpha(\phi)$ at the very edges (both trailing and leading) of a component can have purely geometrical interpretation, reflecting changes in size of the radio beam with frequency.

\subsubsection{Polarization}

Adopting the idea that pulsar radiation is polarized in the plane of curvature of magnetic field lines, constraints on the configuration of magnetic field and 
emitting regions in the magnetosphere can be placed by examining Stokes components of the average profile. The rotating vector model (RVM), first suggested by Radhakrishnan \& Cooke in 1969 in its simplest form predicts an S-shaped swing of PA across a pulse period and is traditionally used to determine $(\alpha, i)-$ the angles between the spin and magnetic axes and between the spin axes and the observer's line of sight.

Backer \& Sallmen (1997) fit the RVM to the PA variation within components $\mathrm{P} 1$ and $\mathrm{P} 2$ at $820 \mathrm{MHz}$ and found $(\alpha, i)$ to be $\left(40^{\circ}, 90^{\circ}\right)$. Stairs et al. (1999) performed a similar study at $610 \mathrm{MHz}$ and found $(\alpha, i)$ to be about $\left(40^{\circ}, 80^{\circ}\right)$. The standard RVM model is known to experience difficulties explaining the PA curves of millisecond pulsars which are predominantly flat or have complicated multicomponent swings inconsistent with a simple dipolar field (Ord et al. 2004). Chung \& Melatos (2011) developed an extension to RVM which uses numerical models of a current-modified dipolar field together with two empirical emission beam models (filled core and hollow cone) to fit the Stokes phase portraits and PA swings for MSPs. They find $(\alpha, i)$ for B1821-24A to be about $\left(60^{\circ}, 60^{\circ}\right)$, although they used quite low signal-to-noise data.

Fig. 4.6 shows an example of the phase portrait of the Stokes components Q and $\mathrm{U}$ for our observations in L-band. Phase portraits for S-band and $820 \mathrm{MHz}$ look similar. Comparing the phase portraits of Q-U, I-Q and I-U to the look-up tables in Chung \& Melatos (2011) we find that they are qualitatively consistent with both hollow cone and filled core models and with $(\alpha, i)$ of $\left(40^{\circ}-50^{\circ}\right.$, $\left.10^{\circ}\right)$ or $\left(90^{\circ}, 60^{\circ}-90^{\circ}\right)$. The range of possible angular values can be narrowed by numerical fits with variable emission heights, degree of polarization and beam shape, all of which goes beyond the scope of this paper. Additionally, it would be interesting to obtain constraints on the magnetospheric geometry independently from the X-ray light curve. 


\subsection{Single pulses}

We searched for strong single pulses in all available raw data (7 sessions in Lband, one in S-band, and one at $820 \mathrm{MHz}$, all taken with GUPPI in coherent dedispersion mode). At $820 \mathrm{MHz}$, the raw data was subbanded shortly after making folded spectra, so the time resolution in this band was reduced by a factor of 4 compared to L- and S-bands.

We dedispersed and folded the raw data with dspsr, setting the subintegration length to pulsar's spin period. Each subintegration consisted of $P / t_{\text {res }}=298$ phase samples for L- and S-bands and 74 samples for $820 \mathrm{MHz}$. Archives were calibrated in the same way as in 4.2 .

To search for the single pulses we summed all frequency channels and convolved the signal in each period with a series of boxcar functions of varying boxcar width. Minimum boxcar width was set to 1 time sample, the maximum corresponded to 0.1 phase range, the typical width of a component of the average profile. We selected all events with $\mathrm{S} / \mathrm{N}>5$ on the averaged signal. Such a definition of the selection threshold facilitates sifting the duplicate candidates (the ones above the threshold for different boxcar widths, but with the same time of arrival). While comparing different boxcar widths, the peak $\mathrm{S} / \mathrm{N}$ of the averaged signal has a clear maximum when the boxcar width matches the pulse width. However, selecting a candidate by its $\mathrm{S} / \mathrm{N}$ on the averaged signal effectively results in a width-dependent energy threshold. After averaging by $n$ samples, the standard deviation of noise drops by $\sqrt{n}$, therefore $\mathrm{S} / \mathrm{N}$ of 5 on the averaged signal corresponds to the mean $\mathrm{S} / \mathrm{N}$ of $5 / \sqrt{n}$ on the signal with original time resolution. The width-dependent energy threshold influence can be seen in Fig. 4.7 as clustering of events at levels corresponding to the mean $\mathrm{S} / \mathrm{N}$ of $5 / \sqrt{n}$.

For each event selected we recorded its phase, peak flux density, width, energy and spectrum. To save disk space, we reduced the number of channels in the recorded spectra, integrating emission into $6.25 \mathrm{MHz}$ subbands. 
Overall, in each observing session the events fell into three distinct categories: 1) bright and narrow pulses coming in 0.012-width phase windows around phases 0.234 and $0.784 ; 2$ ) faint pulses, mostly broad, clustering around phases 0.2 and $0.5 ; 3)$ faint pulses of all widths, scattered uniformly over all period.

We interpret type 3 as spurious pulses, caused by noise or terrestrial interference. The distribution of widths and energies of these pulses varies in shape to a great extent from session to session.

Type 2 pulses are indistinguishable from type 3 except that there is an evident excess of them in the phase windows of components P1 and P2. Their width and energy distributions follow the shape of the same distributions for noise at any given day. Pulses do not have any distinct spectral features. The similarity to noise together with the phase of arrival times coinciding with the two brightest components of the average profile led us to conclude that type 2 pulses belong to the high $\mathrm{S} / \mathrm{N}$ tail of the distribution of the sum of ordinary single pulses from components $\mathrm{P} 1$ or $\mathrm{P} 2$ with much stronger random noise. Indeed, the typical rms of noise averaged in 0.1 phase window is about $10 \bar{E}_{\mathrm{P} 2}$, where $\bar{E}_{\mathrm{P} 2}$ is the average energy of component P2, calculated in 0.1 phase window around phase 0.5. According to Burke-Spolaor et al. (2012), the most common single pulse energy distribution for normal pulsars is the $\log$-normal with $\log (E)$ having $\sigma_{l}=0.11 \bar{E}$, or, equivalently, the energy of a single pulse being spread with $\sigma_{E}=0.26 \bar{E}$. Thus, in our case the spread of the noise distribution is about 50 times bigger than the spread of energy distribution for normal pulses and although the latter might be log-normal, little can be said about intrinsic properties of type 2 pulses. Type 1 pulses are giant pulses, observed previously by Romani \& Johnston (2001); Knight et al. (2006) in the same phase windows. For further analysis we selected pulses in 0.012-width phase windows around phases 0.234 and 0.784 with average $\mathrm{S} / \mathrm{N}>4$ (shaded regions in Fig. 4.7). GPs coming from the trailing edge of component P1 were on average brighter and more numerous than GPs coming from trailing edge of component P3. However, the number of GPs detected was too small to perform a thorough statistical analysis separately for 
P1 and P3 GPs.

All GPs detected had widths of 1-3 time samples, or less than $30 \mu$ s for L and $\mathrm{S}$ band and less than $120 \mu \mathrm{s}$ for $820 \mathrm{MHz}$. Knight et al. (2006) reported GPs at $1300 \mathrm{MHz}$ to have widths between $750 \mathrm{~ns}$ and $6 \mu \mathrm{s}$, well below our time resolution.

The energies of individual giant pulses can be as high as $100 \bar{E}$, where $\bar{E}$ is the energy of the average pulse. Since GPs occupy less than $1 \%$ of pulsar phase, the mean flux during a giant pulse can exceed the average pulsar flux by $10^{4}$ times. The energy distribution of GPs in all three bands is apparently powerlaw with spectral index -1.5 except for pulses with energies less than about $4 \bar{E}$. We attribute the flattening of GP energy distribution at low energies to our selection threshold, which is based on width-averaged signal-to-noise ratio and does not take into account variations of observed pulsar flux due to refractive scintillations or to change in system equivalent flux density as the pulsar approached the horizon.

Giant pulse spectra consist of a series of narrow-band patches (see Fig. 4.10). The decorrelation bandwidth of interstellar scintillation is an order of magnitude smaller than the $6.25 \mathrm{MHz}$ frequency resolution of ou GP spectra. We measured the frequency width of individual patches by applying a "friends-of-friends" algorithm to the width-averaged spectrum of total intensity, $I(f)^{5}$.

For all bands most patches have widths of 1-2 frequency samples, or below $12 \mathrm{MHz}$ (see Fig. 4.9, left). We did not find any obvious dependence of the width of the patch on its central frequency, although the coarse frequency resolution precludes us from any definite conclusions.

Previously, fine spectral features were observed in GPs from the Crab pulsar (Hankins \& Eilek 2007). At frequencies $f>4 \mathrm{GHz}$, GPs from the Interpulse

\footnotetext{
${ }^{5}$ The algorithm consisted of the following: on $I(f)$ find the $f_{0}$ with maximum $I, I_{0}$. From this point, go up and down in frequency until $I$ reaches $a I_{0}$ or the average plus one standard deviation of noise. Record the boundaries of the patch, $f_{0}^{\text {low }}$ and $f_{0}^{\text {high }}$. Substitute $I$ within the patch with zeros, find next maximum $I_{1}$ and so on. Repeat until $I_{n}$ reaches the average plus 3 standard deviations of noise. The results presented here are obtained with $a=0.25$ although they practically do not change with $a$ in the range of $0.15-0.35$
} 
were continuous in time, spreading out over a few $\mu$ s and their spectra consisted of series of proportionally spaced bands, with the width of the band $\delta f$ scaling as $0.06 f$. Surprisingly, GPs from the Main Pulse were composed of $\sim 0.1 \mu \mathrm{s}$ individual narrowband shots with $\delta f / f \sim 0.1$. If viewed with several microsecond time resolution, individual shots merged together, producing broad-band patchy spectra. Such GPs were explained by the strong plasma turbulence (Weatherall 1998), which involves spatial collapse of wave packets in strongly turbulent polar cap plasma. We did not see any evidence of regularly spaced emission bands for our data. However, the bands for Interpulse GPs from the Crab pulsar are known to drift up in frequency across GP. Since our GPs were unresolved, such drift, if exists, would reduce the apparent modulation. Overall, B1821-24A GP spectra resemble more the ones for Main Pulse Crab GPs (Hankins \& Eilek 2007), although we notice that our GPs have more prominent frequency modulation, even though time resolution in our study was about 10 time more coarse than for Hankins \& Eilek (2007). The direct comparison between GPs from B1821-24A and the Crab pulsar would require observations at higher frequencies (where ISM scattering is negligible) and much better timing resolution.

Within one GP, the position angle of linear polarization and the sign/degree of circular polarization can vary greatly from patch to patch (see Fig. 4.10). On average, individual patches have a higher degree of linear polarization than band-averaged GPs (Fig. 4.9, middle). The same is true for circular polarization, the spread of fractional Stokes $\mathrm{V}$ is twice as big for patches than for bandaveraged GPs.

Patches arrive more or less uniformly throughout the observing band and the sum of all GPs in a given band has a rather smooth power-law $I(f)$. The power-law index for the sum of 835 GPs in L-band is $-1.8 \pm 0.2$. For 74 GPs in S-band it is $-2.2 \pm 0.2$ and for $75 \mathrm{GPs}$ at $820 \mathrm{MHz}$ it is $-2.0 \pm 0.7$. This agrees with previously measured spectral indices for the Crab pulsar (about -3 , with the scatter from -1.6 to -4.9 , Sallmen et al. 1999; Popov et al. 2006) and 
for B1937+21 (-3.1, Kinkhabwala \& Thorsett 2000). Interestingly, for both pulsars spectral index of GPs is comparable to the spectral index of the average emission in phase windows of GP arrival. In our case, GPs on the trailing edge of component P1 come from the region with much steeper spectra, with $\alpha$ of from -4 to -8 .

\subsection{Summary}

PSR B1821-24A has a complex multi-component average profile. The two brightest components, $\mathrm{P} 1$ and P2, are almost completely linearly polarized, but have very little fractional circular polarization. The phase-resolved position angle stays constant over the frequency range of $720-2400 \mathrm{MHz}$. Applying the Stokes tomography technique developed by Chung \& Melatos (2011), we find that the angles between the spin and magnetic axes and between the spin axes and observer's line of sight, $(\alpha, i)$ to be $\left(40^{\circ}-50^{\circ}, 10^{\circ}\right)$ or $\left(90^{\circ}, 60^{\circ}-90^{\circ}\right)$. The phase-resolved spectral index has approximately the same shape in the three observing bands, although phase-resolved spectra at higher frequencies tend to be slightly steeper. Trailing edges of P1 and P2 tend to have larger absolute values of spectral index. This effect is much more prominent for P1, where $\alpha$ can get as low as -8 . Interestingly, the region of the steepest spectra coincides with one of the phase windows of giant pulse arrival. This speaks in favor of the theory of GPs being created by induced scattering of radio waves by the plasma particles (Petrova 2006). Giant pulses also arrive on the trailing edge of component $\mathrm{P} 3$, but the $\mathrm{S} / \mathrm{N}$ of the average profile is too low there to measure the spectral index in our range of frequencies.

Giant pulses have intrinsic widths less than our time resolution of $10.24 \mu \mathrm{s}$. The energy of individual GP can be as high as 100 times the energy of the whole average profile. GP energies obey power-law statistics with a spectral index of -1.6. Owing to our large fractional bandwidth we were able to prove the hypothesis (Knight et al. 2006) that the spectra of GPs from B1821-24A consist 
of individual patches. Overall, GP spectra resemble those from the Main pulse Crab GPs, although our time resolution was not sufficient to make the direct comparison. Individual patches are substantially polarized with position angle and the sign of circular polarization changing randomly from patch to patch. The spectrum of the sum of all GPs selected in given band is a power-law with the index of about -2 . This is close to the spectral index of GPs from the Crab pulsar and B1937+21, $\alpha \approx-3$. However, for both B1937+21 and the Crab pulsar spectral index of GPs is comparable to the spectral index of the average emission in phase windows of GP arrival. In our case, GPs on the trailing edge of component P1 come from the region with much steeper spectra, with the index ranging from -4 to -8 . 


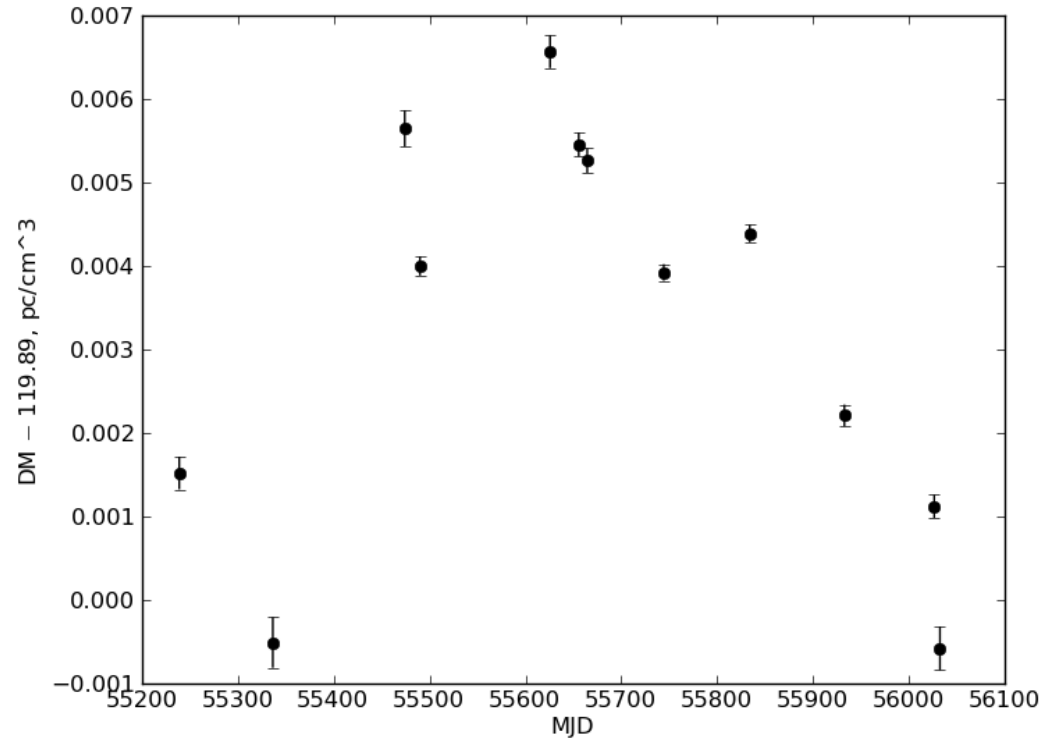

Fig. 4.1.- Dispersion measure variation over the course of the observations. Errorbars represent formal TEMPO errors. 

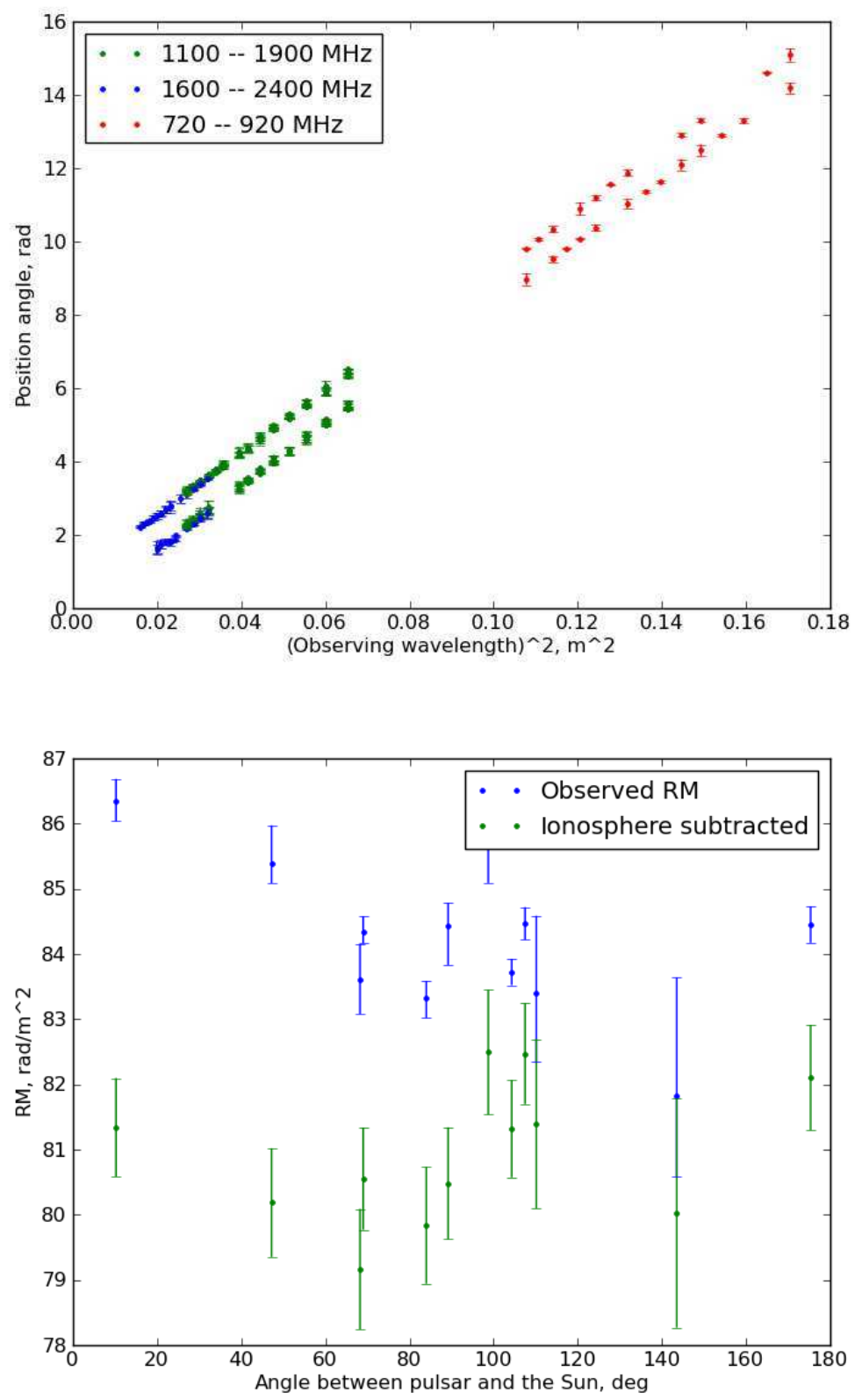

Fig. 4.2.- Top: Unwrapped position angle variation for the peak bins of the two brightest components in the average profile. The variation is linear, as expected for Faraday rotation, caused by magnetic field in ionized plasma in the interstellar medium and Earth's ionosphere. Bottom: Observed and ionosphere-subtracted rotation measure versus the angle between the pulsar and the Sun. 

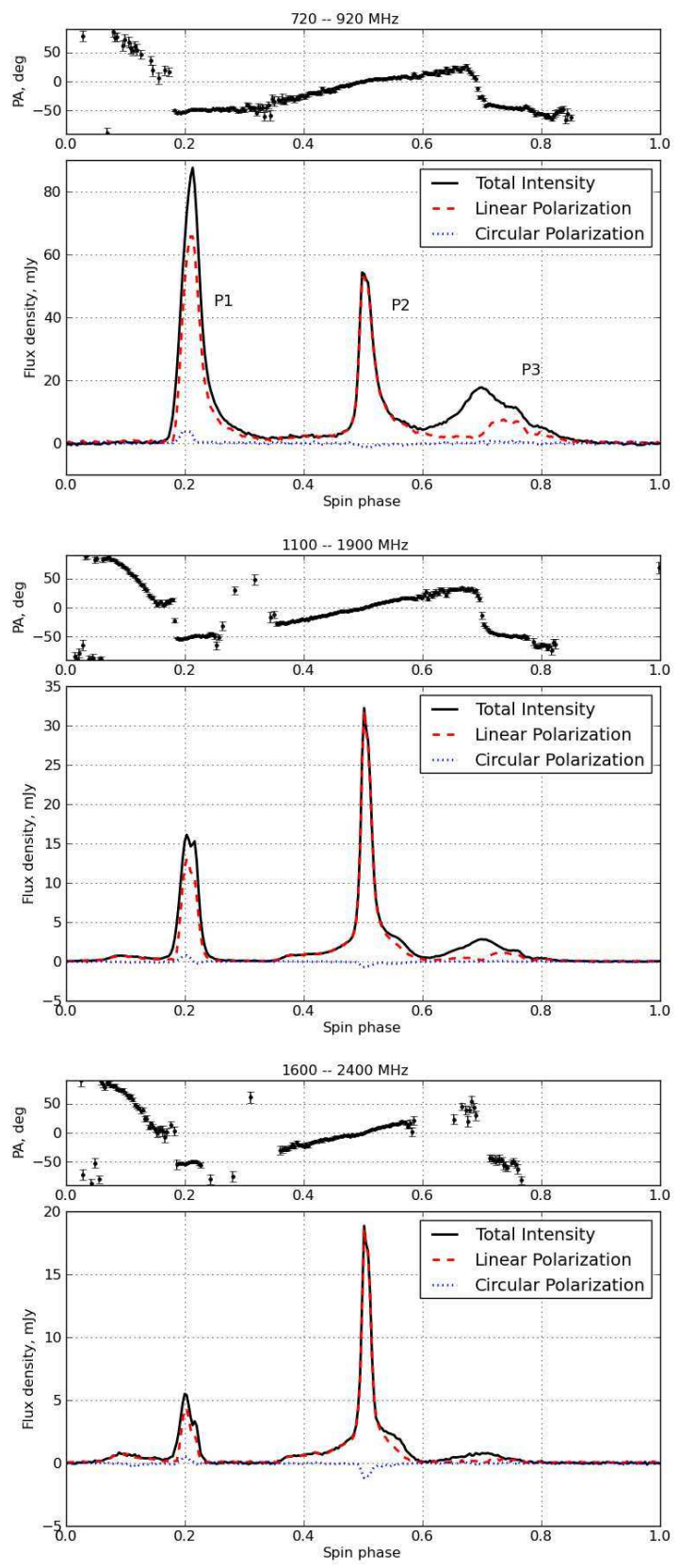

Fig. 4.3. - Average profile of B1821-24A together with position angle for $820 \mathrm{MHz}$ (top), L-band (1500 MHz; middle) and S-band (2000 MHz; bottom). 


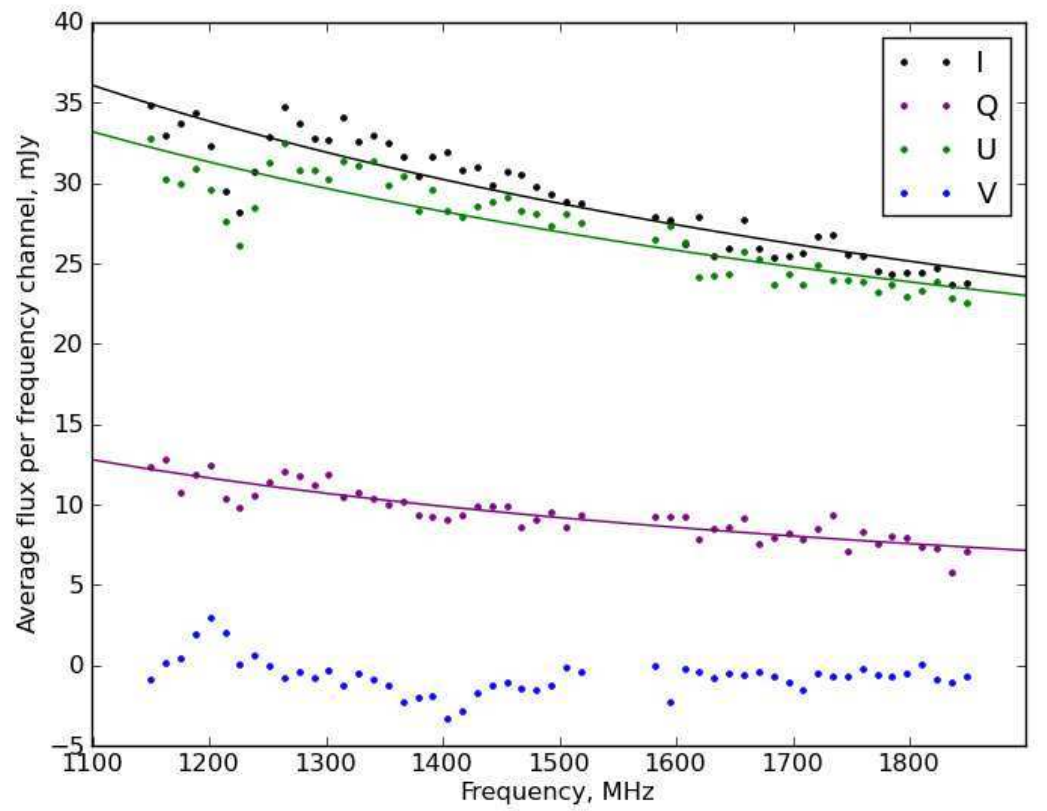

Fig. 4.4.- An example of a phase-resolved pulsar spectrum, for a bin with phase 0.5 for one observing day at L-band. Note the deviations from power-law around $1200 \mathrm{MHz}$. Such deviations were present in all observing sessions, had the same shape within each observing band and can be attributed to imperfect calibration. For all observing sessions the deviations from power-law for any Stokes component in any on-pulse phase bin were not bigger than $15 \%$ of total intensity at that phase and frequency. 


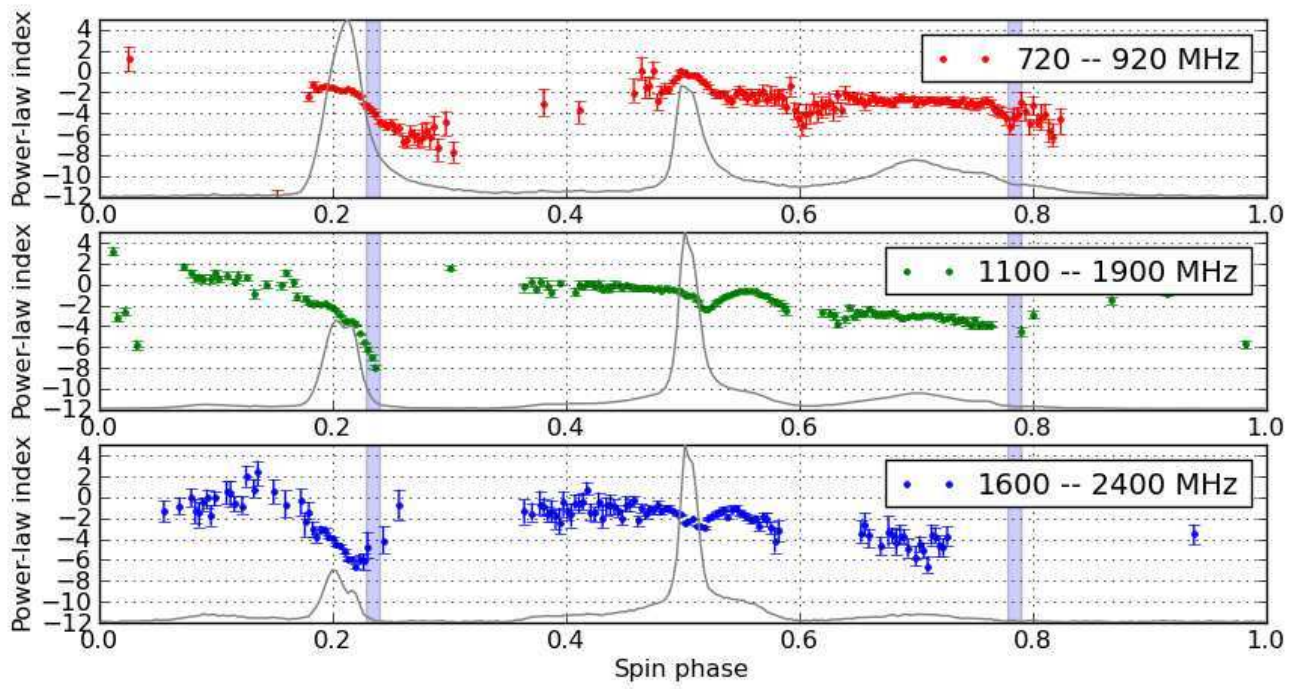

Fig. 4.5.- Phase-resolved spectral indices for three observing bands. The average profile for each band (grey line) is overplotted for reference. The shaded regions indicate the phase windows of giant pulse arrival. 


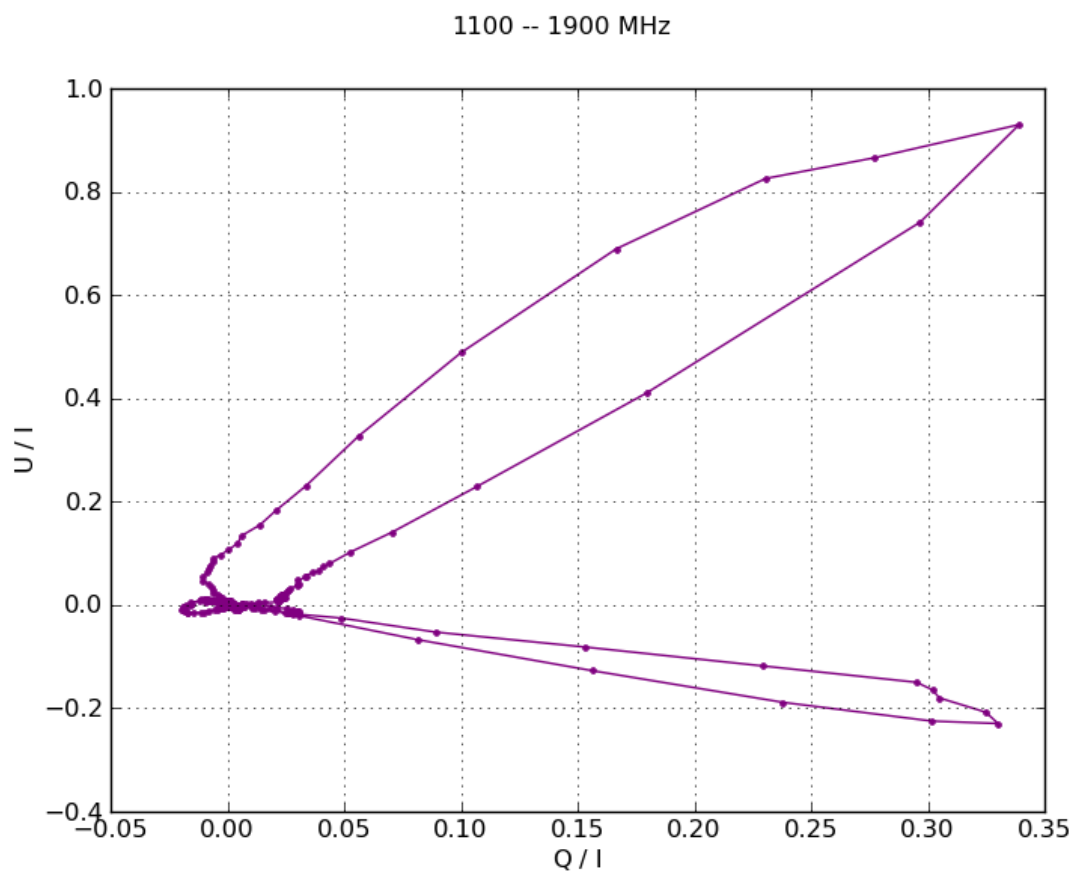

Fig. 4.6.- The phase portrait for Q-U Stokes components for L-band. Phase portraits of Stokes components can be a good diagnostic tool to infer a pulsar's magnetic geometry and orientation (see text for explanation). 


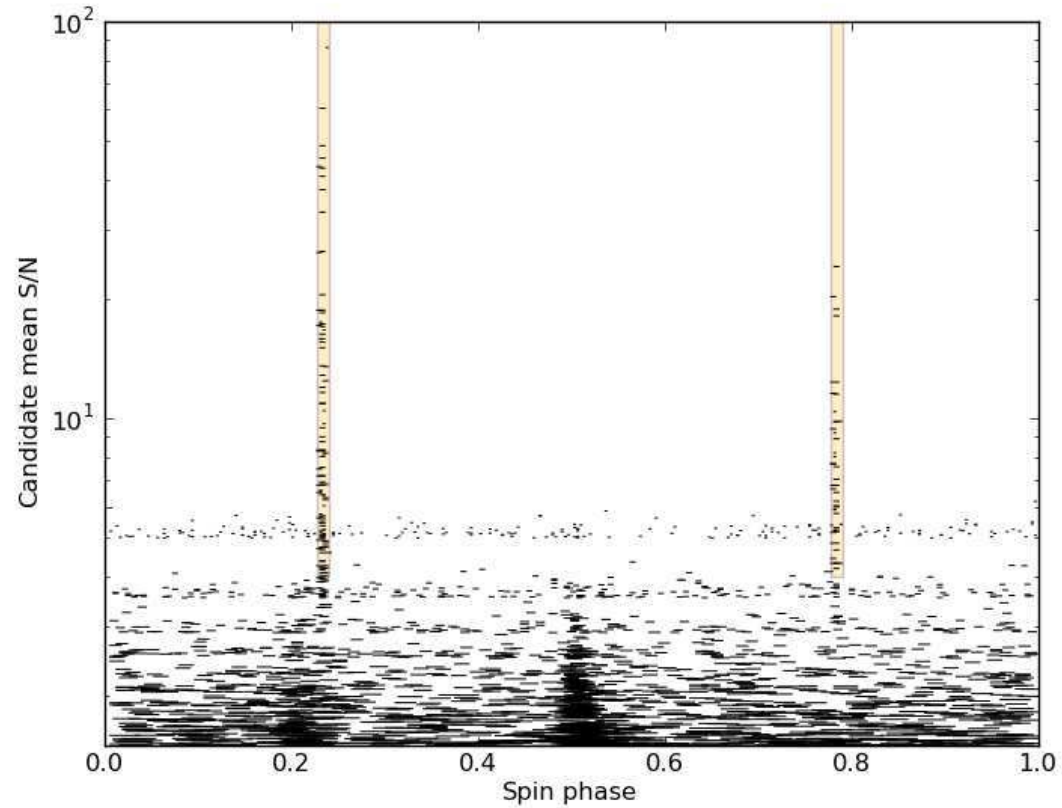

Fig. 4.7.- The single pulse candidates for one observing session at L-band. The candidates were selected by matched-filtering with a set of boxcars of different width. Each candidate is plotted as a bar in the phase window corresponding to its width. The signal to noise ratio of the candidates is averaged over the width of each. The clustering of events at a set of horizontal lines is due to a width-dependent selection threshold (see text for explanation). Candidates in shaded regions were selected as giant pulses. Broad faint candidates at phases 0.2 and 0.5 are the traces of the brightest normal pulses from components P1 and P2. 

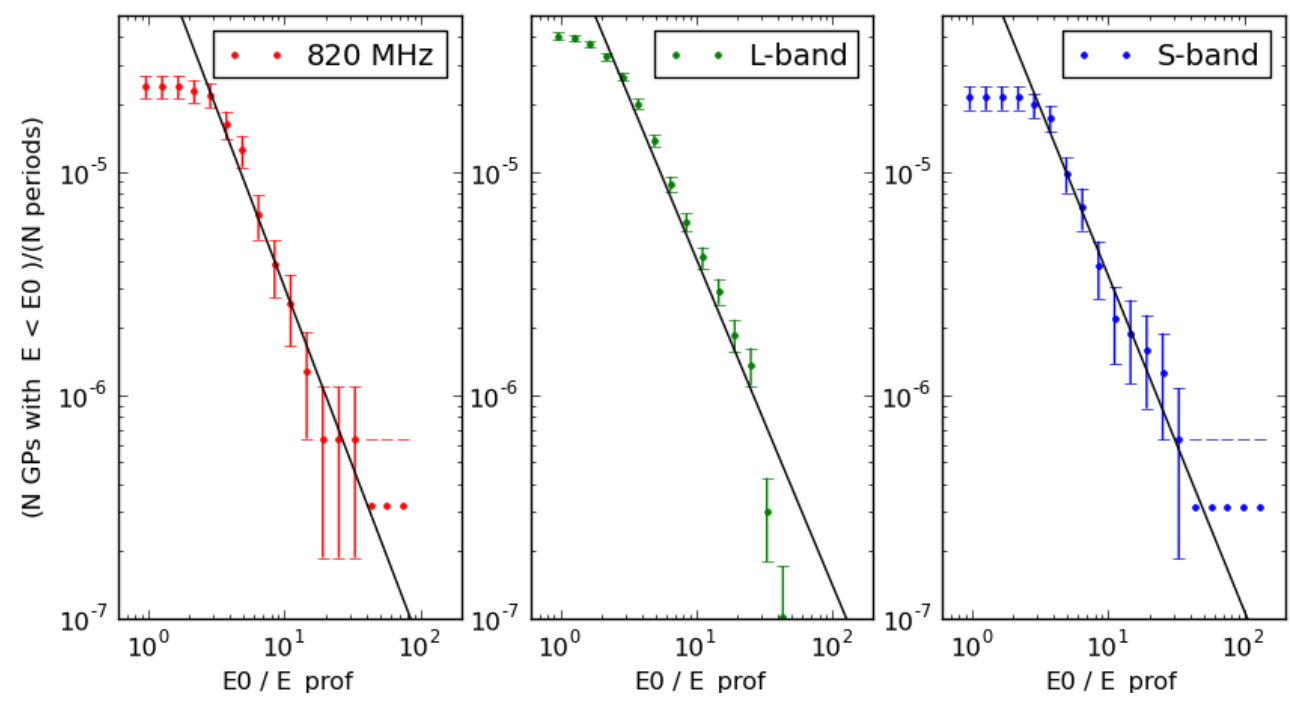

Fig. 4.8. - Cumulative energy distributions for GPs for $820 \mathrm{MHz}$ (left), L-band (middle) and S-band (right). Energies are measured relative to the energy of the average profile (integrated over a full pulse period). The flattening of the distributions at low energies is due to poor statistics close to the detection threshold and these energies were not included in the fit. The black lines denotes the best power-law fits. They have slopes of -1.6 for $820 \mathrm{MHz},-1.46$ for L-band and -1.6 for S-band. 

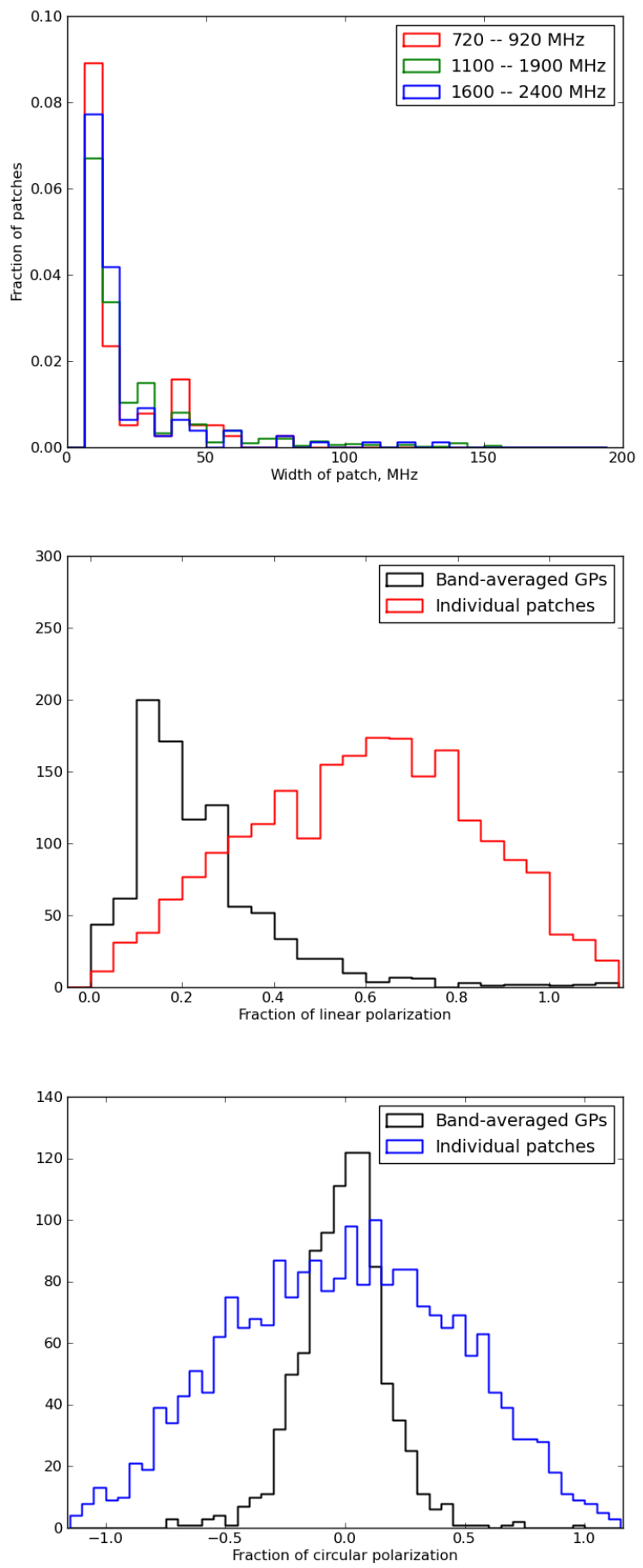

Fig. 4.9.- Left: The distribution of spectral widths of individual giant-pulse intensity patches for three observing bands. Middle: The distribution of fractional linear polarization for band-averaged GPs and individual patches. Individual patches tend to have a higher degree of linear polarization. Right: The distribution of fractional circular polarization for band-averaged GPs and individual patches. The spread of fractional Stokes- $V$ is twice as big for patches than for band-averaged GPs. 

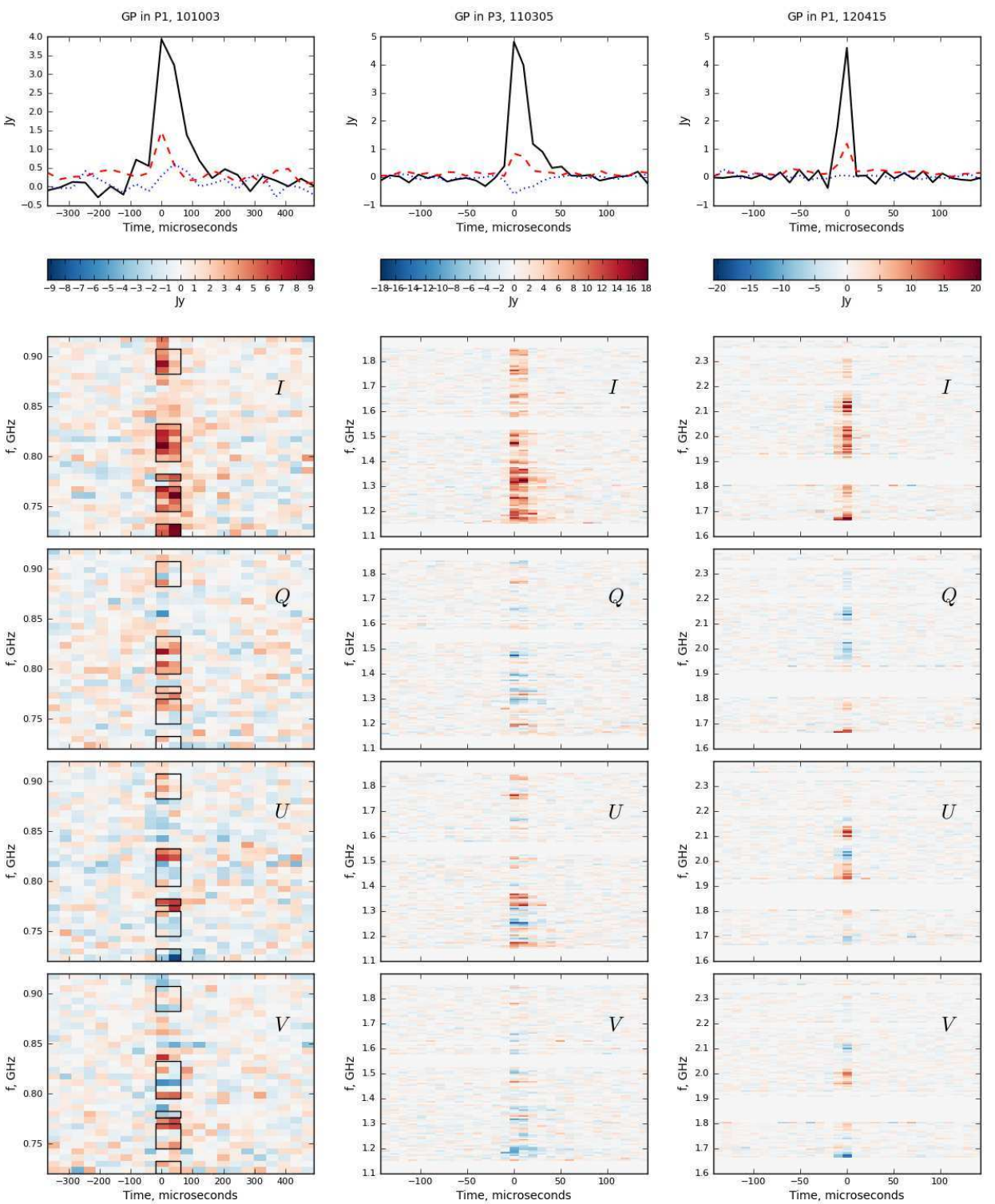

Fig. 4.10.- Examples of GPs from the three observed bands in four Stokes components. GPs are mostly unresolved in time and show clear exponential dispersive tails at lower frequencies. Spectral resolution in all cases is the same and comprises $6.25 \mathrm{MHz}$, an order of magnitude larger than the decorrelation bandwidth for interstellar scintillations. Thus, the frequency structure is pertinent to the GP emission mechanism. The size and position of frequency patches were obtained from widthaveraged $I(f)$ via a friends-of-friends algorithm. An example of patch localization is overplotted on the GP spectrum for $820 \mathrm{MHz}$ (left). Note that the position angle of linear polarization and the sign of circular polarization varies randomly from patch to patch. Thus, band-integrated GPs have a smaller degree of linear or circular polarization. 
Chapter 5

LOFAR Detection of Bright

Low-Frequency Narrow-Band

Pulses from Pulsars B0809+74

and $\mathrm{B} 1133+16$ 


\subsection{Introduction}

Rotation-powered radio pulsars display a variety of observational phenomena which must be explained by a comprehensive model describing the pulsar emission mechanism. Though their integrated, i.e. "cumulative", pulse profiles are often very stable, the intensity and shape of individual pulses vary significantly from pulse to pulse. The spectrum and characteristic timescale of this variability also covers a large range of parameter space. For example, some radio pulsars exhibit nulling, where the radio emission appears to temporarily cease (e.g., Herfindal \& Rankin 2009); some show quasi-periodic, intermittent on-off states (Kramer et al. 2006; Lyne et al. 2010) Others, like the so-called rotating radio transients (RRATs) are detectable only through their sporadic radio bursts (McLaughlin et al. 2006), though they likely do not represent a distinct class of neutron stars (Keane et al. 2011). All these phenomena occur on timescales which vary from the pulse period up to decades (e.g., Lyne et al. 2010). On timescales shorter than the pulse period, some pulsars exhibit microstructure (at $\lesssim 100 \mu$ s timescales, see, e.g., Lange et al. 1998; Popov et al. 2002), and giant pulses (GPs) with widths of a few $\mu$ s (e.g., Johnston \& Romani 2002; Knight 2006). Giant pulses from the Crab pulsar are known to be composed of a number of unresolved nanopulses with the duration of $<0.4$ ns (Hankins et al. 2003; Hankins \& Eilek 2007).

The aforementioned phenomena have mostly been studied at radio frequencies $\gtrsim 100 \mathrm{MHz}$, with most studies at around $\sim 1.4 \mathrm{GHz}$. This is mainly because the combination of pulsar spectra and effects related to interstellar propagation make the 1-2 GHz band an ideal range for pulsar observations. Pulse broadening due to intra-channel dispersion and scattering hampers detailed single-pulse studies at lower frequencies. Yet, strong individual pulses were reported in a series of papers by Kuzmin and Ershov (see, e.g., Kuzmin 2006, and references therein) from bright nearby pulsars, B0031-07, B1112+50, B0656+14, and J1752+2359 from observations at $112 \mathrm{MHz}$ with the Large Phased Array in Puschino (Russia). They quailified them as giant-pulse emission following the 
conventional definition of GPs as pulses with energies $>10\langle E\rangle$, where $\langle E\rangle$ is the energy of the average profile, though wider than traditional giant pulse. Weltevrede et al. (2006) performed a detailed analysis of single pulses of B0656+14 at higher frequencies of 327 and $1380 \mathrm{MHz}$ and found two pulse populations: bright "spiky" pulses and broader weaker ones, when the spiky emission can be as strong as giant pulses. Recent low-frequency study by Karuppusamy et al. (2011) of B0031-07, B1112+50, and B1133+16 at 110-180 MHz with the Westerbork Synthesis Radio Telescope showed that these pulsars exhibit large intensity variations that result in narrow and bright pulses.

Due to the strong frequency dependence of the Galactic synchrotron background $\left(\nu^{-2.6} ;\right.$ Lawson et al. 1987), its contribution to the system temperature at low frequencies $<100 \mathrm{MHz}$ is very significant. The ionosphere also becomes an important factor, producing an extra frequency-dependent pulse delay. Nonetheless, the very low-frequency range is interesting for pulsar studies because of pulse-profile evolution, which can become quite dramatic towards the lowest frequencies (Hassall et al. 2012), and because of potential for phenomena not seen at higher frequencies (or which become more pronounced at low frequencies). For example, studies performed with the Ukrainian "T"-shaped radio telescope, UTR-2, found occasional strong individual pulses from five pulsars - B0809+74, B0834+06, B0943+10, B0950+08, and B1133+16 - in the frequency range 18-30 MHz (Ulyanov et al. 2006). These pulses, termed "anomalously intensive pulses" (AIPs) occur quite rarely — in only a few percent of the pulse periods, are 10-15 ms wide (though finer structure may exist), and have peak flux densities $>20$ times greater than that of integrated pulse profiles. Their spectra were also reported to be narrow-band, with emission located in either one or a few narrow frequency channels of $1.5-5 \mathrm{MHz}$ wide (Ulyanov et al. 2006, 2007).

Here we report the first broadband low-frequency study of single pulses of the pulsars B0809+74 and B1133+16, using the Low Frequency Array (LOFAR) telescope to cover a large fractional bandwidth of $48-\mathrm{MHz}$ at frequency 
$<90 \mathrm{MHz}$. In Section 5.2 we describe our observing setup and processing pipeline and pulse selection approach. In Section 5.3 we provide the results on pulse energy and peak flux density distributions, pulse spectra and compare observed pulses with giant pulses. In Section 5.4 we speculate about possible origin of narrow-band pulses in the radio emission of the PSR B0809+74 at very low frequencies, and in Section 5.5 we give a summary of our work.

\subsection{Observations and Data Reduction}

We carried out a number of LOFAR commissioning observations of pulsars B0809+74 and B1133+16 at the end of 2011 using the Low Band Antennas (LBAs) in the LBA_OUTER array mode, where the 48 outermost dipoles in each LBA field are used. The instantaneous bandwidth of $46.875 \mathrm{MHz}$ centered at the frequency $<50 \mathrm{MHz}$, depending on the observing setup for particular observation, was split into 240 subbands of 32 channels each and originally sampled with the time interval of $491.52 \mu$ s (see Table 5.1). Each observation was the coherent sum of the 6 "Superterp" stations, CS002-CS007. A detailed description of LOFAR's pulsar observing modes and the online data reduction pipeline is given in Stappers et al. (2011).

The data were converted from the LOFAR beam-formed format to the PSRFITS data format and then processed with the DSPSR software suite ${ }^{1}$ (van Straten \& Bailes 2011) to form both an integrated profile as well as single-pulse integrations. Although several observations were taken and processed, most of them were strongly contaminated by radio frequency interference (RFI), especially at the very low frequencies $<30 \mathrm{MHz}$. Thus, for the subsequent analysis we chose the best observation of PSRs B0809+74 and B1133+16 of 57-min each which were mostly RFI-free. Pazi from the PSRCHIVE package ${ }^{2}$ (Hotan et al. 2004) was used to excise any remaining RFI.

\footnotetext{
${ }^{1}$ http://dspsr.sourceforge.net

${ }^{2}$ http://psrchive.sourceforge.net
} 


\subsubsection{DM tweaking}

At LOFAR's lowest frequencies the dispersion measures (DMs) from the available pulsar ephemerides are not accurate enough to properly dedisperse the data. For an initial single-pulse search, we scrunched (i.e. integrated) all frequency channels and calculated the signal-to-noise ratio of the peak, keeping only strong pulses. We selected 82 pulses stronger than $15 \sigma$ for the pulsar B0809+74 and 170 pulses $>13 \sigma$ for the pulsar B1133+16. Consequently, we used pdmp from the PSRCHIVE package to determine the best DM for each of the pulses. Applying a least-squares fit to the strongest pulses, we then determined the best average DM for each pulsar, namely $5.752 \mathrm{pc} \mathrm{cm}^{-3}$ for B0809+74 and $4.844 \mathrm{pc} \mathrm{cm}^{-3}$ for B1133+16, with the DM jitter between the pulses being on the order of $0.002 \mathrm{pc} \mathrm{cm}^{-3}$ for both pulsars. These new DM values differed by 0.001-0.002 pc $\mathrm{cm}^{-3}$ from old values and were used to re-run dspsr to dump single pulses for the subsequent analysis.

Table 5.1: LOFAR observing setup

\begin{tabular}{lcc}
\hline Pulsar & B0809+74 & B1133+16 \\
\hline ObsID & L32655 & L36847 \\
Date & Oct 26, 2011 & Dec 7, 2011 \\
Array & LBA Outer \\
Number of stations & 6 \\
Stations used & CS002-007 (Superterp) \\
Frequency filter range (MHz) & $10-90$ \\
Center frequency (MHz) & 37.9852 \\
Bandwidth (MHz) & 46.87568 \\
Observing time (min) & 57 \\
Number of subbands & 240 \\
Subband width $(\mathrm{kHz})$ & 195.3125 \\
Number of channels/subband & 32 \\
Channel bandwidth (kHz) & 6.1035 \\
Sampling time $(\mu \mathrm{s})$ & 491.52 \\
Polarization & Stokes $I$ \\
\hline
\end{tabular}



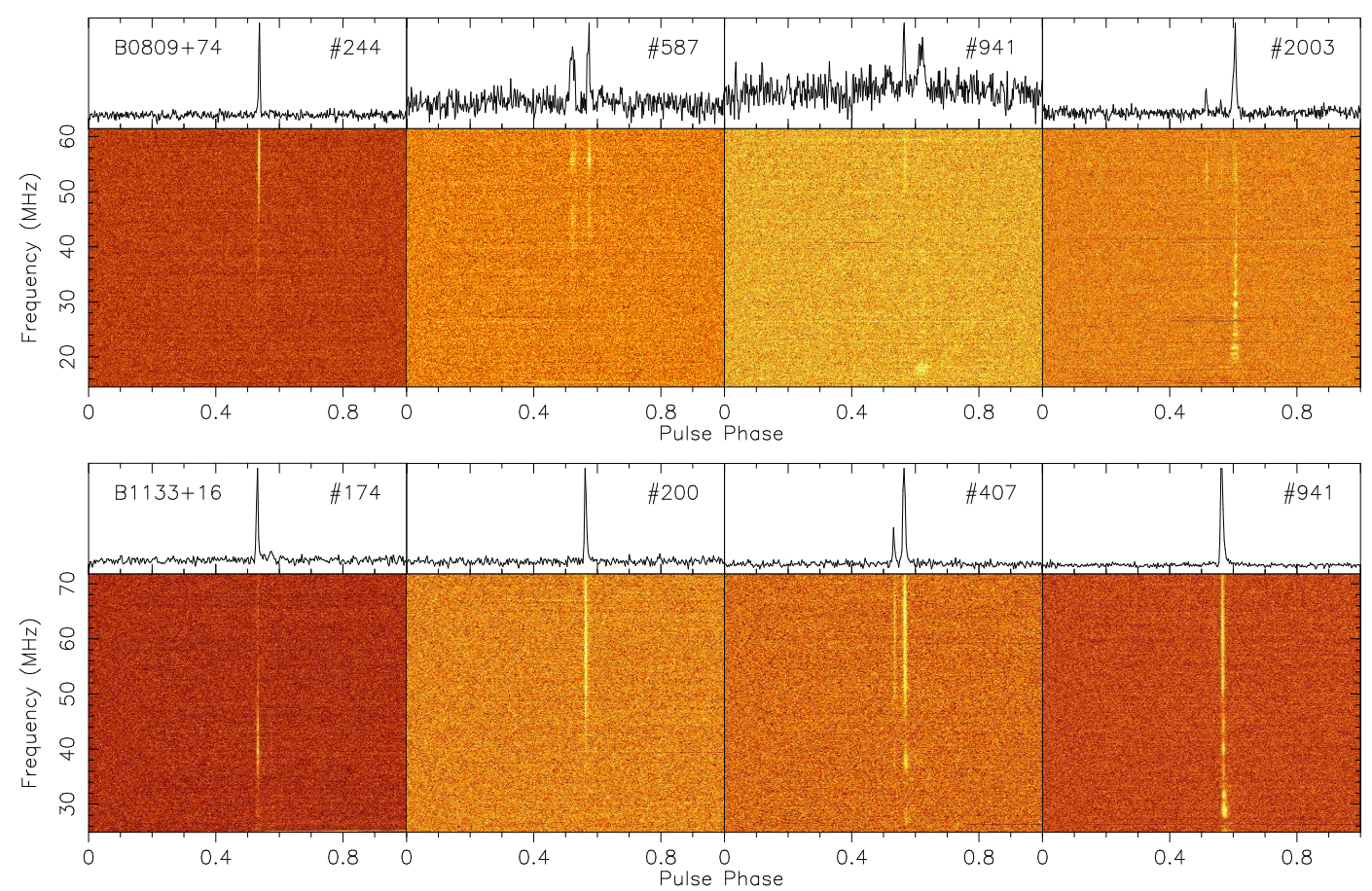

Fig. 5.1. - Examples of characteristic spectra and profiles of individual pulses of the pulsars B0809+74 (top row) and B1133+16 (bottom row). The number in the topright of each panel shows the pulse number from the beginning of the observation. Each spectrum is comprised of 480 channels of $97.656 \mathrm{kHz}$ each. There are 438 bins per period for the plots of B0809+74, and 302 phase bins for B1133+16.

\subsubsection{Single-pulse selection}

Figure 5.1 shows the typical spectra of individual pulses from pulsars B0809+74 and B1133+16. As was originally reported by Ulyanov et al. (2006), many of the pulses can be seen to exhibit narrow-band structure. Pulsar emission is typically broadband on the band scales up to few hundreds of $\mathrm{MHz}$ (the capacities of current receivers/backends) This statement is also true in the case of the pulsars B0809+74 and B1133+16, as observed at high frequencies.

To detect weaker narrow-band pulses which would be otherwise missed when searched for over the integrated bandwidth, we developed a 2-D single-pulse search tool operating in time-frequency space, similar to the standard singlepulse searches used in pulsar surveys (e.g., McLaughlin \& Cordes 2003). We 
convolved each period with a 2-D top-hat function with different discrete widths in frequency and time and calculated the signal-to-noise ratio $(\mathrm{S} / \mathrm{N})$ of the time/frequency averaged signal, focusing on the phase range where emission is present in the cumulative pulse profile. However, this search technique was not consistent enough in detecting the proper width of emission patches in the spectra, as the search criteria was based on the average flux density in the emission region and sometimes brighter narrower structure in the patch got selected. Also, the performance of this method was quite slow especially for top-hat functions of large widths.

Instead, we implemented another search technique that provided better, more consistent results, allowing us to determine the size and location of the features in the spectra more accurately. Closer inspection of the large sample of both pulse spectra and their integrated profiles showed that even when there was only one bright, narrow-band emission patch in the pulse spectrum, its contribution to the integrated profile in the entire band was noticeable. Therefore, we divided our search into two parts. First, to find the location of the single pulse components in pulse phase, we performed the standard single-pulse search technique in the time-domain on each individual pulse (up to 3 drifting subpulse components for the pulsar B0809+74, and two components for the pulsar B1133+16). To speed-up the search and minimize the noise fluctuations in the profile we scrunched the profile in phase by a factor of 6 for B0809+74 and a factor of 8 for B1133+16, providing an initial time resolution of about 2.95 and $3.93 \mathrm{~ms}$ for pulsars B0809+74 and B1133+16, respectively. We formed the mean spectrum of each pulse profile component by averaging the signal within the component's width. Then, each mean spectrum was searched for features above a $5 \sigma$-threshold. The size of the emission patches in the frequency domain was determined by finding peaks (above the $5 \sigma$-threshold) in the mean spectrum and then walking up and down in frequency until the signal dropped below the threshold. The corresponding frequencies determine the edges of an emission patch. The search was repeated until all emission above the threshold 
was accounted for. To minimize the influence of the noise fluctuations of our spectrum search, we scrunched our original spectrum by 128 channels, providing a spectrum resolution of $781.25 \mathrm{kHz}$. A similar search was also performed on the OFF-pulse phase window of the same size as for the ON-pulse one. The purpose of the OFF-pulse phase window search was to have "one-to-one" comparison between $\mathrm{ON}$ and $\mathrm{OFF}$ distributions obtained the same way. If there is no pulsar signal in our ON-pulse window, then search will find the "highest" noise patch there, so, we should compare it with the OFF-pulse distribution obtained the same way on the OFF-pulse window. Also, if allowed us to check for any outstanding values is there was some terrestrial interference left.

\subsubsection{Bandpass correction}

Detection of emission patches and their frequency widths can be potentially affected by different gain in the overall LBA band. The generic LBA sensitivity curve is not flat and has a somewhat gaussian shape peaking at about $58 \mathrm{MHz}$ (van Haarlem et al. 2012, in preparation). The true instantaneous sensitivity can also vary somewhat depending on the Galactic background, zenith angle, time of the day, RFI, and even weather. Currently, a good model for LBA sensitivity is not available. Still, to correct for the bandpass gain we calculated the average spectrum over the whole course of our observations using the raw data. Though the pulsar signal was present in the data, its signal was dispersed significantly at such a low observing frequency (about 11 and $2 \mathrm{~s}$ for B0809+74 and B1133+16, respectively, at the lowest observing frequency channel). We calculated the correction coefficients for different channels as the inverse of the ratio between spectrum value of the channel to spectrum's maximum, and applied them during the conversion to PSRFITS format. Such correction was only performed for the $\mathrm{B} 0809+74$ data as the raw data for $\mathrm{B} 1133+16$ were already deleted. However, we compared the results of our searches for the pulsar B0809+74 using the bandpass-corrected data and the data without correction, and they turned out to be very similar, meaning that different sensitivity over 
the LBA band is not the crucial factor contributing to the pulse search results.

\subsection{Results}

Figure 5.1 shows some typical pulses from B0809+74 (top row) and B1133+16 (bottom row). Each panel contains an individual pulse profile (top panel) and its pulse spectrum (bottom panel). For both pulsars single pulse emission can occur in either leading or trailing components, or both components at the same time, or in case of the pulsar B0809+74 sometimes in three components (see, e.g., pulse \#941 on Figure 5.1) from three drifting subpulse bands. The frequency structure of the pulse spectra can also be quite different both from pulse to pulse and even between components within the same pulse, especially for the pulsar B0809+74. For example, pulse \#941 shows a very narrow emission patch at $19 \mathrm{MHz}$ in the second component, but the strongest emission from the other two components occurs at the higher frequencies. Other examples (e.g. pulse \#2003) show very broadband structure comprised of a number of smaller individual patches with the maximum tending to be at lower frequencies $(<30 \mathrm{MHz})$. Most often the pulse spectra of both components are similar as in the case of pulse \#587 and consists of $1-3$ patches of $\sim 5-15 \mathrm{MHz}$ each with the maximum above 35-40 MHz. On average, the pulse spectra of B0809+74 are quite narrow and occupy only half of the band. We note, though, that we can not truly measure the frequency width of the emission patch extending beyond the high-frequency end of our observing bandwidth. The pulse spectra of $\mathrm{B} 1133+16$ are much broader in comparison with that of B0809+74 and in most cases emission occurs at higher frequencies. Low-frequency emission and pulses with several patches in the spectra do occur from B1133+16 (see, e.g., pulses \#407 and \#941), but not as often as they are seen from B0809+74. 


\subsubsection{Pulse energy distributions}

Pulse energy distributions for B0809+74 and B1133+16 are shown in Figure 5.2. The energy is calculated for the emission patches of every pulse component in the profile rather than over the entire band due to the presence of narrow-band pulses. We normalize the patch energy by the energy of the average profile in the entire band allowing a direct comparison of energy values obtained by other studies at different frequencies. Distributions are shifted with respect to 0, skewing towards higher energies as we search for the highest peak flux density on the spectrum averaged over the patch phase window. For better comparison between $\mathrm{ON}$ - and OFF-pulse distributions, we used exactly the same search technique with the threshold of $1 \sigma$ for both ON and OFF-pulse phase windows (yellow and green histograms in Figure 5.2). It is clear that distributions are very similar at low energies with a slightly smaller number of low-energy patches in the ON-pulse window. A few positive outliers in the OFFpulse histogram indicate the presence of RFI but their number is insignificant. We also show the ON-pulse histogram for the strong patches with the spectral peak flux density $>5 \sigma$. The roll-off of the histogram at low energies represents the selection bias for patches with peak fluxes close to the detection threshold. The apparent larger fraction of stronger patches than $1 \sigma$-patches for the same energy, is artificial and represents the fact that the overall number of detected patches is much larger for the $1 \sigma$ threshold than for the threshold of $5 \sigma$.

We do not see any apparent break in the pulse energy distributions for either pulsar which might indicate the presence of two different populations of emitted pulses. However, for B1133+16 there might be a break at energies around $1\langle E\rangle$ which is seen more clearly in the ON-pulse distribution for the $1 \sigma$ threshold. For B0809+74 the absence of a visible break does not necessarily exclude one as it could be hidden in the noise and/or the distribution slopes for weak and strong pulses could be close to each other.

Typically the pulse energy for normal pulsar pulses follows lognormal or normal (i.e. gaussian) statistics (see, e.g. Cairns 2004), though other more complex 
multi-peaked distributions have been observed (Burke-Spolaor et al. 2012). Giant pulse emission follows a power-law distribution of energies (see, e.g. Popov \& Stappers 2007; Soglasnov et al. 2004). To determine the type of pulse energy distributions for $\mathrm{B} 0809+74$ and $\mathrm{B} 1133+16$ at low frequencies we fit normal, log-normal and power-law distribution with a low-energy cutoff. The best-fit parameters for each of the distributions are given in Table 5.2. For our fits we excluded the very high-energy tail of the distributions where the statistics are poor. Also, as the roll-off at low energies is due to our detection technique and is not intrinsic to the pulsars, for log-normal and power-law fits we used only the bins with energies larger than the energy which corresponds to the peak of the distributions. To optimize the fit parameters we used the Levenburg-Marquardt algorithm and minimized the $\chi^{2}$ value. To estimate the goodness of fit we used both the chi-squared and the Kolmogorov-Smirnov (KS) test. For both pulsars the Gaussian distribution provided a poor fit to the observations, and the corresponding significance probability for both the $\chi^{2}$ and KS-test is low ${ }^{3}$ The lognormal distribution provides the best goodness of fit especially in the case of B0809+74. However, fitting a power-law distribution to the high-energy tail for energies $>2.4\langle E\rangle$ provides better results. In general, the significance of the fit is not high enough for either pulsar to reject the lognormal or the power-law distribution. Better statistics are needed.

\subsubsection{Peak flux densities}

In Figure 5.3 we present the distributions of the peak flux densities of the emission patches for all components for B0809+74 and B1133+16. At peak flux densities of about $12\langle S\rangle$ and $3\langle S\rangle$ for B0809+74 and B1133+16, respectively, where $\langle S\rangle$ is the peak flux density of the average profile, there is an implicit break in the distributions that might indicate the presence of two pulse populations. Obviously, there are a number of very bright pulses that might represent

\footnotetext{
${ }^{3}$ Though for B1133+16 the $P_{\mathrm{KS}}$ is quite low (0.035), the KS-statistic (or so-called D-value) is very large as well $(\sim 0.7)$, so one can easily reject the hypothesis of the Gaussian distribution.
} 
a separate group of exceptionally bright pulses or be the high flux density part of the general pulse distribution. These observations agree with Ulyanov et al. (2006) who reported anomalously intensive pulses, or AIPs, stronger than $20\langle S\rangle$ from pulsars B0809+74, B0834+06, B0950+08, B0943+10, and B1133+16 at the frequency of $18-30 \mathrm{MHz}$ using the UTR-2 radio telescope. Later on, another sixth pulsar B0823+26 was added to this sample (Ulyanov et al. 2007). They report that these strong pulses occur only $5-10 \%$ of the time. In our observations we obtained a similar occurrence rate for B0809+74 of about 14\%, but a somewhat lower rate of $0.6 \%$ for $\mathrm{B} 1133+16$. For $\mathrm{B} 1133+16$ this can be explained by the larger frequency width of the emission patches and the larger overall band, thus increasing the peak flux density of the average profile. The strongest registered pulses in our observations had peak flux densities of $108\langle S\rangle$ and $27.5\langle S\rangle$ for B0809+74 and B1133+16, respectively.

To investigate the properties of the bright pulses in more detail we calculated the average profiles comprised of only pulses larger than some threshold for both pulsars. Choosing different thresholds we stacked the corresponding profiles on top of each other including more weaker pulses. The corresponding plots for B0809+74 and B1133+16 are shown in Figure 5.4. The profile on the top shows the average profile including all pulses. The profile at the very bottom is comprised of only strongest pulses $>40 \sigma$. The larger noise fluctuations for profiles near the bottom is due to the small number of such pulses summed. It is clearly seen that strong pulses from B0809+74 are narrower and tend to come in the inner part of the average profile. The number of pulses that occur at the phase of the second component is somewhat larger, but not as extreme as in the case of B1133+16, where more than $50 \%$ of the pulses stronger than $20 \sigma$ come in the second component. This result is different from what i42s seen at frequencies from $110-180 \mathrm{MHz}$, where the amplitude of the first component is somewhat larger than the second (Karuppusamy et al. 2011). As one can see for B0809+74, when adding less strong pulses into the profile, a small weaker shoulder starts forming at the trailing edge of the second component, 
and slightly less pronounced at the leading edge of the first component. This could further support the presence of a weaker pulse population in the emission from $\mathrm{B} 0809+74$, but most likely it is due to a contribution from adjacent drifting subbands.

\subsubsection{Pulse spectra}

As mentioned in Section 5.3 some of the pulses from B0809+74 show very narrow-band frequency structure, particularly at the very low frequencies, with some of the emission patches being as narrow as $1 \mathrm{MHz}$. The emission from $\mathrm{B} 1133+16$ on the contrary, is much broader though truly narrow patches below $30 \mathrm{MHz}$ do occur. Ulyanov et al. (2006) reported the narrow frequency structure for all of the pulsars in their sample including B0809+74 and B1133+16. This can be partly explained by the fact that their observing band lay below $30 \mathrm{MHz}$ and thus the bulk of the emission at higher frequencies was missed and they saw only the small fraction of the emission below $30 \mathrm{MHz}$. To characterize the parameters of the emission patches for B0809+74 and to check if there is any preferential frequency/width of their emission, we made histograms of their central emission frequencies and frequency widths. It is evident from the left panel of Figure 5.5 that most of the patches occur at higher frequencies, a fact that is probably due to the pulsar's intrinsic spectrum, as we bandpass-corrected our data. There is not a gap in emission frequency or a significant decrease in emission at a particular frequency that would indicate the presence of unique emission at lower frequencies. In the right panel one can see the histogram of the frequency widths of emission patches. There is a clear indication that there are fewer broad emission patches for B0809+74. The significant number of very narrow patches may not reflect the true picture as some of those weaker patches could instead be part of another patch but detected as a separate one by the search pipeline. We, hence, made a histogram for stronger patches with spectral peak flux density $>10 \sigma$. For stronger patches there is a clear indication that they tend to have widths of about $3 \mathrm{MHz}$, but patches as wide as $16 \mathrm{MHz}$ and 
as narrow as $1 \mathrm{MHz}$ do also occur.

\subsubsection{Giant pulses?}

Both pulsars, but especially B0809+74, show remarkably bright individual

pulses as can be seen in Figure 5.3. Ulyanov et al. (2006) called them "anomalously intensive pulses" and we agree that their peak flux densities are very high. However, there are other pulsars known to emit very strong individual pulses, such as GPs from the Crab pulsar B0531+21 (Staelin \& Reifenstein III 1968), the original millisecond pulsar B1937+21 (see, e.g. Kinkhabwala \& Thorsett 2000), and a few other millisecond pulsars (see, e.g. Knight 2006) or "spiky emission" from B0656+15 (Weltevrede et al. 2006), which is more than 100 times brighter than the average profile at $327 \mathrm{MHz}$. Kuzmin (2007) also reported individual pulses with peak flux densities hundreds of times larger than the peak flux density of the average profile from the pulsars B0031-07, B1112+50, B0656+14, and J1752+2359. Strong pulses B1133+16 were also reported by Karuppusamy et al. (2011) at frequencies from 110-180 MHz. Hence, do the anomalously intensive pulses truly represent a new class of pulsar emission or it is just a manifestation of already known phenomenon but at very low radio frequencies?

As one can see from Figure 5.2, the maximum energies of emission patches from both pulsars are less than $10\langle E\rangle$, which is used as a conventional working definition of the threshold for giant pulses (Cognard et al. 1996). Using this definition, the pulses we observed do not qualify as GPs. Giant pulses are also known to follow a power-law distribution in pulse energy (see, e.g. Popov \& Stappers 2007; Soglasnov et al. 2004). In their recent work Ul'yanov \& Zakharenko (2012) presented the pulse energy distributions for B0809+74, B0943+10, B0950+08, and B1133+16 and claimed that they are power-law and similar to those observed from millisecond pulsars with GPs. However, they did not show their best fits to the observed distributions, nor the statistical errors of their histogram bins. Moreover, their distributions were derived for the very 
narrow frequency band from 23-24 MHz which might not truly represent the pulse emission properties. Therefore, the question about pulse energy distribution of pulses with AIPs remains open. In our present work we also could not differentiate between lognormal and power-law distributions for the B0809+74 and $\mathrm{B} 1133+16$. The significance of the fits is not large enough to reject one distribution in favor of the other.

In addition to very large peak flux densities, some of the pulses from B0809+75 show very narrow-band emission patterns at frequencies below 30-35 MHz. The frequency of $30 \mathrm{MHz}$ was in fact the high-frequency end of the observing bandwidth in the paper by Ulyanov et al. (2006) where they first reported AIPs. To consider the possibility that AIPs are indeed a unique class of narrow-band emission at low frequencies, we separated our analysis between all emission patches below $30 \mathrm{MHz}$ and those above $30 \mathrm{MHz}$. We only counted the patches that were completely below or above the threshold frequency of $30 \mathrm{MHz}$. The pulse energy and peak flux density distributions for patches below and above $30 \mathrm{MHz}$ are shown in Figure 5.6. The energies and peak flux densities of the patches are normalized by the corresponding energy or peak flux density of the average profile either below or above $30 \mathrm{MHz}$. The pulse energy distributions in the left panel clearly show that for a dozen patches below $30 \mathrm{MHz}$ their energies are very high compared with those at higher frequencies. These pulses are more a reflection of the facts that at the very low frequencies the emission in general is very weak, and these bright energetic pulses occur very rarely, thus the energy of the average profile is very small. For the distribution of peak flux densities we see that the bright pulses occur evenly at low and high frequencies. Due to small number statistics we can't really conclude if these energetic pulses represent a separate group or only the high-energy tail of the same distribution that describes the low-energy pulses as well. 


\subsection{The narrow-band emission of the PSR B0809+74}

We can not relate yet bright individual pulses from the pulsars B0809+74 and B1133+16 at frequencies below 65-70 MHz either to giant pulse phenomenon nor to pulsars that show "spiky" emission. Their energies and tendency to come closer to the inner edge of the profile component is similar to the spiky emission observed from the pulsar B0656+14 (Weltevrede et al. 2006) and B1133+16 at the frequency of 110-180 MHz (Karuppusamy et al. 2011). Nevertheless, the narrow-band spectra of some of the pulses from B0809+74 is quite unique and has never been observed before at higher frequencies. In this Section we speculate on the possible origin of such narrow-band pulses.

\subsubsection{ISM scintillations?}

The dynamic spectra for B0809+74 and B1133+16 are shown in Figure 5.7. These do not show characteristic scintles such as would be expected from diffractive scintillation. Using the empirical relationship between the decorrelation bandwidth $\Delta \nu_{\mathrm{d}}$, pulsar dispersion measure and observing frequency (Bhat et al. 2004), we find that $\Delta \nu_{\mathrm{d}}$ is likely $\lesssim 2 \mathrm{kHz}$ at the high frequency edge of our band for both pulsars and two orders of magnitude lower at the lower edge. Thus, all ISM scintillations will be completely averaged out in our data, as we used subbands $780 \mathrm{kHz}$ wide for our analysis. These estimates are in good agreement with values reported by (Smirnova \& Shishov 2008) from their observations with the BSA and DKR-1000 radio telescopes in Puschino at the frequencies of 41, 62, 89, and $112 \mathrm{MHz}$ for B0809+74. They found $\Delta \nu_{\mathrm{d}}$ to be $2 \pm 0.6,7 \pm 3$, $20 \pm 10,45 \pm 5 \mathrm{kHz}$ at the frequencies of $41,62,89$, and $112 \mathrm{MHz}$, respectively. For B1133+16, Malofeev et al. (1995) reported the decorrelation bandwidth to be $4.6 \pm 0.1 \mathrm{kHz}$ at a frequency of $102.7 \mathrm{MHz}$. For the thin screen model with a Kolmogorov spectrum of density inhomogeneties, at the frequency of $60 \mathrm{MHz}$, $\Delta \nu_{\mathrm{d}}$ would be $\sim 0.4 \mathrm{kHz}$, significantly smaller than the spectral width of our channels. 
We also estimated the characteristic time scale of refractive scintillations, $\tau_{\text {RISS }}$. Using the measurements of $\tau_{\text {DISS }}=450 \pm 70 \mathrm{~s}$ by Smirnova \& Shishov (2008) for B0809+74 at $89 \mathrm{MHz}$ and $\tau_{\text {DISS }} \sim 5$ s by Malofeev et al. (1995) for B1133+16 at $102.7 \mathrm{MHz}$, for a Kolmogorov spectrum we found that at our observing frequencies, $\tau_{\text {RISS }}=\tau_{\text {DISS }}\left(\nu / \Delta \nu_{\mathrm{d}}\right)=10-30$ days for B0809+74, and 4-6 days for B1133+16. Comparing the fluxes of average profiles from several observations separated by days and weeks, we indeed see flux variations caused by RISS. However, within 1-h observations used for the analysis in this paper, RISS are insignificant. Thus, appears highly unlikely that the narrow-band emission patterns are caused by any type of ISM scintillations.

\subsubsection{Ionosphere?}

In our LBA observations the coherent beam formed by six Superterp stations is about $1.2 \mathrm{deg}$ wide, and line-of-sights from compactly located stations within $300 \mathrm{~m}$ trace the same electron content in the ionosphere. The rate of change in the state of the ionosphere is long (mins-hours) compared to time scales of about a second when narrow-band pulses follow each other. Therefore, the ionosphere is also very unlikely to be the cause of narrow-band structure from B0809+74.

\subsubsection{Strong plasma turbulence models}

In Figure 5.8 we show the dependence of the emission patch frequency width on their central frequency for the strong patches from B0809+74 with energy $E>2\langle E\rangle$. Though data points are somewhat scattered, they qualitatively lie on the line, $\Delta f / f_{c} \sim 0.15$. This agrees with the prediction from the strong plasma turbulence (SPT) model of radio pulse emission (Weatherall 1998) which predicts narrow-band radiation with $\Delta f / f \sim 0.1-0.2$. At our observing frequencies from $20-70 \mathrm{MHz}$ we would expect pulses with bandwidths of $\sim 2-14 \mathrm{MHz}$, exactly what we observed for B0809+74 (see Figure 5.5). Pulse frequency 
widths should also increase with frequency, and this again what we observe, with broader emission patches occurring at higher frequencies.

The SPT model also describes well the narrow bandwidths of nanoshots of the GPs from the main pulse of the Crab pulsar, observed at frequencies of $6-10 \mathrm{GHz}$ (Hankins \& Eilek 2007). According to Hankins et al. (2003), the coherent radio emission in main pulse GPs from the Crab pulsar is plasma emission produced by collapsing solitons in SPT. The SPT model also predicts that plasma flow in the radio emission region is likely to be unsteady, and the amount of energy released from one patch to another can differ. This could explain occasional narrow-band pulses at low frequencies for B0809+74. If true, this would also provide a direct link between giant pulses and anomalously intensive pulses observed at low frequencies.

\subsection{Summary}

We presented an analysis of the pulse spectra and pulse energy and peak flux density distributions for the pulsars B0809+74 and B1133+16. Here we summarize our findings:

- The energy of strong pulses are not large enough to be called giant pulses in the conventional way, i.e. energies $>10\langle E\rangle$.

- The fits of lognormal and power-law distributions to the energies of the pulses do not allow us to differentiate between them as the significance of the fits is still low. However, in general, a lognormal distribution fits slightly better, but power-law distributions work better for the high-energy distribution tails.

- There are no apparent breaks in both pulse energy and peak flux density distributions, but there is an intrinsic break in peak flux density distributions at about $12\langle S\rangle$ and $3\langle S\rangle$ for B0809+74 and B1133+16, respectively. This might indicate the presence of two pulse populations. 
- Stronger pulses from B0809+74 are narrower and tend to come in the inner part of the average profile. In the case of B0809+74, more than $50 \%$ of the pulses stronger than $20 \sigma$ come in the second component.

- Some of the pulses from B0809+74 show very narrow-band spectra especially at very low frequencies below $30 \mathrm{MHz}$, with some of the emission patches being as narrow as $1 \mathrm{MHz}$. The emission from B1133+16 is much broader.

- For B0809+74 strong emission patches tend to have a width of $3 \mathrm{MHz}$, but range from 1 up to $16 \mathrm{MHz}$.

- The narrow-band origin of the pulses from B0809+74 appears to be intrinsic to the pulsar rather than being due to propagation effects in the ISM or ionosphere.

- The spectral width of strong emission patches with energies $E>2\langle E\rangle$ scales with increasing frequency as $\Delta f / f \sim 0.15$ in the case of B0809+74. This can be understood in the context of the strong plasma turbulence model (Weatherall 1998), as in the case of GP nanoshots from the main pulse of the Crab pulsar at frequencies of 6-10 GHz. This would support a relation between anomalously intensive pulses at low frequencies and giant pulses. 

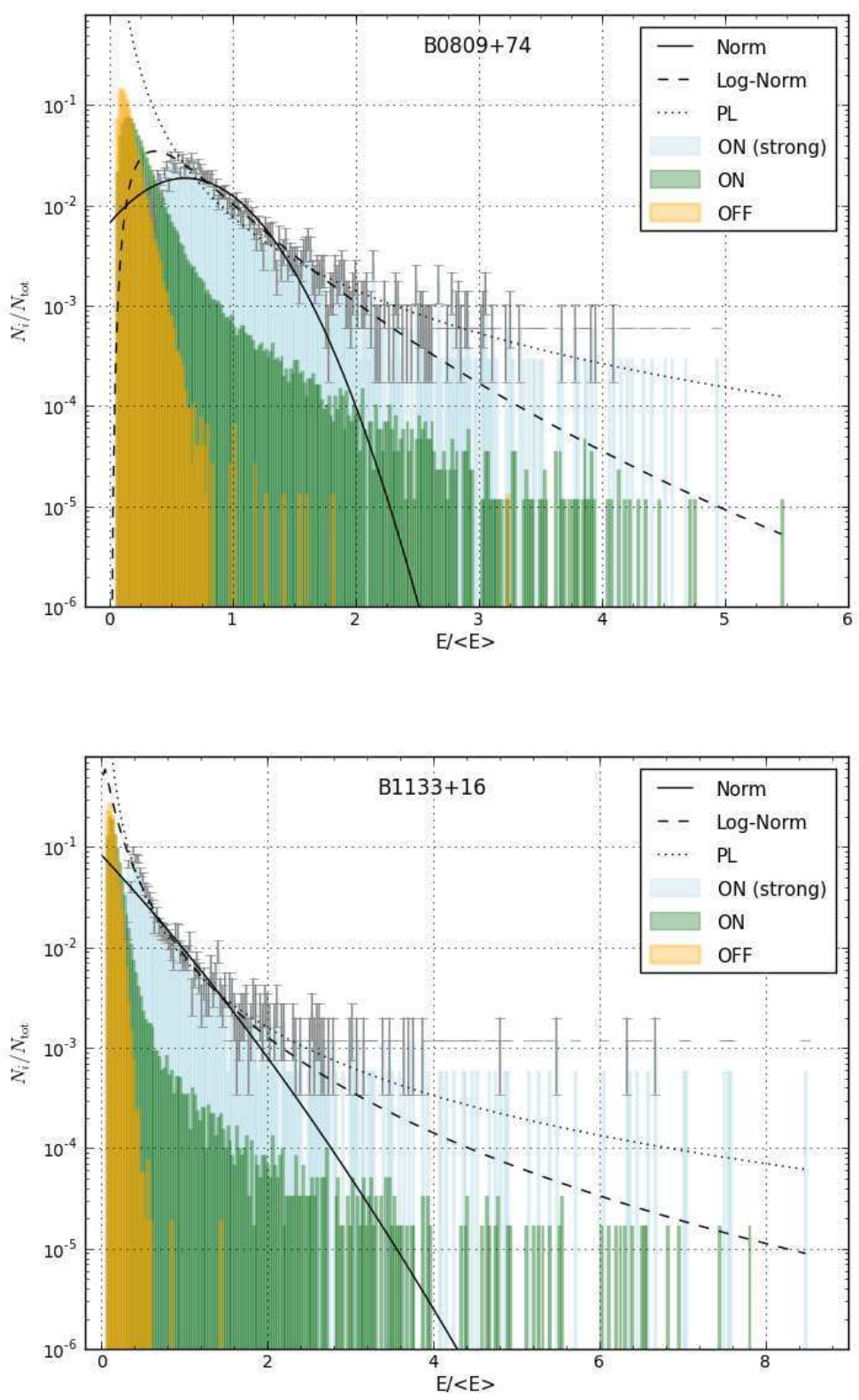

Fig. 5.2.- Pulse energy distributions for the pulsars B0809+74 (top) and B1133+16 (bottom). Energies are for individual emission patches over the patch's frequency width normalized by the energy of the average profile over the entire band. Distributions in green and yellow are for ON-pulse and OFF-pulse phase windows respectively, using the same search technique with a threshold of $1 \sigma$. The distributions in light blue are for strong patches with the peak flux density over $5 \sigma$ on the spectrum averaged over the patch phase window. Best fits for normal, log-normal and power-law distributions are shown by solid, dashed and dotted lines, respectively 

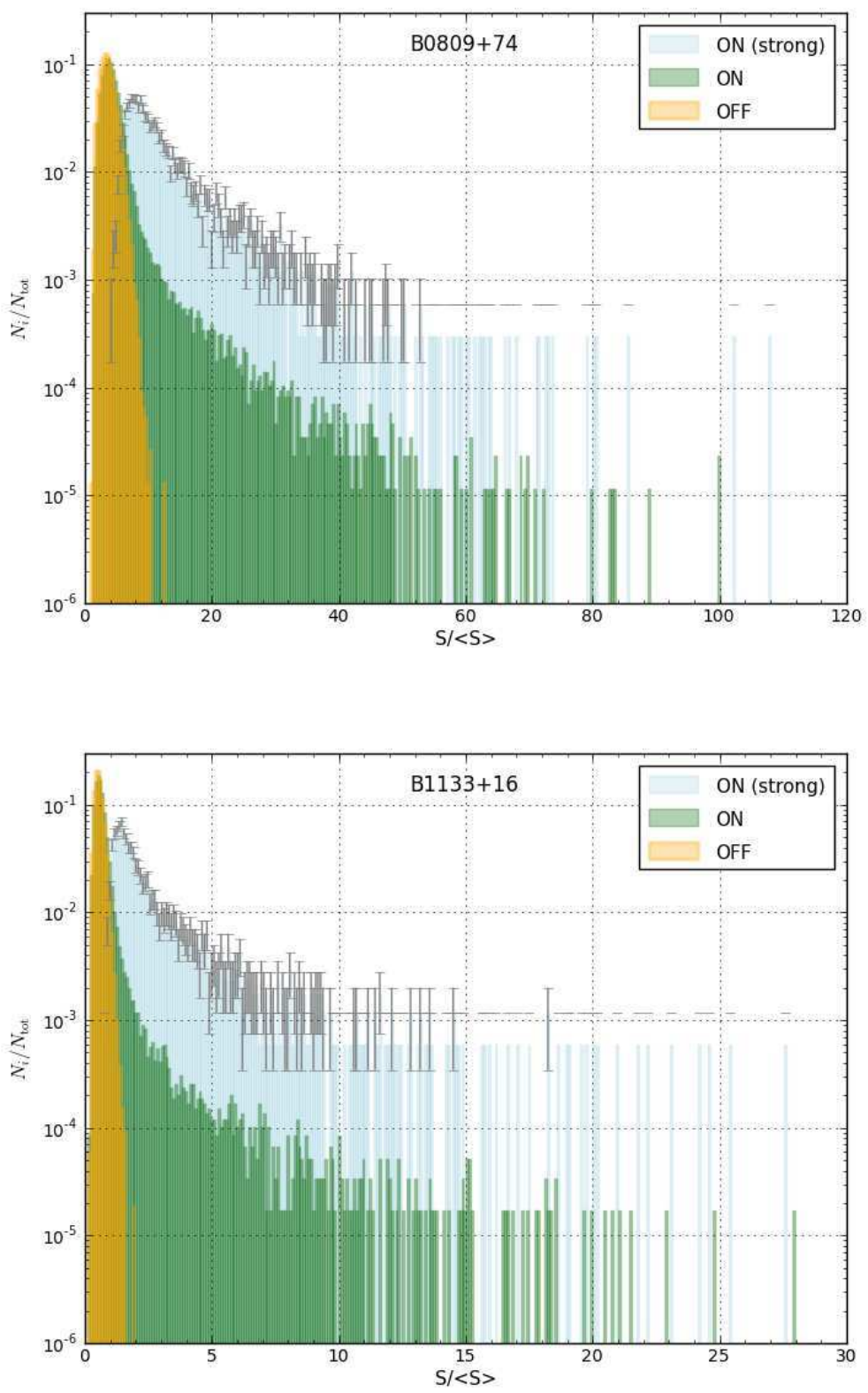

Fig. 5.3.- Pulse peak flux density distributions for B0809+74 (top) and B1133+16 (bottom). Peak flux densities are for the emission patches normalized by the peak flux density of the average profile over the entire band. Distributions are similar to Figure 5.2. 
Table 5.2: Best-fit parameters to the pulse energy distributions for the pulsars B0809+74 and B1133+16

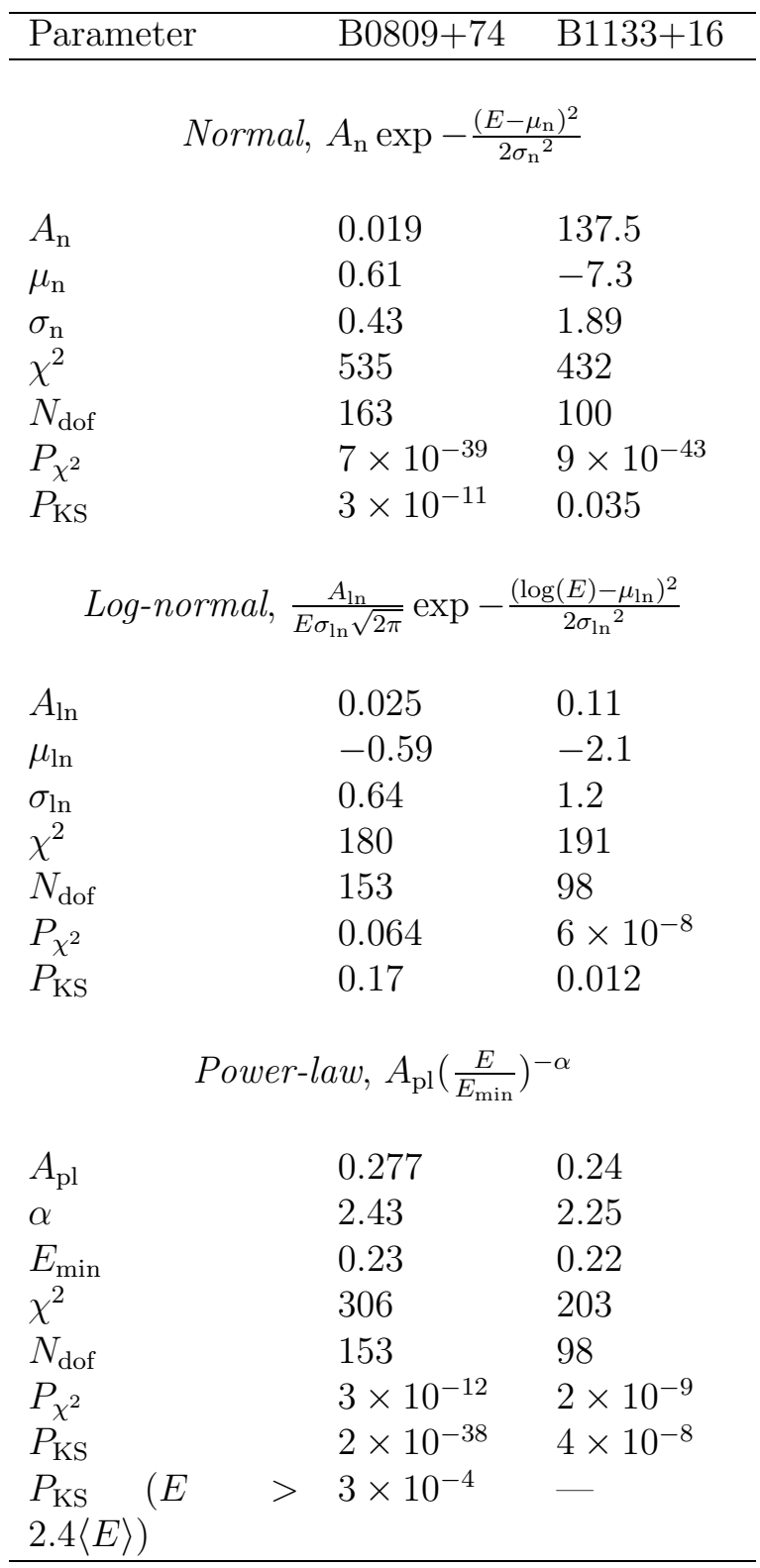



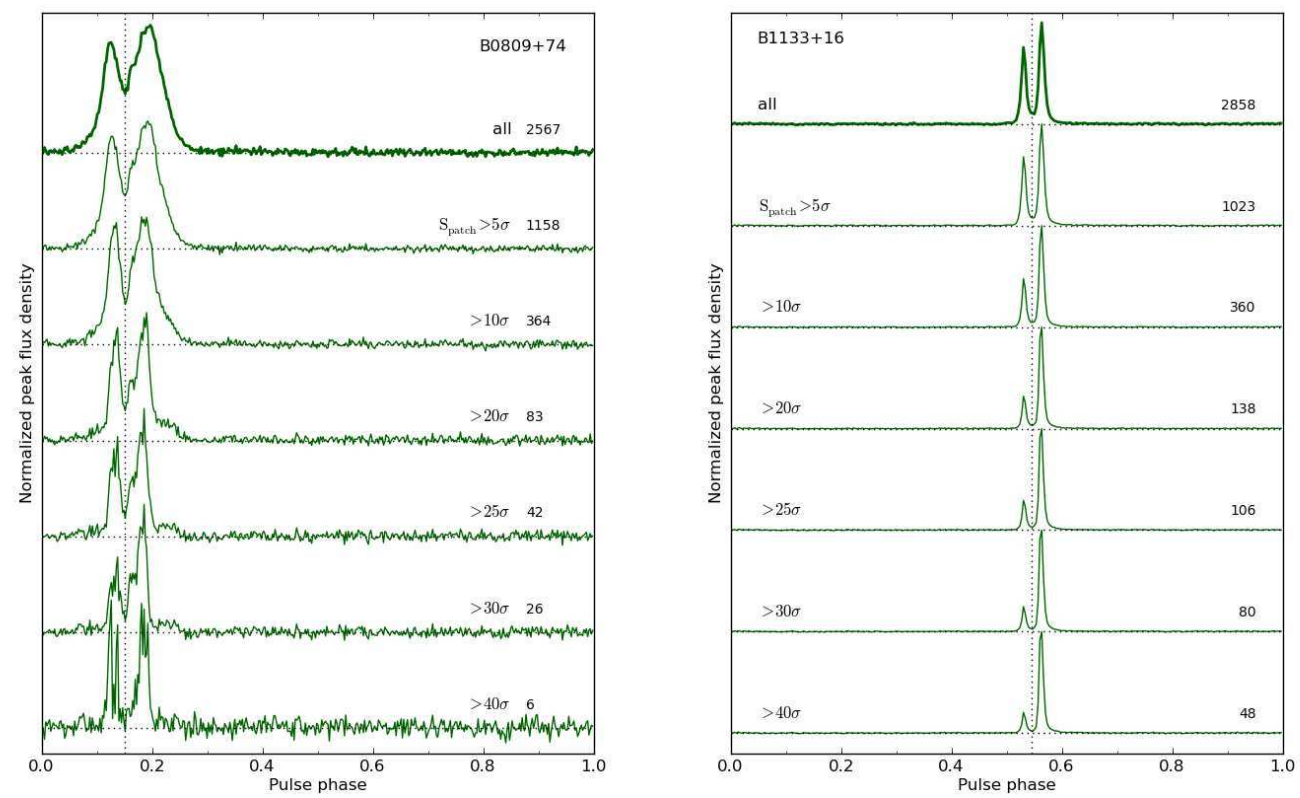

Fig. 5.4.- Average pulse profiles for B0809+74 (left) and B1133+16 (right). The profiles on top of the panels are comprised of all the pulse periods from the observations. Then, from top to bottom, profiles are shown that are comprised only of pulses with peak flux density larger than $5,10,20,25,30$, and $40 \sigma$, respectively. Each profile is normalized by its maximum. The number of periods included for corresponding profiles of pulsar B0809+74 are 2567 (all), 1158, 364, 83, 42, 26 and 6 (from top to bottom), and for B1133+16 - 2858 (all), 1023, 360, 138, 106, 80, and 48. 

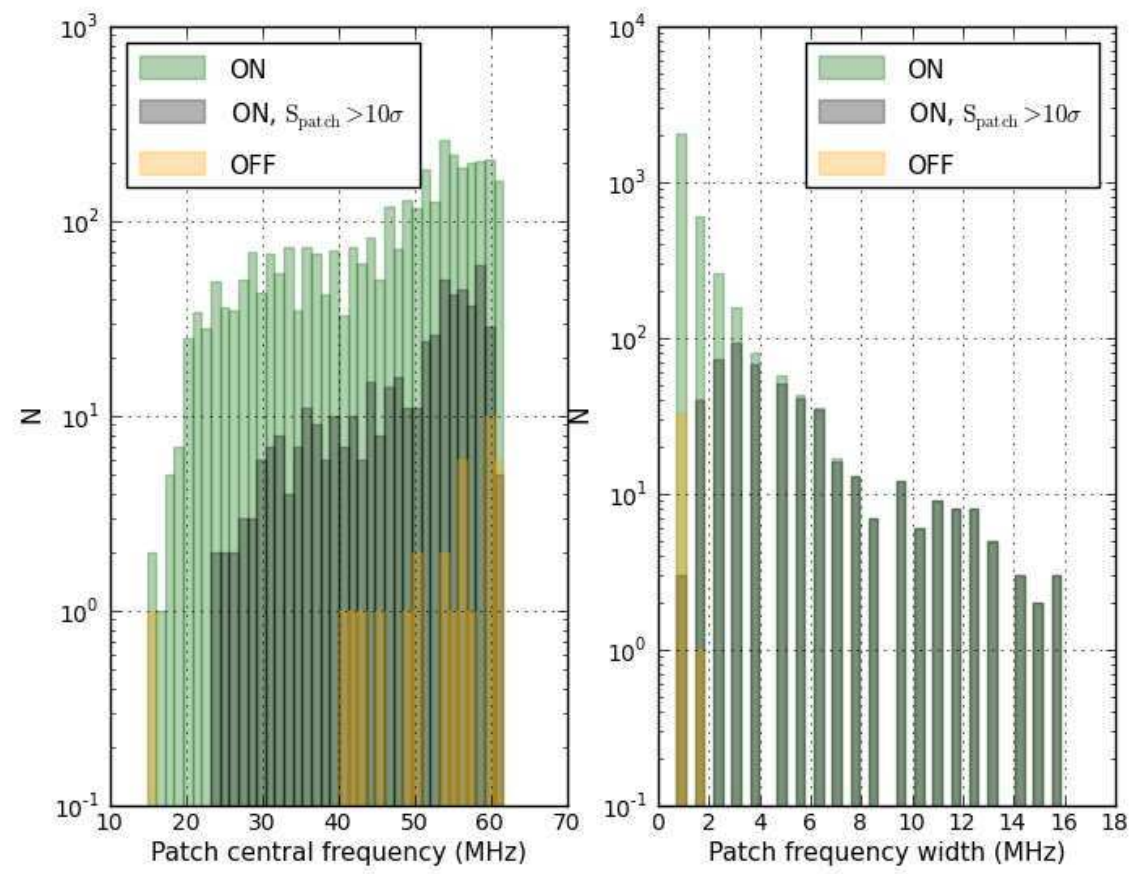

Fig. 5.5.- Histograms of patch central frequency (left) and their frequency width (right) for the ON-pulse window (green), patches with peak flux density stronger than $10 \sigma$ (black), and OFF-pulse window (yellow) for the pulsar B0809+74. For both ONpulse and OFF-pulse histograms only patches with spectral peak flux density stronger than $5 \sigma$ are used according to our pulse selection pipeline. 

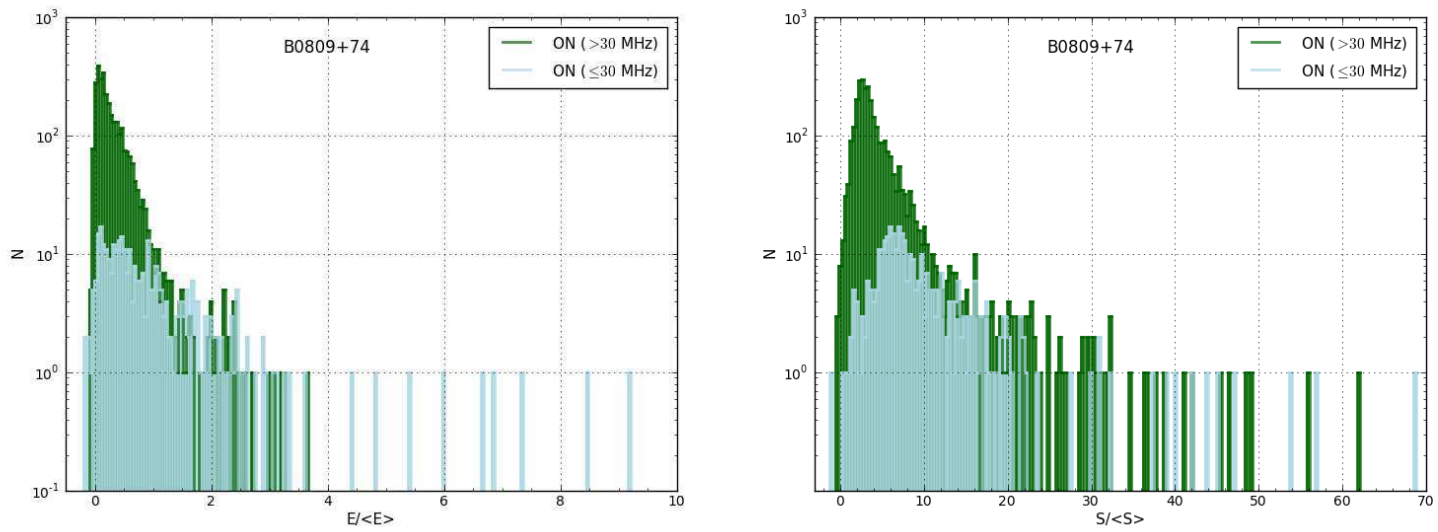

Fig. 5.6.- Pulse energy (left) and peak flux density (right) distributions for the B0809+74 shown separately for pulses with the emission patch below $30 \mathrm{MHz}$ (light blue) and above $30 \mathrm{MHz}$ (dark green).
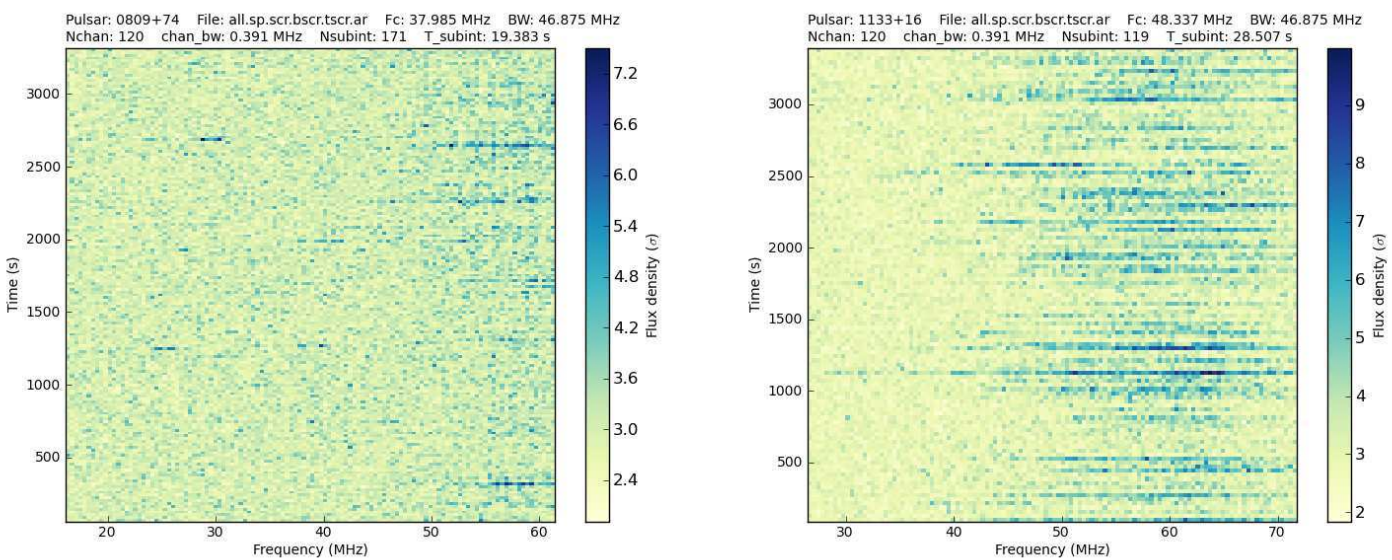

Fig. 5.7.- Dynamic spectra of pulsars B0809+74 (left) and B1133+16 (right) over the entire frequency band. Each spectrum is decimated in time and frequency to provide a frequency resolution of about $391 \mathrm{kHz}$ and time resolution of about $19 \mathrm{~s}$ for $\mathrm{B} 0809+74$ and $28.5 \mathrm{~s}$ for B1133+16. 


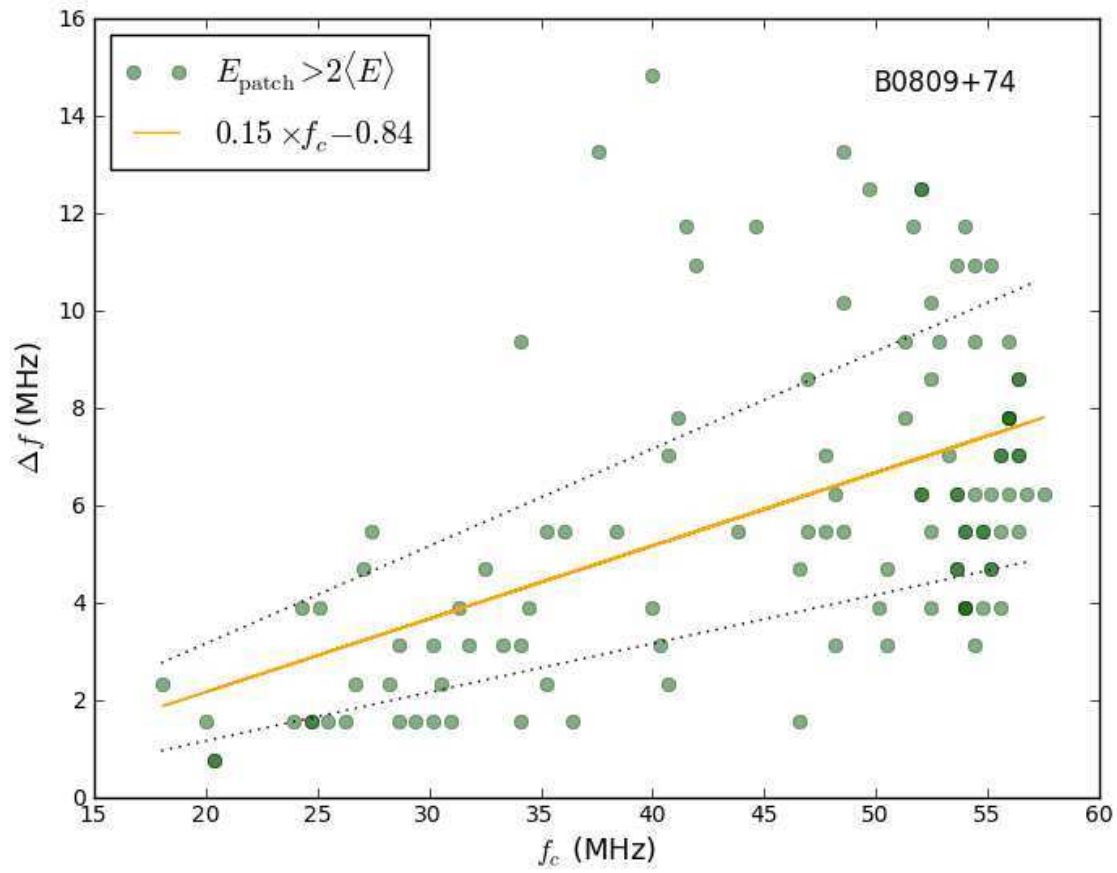

Fig. 5.8.- Dependence of emission patch spectral width on emission frequency (central frequency of the patch) for the all strong emission patches from B0809+74 with energy $E>2\langle E\rangle$ (green circles). The orange line, $\Delta f / f_{\mathrm{c}} \sim 0.15$, shows the best least-squares fit. The dotted lines show the same dependency but for slopes of 0.1 and 0.2 . 


\section{Chapter 6}

\section{Terzan5A: Timing and Single Pulses}




\subsection{Introduction}

Ter5A is the second eclipsing binary system discovered (Lyne et al. 1990). The pulsar inhabits a tight $1.7 \mathrm{hr}$ circular orbit with $\mathrm{a} \sim 0.1 \mathrm{M}_{\text {sol }}$ companion star, presumably a low-mass main sequence star or a white dwarf (Nice \& Thorsett 1992).

The companion overfills its Roche lobe and the escaping plasma produces variable irregular eclipses. Usually the pulsar is eclipsed for about one third of the orbit, around the point of inferior conjunction. However, small clouds of unbound plasma can cause "eclipses" at any orbital phase. Sometimes the pulsar is even completely enshrouded for several orbital periods. The pulsed signal near eclipse edges is strongly attenuated and there are observed excess pulse delays. A thorough analysis of possible eclipse mechanisms was conducted by Thompson et al. (1994). According to their work, most probably Ter5A is eclipsed when the radio waves scatter off plasma turbulence generated by the intense radio beam via Raman scattering, although other eclipse mechanisms are still possible. Modeling eclipses in the Ter5A binary system is impeded by the lack of a known intrinsic pulsar spin-down power. The observed $\dot{P}$ is negative and is completely biased by acceleration in the field of the globular cluster and the Galactic center (Nice \& Thorsett 1992).

Tight interaction between the pulsar, binary companion and unbound plasma makes binary evolution interesting and somewhat hard to predict. After a decade of timing, Nice et al. (2000) reported the secular decrease in orbital period of the system, leading to a evolutionary timescale of $200 \mathrm{Myr}$, about an order of magnitude smaller than the orbital decay scale due to gravitational

radiation, $\left|P_{b} / \dot{P}_{b}\right|_{\mathrm{GR}}=1000 \mathrm{Myr}$. This rapid decay was interpreted by Ergma \& Sarna (2000) as an evidence of magnetic braking.

However, extending the timing dataset to two decades shows that the previously reported secular decrease of the orbital period is only a part of long-term quasiperiodical modulation. We report this result in the first half of this chapter. 
The second half contains single-pulse analysis of Ter5A radio emission.

\subsection{Timing}

Timing observations were carried out over the past 22 years using four different telescopes and several pulsar backends (see Table 6.1). The data prior to 1999 were published in Nice et al. (2000).

For the analysis of pulse times of arrival we used tempo2 in tempo emulation mode. We used the BT binary model, setting the orbital eccentricity to 0 (Nice $\&$ Thorsett 1992). Pulsar coordinates were fixed at the interferometric position reported by Fruchter \& Goss (2000).

Using the timing solution from Nice et al. (2000) as a starting point, we performed separate fits of spin and orbital parameters using small overlapping subsets of data. The procedure was as follows:

- divide 22 years into chunks of 400 days with 200 days overlap. If the time span between the first and the last TOA in the chunk is bigger than 100 days, fit spin frequency $F_{0}$ and its derivative $F_{1}$, setting the $F_{0}$ epoch to the middle of observation. If the timespan is smaller than 100 days, fit $F_{0}$ only. Together with the spin parameters, fit the projected semimajor axis $A_{1}$ and the epoch of the periastron passage $T_{0}$ (defined as the time of the ascending node for orbits with exactly eccentricity $=0$ in the BT model). Fit dispersion measure DM if the data has more than one observing frequency. After 1999, if the data included 2 or more telescopes or backends, fit for a constant instrumental offset between them.

- within each chunk, fix $F_{0}$ and $F_{1}$ and repeat fits of orbital parameters and dispersion measure (if possible) for 10-day subsets of data with overlaps of 5 days if TOAs cover more than 0.8 of a full orbit in a subset.

Figure 6.1 shows the observed change of the pulsar spin period, $P$, projected semi-major axis, $A_{1}$, dispersion measure, DM, and the epoch of passage of 

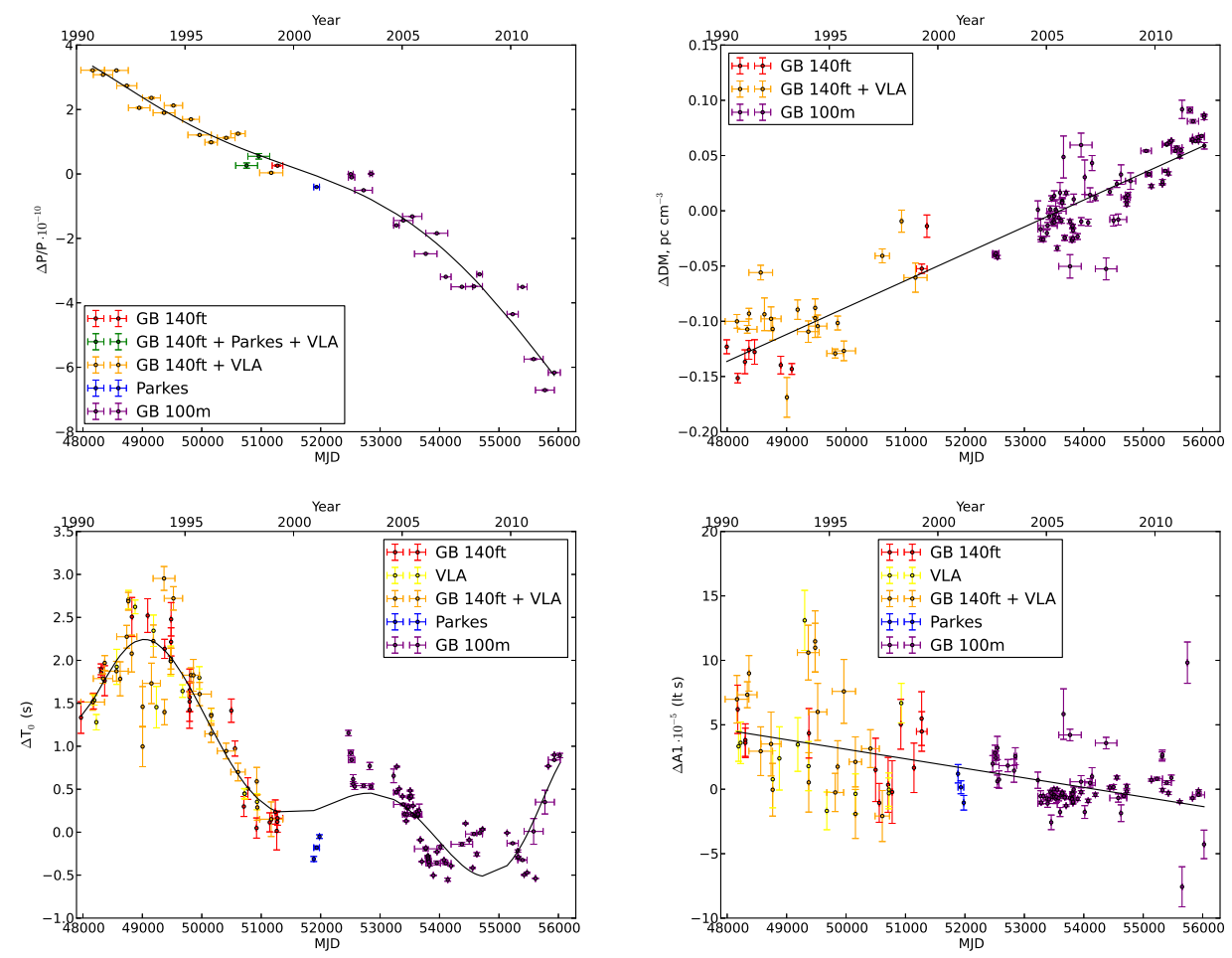

Fig. 6.1. - Evolution of spin period (top left), dispersion measure (top right), epoch of the periastron passage (basically the antiderivative of the orbital period, bottom left) and the projected semimajor axis (bottom right). Errorbars on the $\mathrm{X}$-axis represent the time span of each measurement. Errorbars on the Y-axis are tempo errors. Black line shows polynomial fits.

periastron, $T_{0}$. The latter is equivalent to a fixed value of $T_{0}$ and a variable orbital period $P_{b}$. The derived characteristic rate of change of the orbital period $\dot{P}_{b} / P_{b}$ is about $\pm 3 \times 10^{-15} \mathrm{~s}^{-1}$. Similarly, the derived rate of change of the pulsar spin period $\dot{P} / P \sim-2 \times 10^{-18} \mathrm{~s}^{-1}$, the $\dot{A}_{1} / A_{1} \approx-8.6 \times 10^{-14} \mathrm{~s}^{-1}$, and $\dot{\mathrm{DM}} \approx 8 \times 10^{-3} \mathrm{pc} \mathrm{cm}^{-3} \mathrm{yr}^{-1}$. The negative sign of $\dot{P} / P$ can be caused by the acceleration in the globular cluster, Galactic gravitational potential, acceleration due to encounter with another star or proper motion (Shklovskii effect). From Nice \& Thorsett (1992), the contribution from the acceleration in the globular cluster and Galactic potential is about $\approx 2 \times 10^{-17} \mathrm{~s}^{-1}$ and $\sim 3 \times 10^{-18} \mathrm{~s}^{-1}$, respectively. Both values are comparable to intrinsic $\dot{P} / P$ of 
pulsars with similar periods (which ranges from $\sim 10^{-18}$ to $\sim 10^{-16}$ ). As was shown by Phinney (1992), observing the effect of close encounter with another star in a globular cluster highly unlikely, although still possible. For any feasible pulsar relative velocity (less than couple of hundreds $\mathrm{km} / \mathrm{s}$ ) Shklovskii effect will contribute less than $10^{-18} \mathrm{~s}^{-1}$.

The observed variation of the orbital period is about 1000 times larger than that for the spin period and shows quasi-periodic modulation. Though acceleration in the globular cluster, Galactic potential, and Shklovskii effect can contribute significantly to the variation of the spin period, they are still very small to account for observed rate of change of the orbital period. A third body (or bodies) in the system would cause an effect of the same magnitude on both $\dot{P} / P$ and $\dot{P}_{b} / P_{b}$, which is not observed. Lazaridis et al. (2011) explained periodic changes in the orbital period of PSR J2051-0827 by tidal dissipation leading to changes in the gravitational quadrupole moment of the companion. Unfortunately, Ter5A belongs to a highly reddened globular cluster and lies between two bright giant stars, which makes it hard to detect the variability of the companion that is needed for the model (Edmonds et al. 2001).

A discussion of binary parameter evolution would not be complete without mentioning that the observed rate of change of the projected semi-major axis can be explained by mass loss from the binary system.

By the Kepler's third law, ${\dot{A_{1}}}^{m} / A_{1}=2 / 3 \dot{P}_{b}^{m} / P_{b}$. Assuming a circular orbit, no mass loss from the pulsar $\left(\dot{m}_{p}=0\right)$, and a rate of mass loss from the companion $\dot{m}_{c}>0$,

$$
\dot{m}_{c}=-\frac{M}{2} \frac{\dot{P}_{b}}{P_{b}} \simeq 7.1 \times 10^{-9} M_{\odot} \mathrm{y} r_{-1},
$$

for $\dot{P}_{b} / b=3 \times 10^{-h 5} \mathrm{~s}^{-1}$. Here $M$ is the total mass of the system. The value of $\dot{m}_{c}$, obtained this way, agrees well with both estimate from the Nice \& Thorsett (1992) and with what is predicted by ablation models. However, mass loss cannot explain periodic variation of $P_{b}$.

Finally, the emission of gravitational waves contributes $-2.7 \times 10^{-17} \mathrm{~s}^{-1}$ and 
might be responsible for the long-term secular decrease of the orbital period.

\subsection{Single pulses}

For our single-pulse study, we selected three observing sessions, at $820 \mathrm{MHz}$, L- and S-bands. Each session contained four full orbits and 512 frequency channels. Besides creating de-dispersed time series, we also folded the data at the pulsar ephemeris into 512 phase bins with 60-second subintegrations. Data were calibrated and the single pulses were extracted in the same way as for B1821-24A, Section 4.2.

The total intensity of both the average pulse profile and detected single-pulses versus orbital and spin phases is plotted on Figures 6.3, 6.4, 6.5, upper subplot, top. The pulsar is usually eclipsed around orbital phase 0.25 , the point of inferior conjunction. Close to ingress and egress pulses are delayed due to increasing DM. Sometimes the pulsar is also eclipsed at other orbital phases (Fig. 6.5).

About $20 \%$ of Ter5A's radio flux is circularly polarized. The radio flux retains its circular polarization even when it is attenuated and scattered at the edges of eclipses (same Figures, the middle of upper subplots). On the contrary, the fraction of linear polarization seems to drop before any signs of eclipse influence can be spotted. The lack of linear polarization could be explained by large changes in the Rotation Measure (RM) around eclipses. We searched for a maximum in the fractional linear polarization in the on-pulse phase window by correcting the archives for Faraday rotation with a set of trial RMs from -256 to $512 \mathrm{rad} / \mathrm{m}^{2}$. For all $m_{l}$ with $\mathrm{S} / \mathrm{N}>4$ the average $\mathrm{RM}$ was $190 \mathrm{rad} / \mathrm{m}^{2}$ and the standard deviation was $10 \mathrm{rad} / \mathrm{m}^{2}$.

We detect single pulses from Ter5A in all on-pulse windows throughout all orbital phases, except within the dispersed tails on eclipse edges. However right when the pulsar is about to go in or out of eclipse, there appear bursts of pulses, with widths of the on-pulse window and energies within 12 times energy 


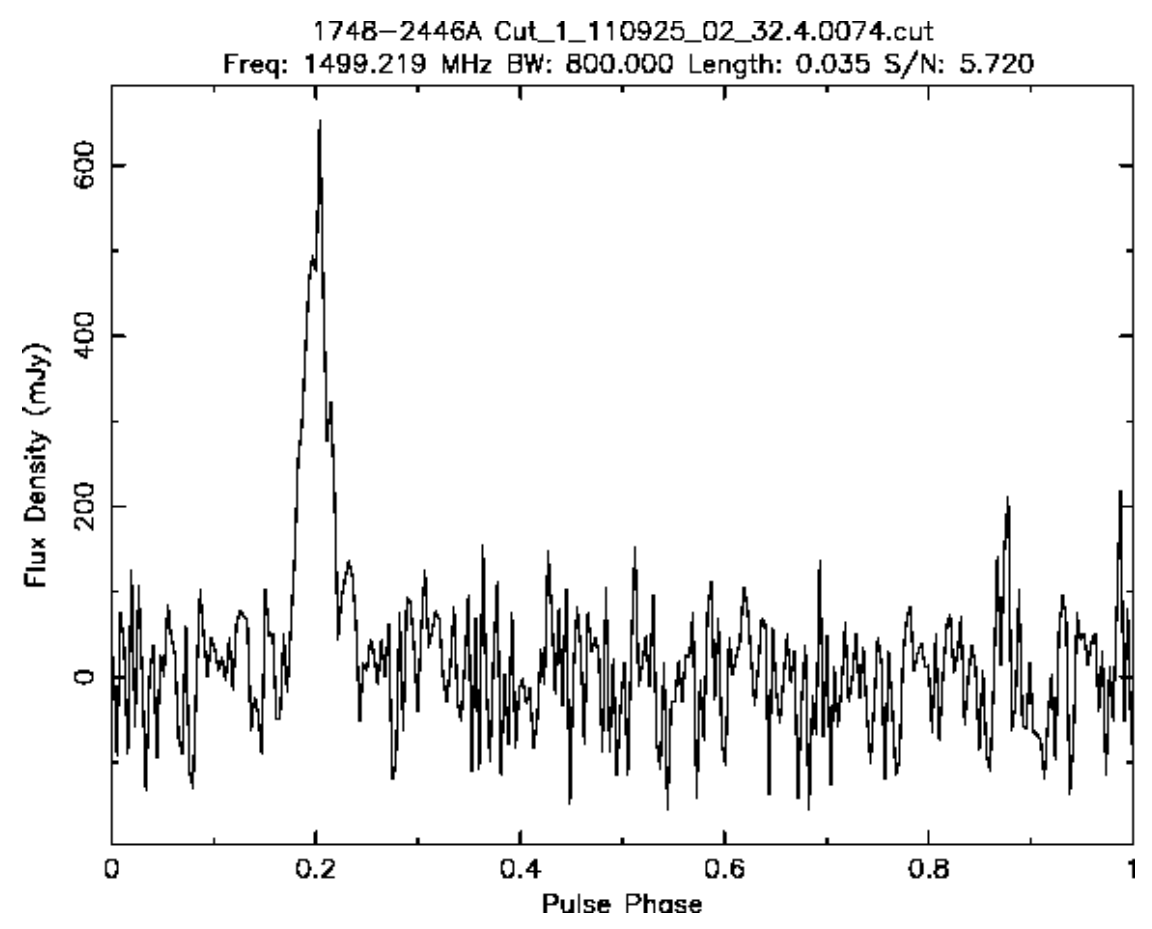

Fig. 6.2.- An example of strong single pulse in L-band. Pulse does not have any noticeable scattering tail and is about as wide as the average profile. The energy of the pulse is 8 times the energy of the average profile.

of the uneclipsed integrated pulse profile. Although they formally can be called "Giant" due to their energies (Kuzmin 2006), these pulses are broad and occur uniformly within an on-pulse phase window.

Bursts of single pulses appear exclusively in the vicinity of eclipses, which leads to hypothesis that these pulses are a product of an interaction between the pulsar and companion material. It is unlikely that this interaction is happening inside pulsar's magnetosphere, because magnetosphere is very small comparing to the size of the system - the radius of the light cylinder is about $7 \cdot 10^{-4} R_{\odot}$, whereas binary separation is $0.85 R_{\odot}$ and the size of Roche lobe is $0.05 R_{\odot}$.

Strong pulses come at a rate of about 1 per second in bunches typically $1-5$ minutes long. During these bursts the average (integrated over 1 minute) pulsar signal is not scattered or attenuated/amplified (Figures 6.3, 6.4, 6.5, lower subplot) 
and single pulses themselves do not have visible scattering tails (see Fig. 6.2). If the timescale of diffractive scintillation due to multi-path propagation in intra-binary material is very small, the scintillation will amplify some single pulses while leaving average emission unaffected. For scattering timescales less than our time resolution, a scintillation time of 1 second requires the relative velocity of the diffractive screen to be about $4000 \mathrm{~km} / \mathrm{s}$. Most of this velocity should come from intra-binary material since both pulsar's and Earth's orbital velocities are at least two orders of magnitude smaller.

Normal single pulses can also be amplified by refraction on small, very sparse clumps of plasma. This hypothesis does not require such high velocity of the screen.

\subsection{Summary}

The orbital period of the Ter5A binary system undergoes quasi-periodic oscillations with $\dot{P}_{b} / P_{b}$ three orders of magnitude larger than any fractional changes in spin period, $\dot{P} / P$. It can be attributed to tidal dissipation leading to changes in the quadrupole moment of the companion, however more information about the companion is needed before making conclusions.

Ter5A has a substantial degree of the circular polarization, which is preserved throughout the eclipse and even within the scattered tails of eclipse ingress and egress. On the contrary, linear polarization tends to disappear before any signs of eclipse influence can be spotted.

Ter5A emits strong broad pulses, with energies up to $\sim 10$ times bigger than the energy of the integrated uneclipsed profile. These pulses have about the same width as the on-pulse window and appear in bursts when the pulsar is about to go in or out of eclipse. Strong single pulses may be the normal single pulses amplified by the refraction on small very sparse clumps of intra-binary plasma. 
Table 6.1. Observation Summary

\begin{tabular}{lcc}
\hline \multicolumn{1}{c}{ Telescope } & Epoch & Frequency, MHz \\
& & \\
\hline Green Bank 140 ft & $1990-1999$ & 1330,1660 \\
VLA & $1990-1999$ & 1660 \\
Parkes & $1998-2001$ & 1360 \\
GBT & $2002-2012$ & 820,1400, \\
& & $1100-1900$, \\
& & $1600-2400$ \\
\hline
\end{tabular}



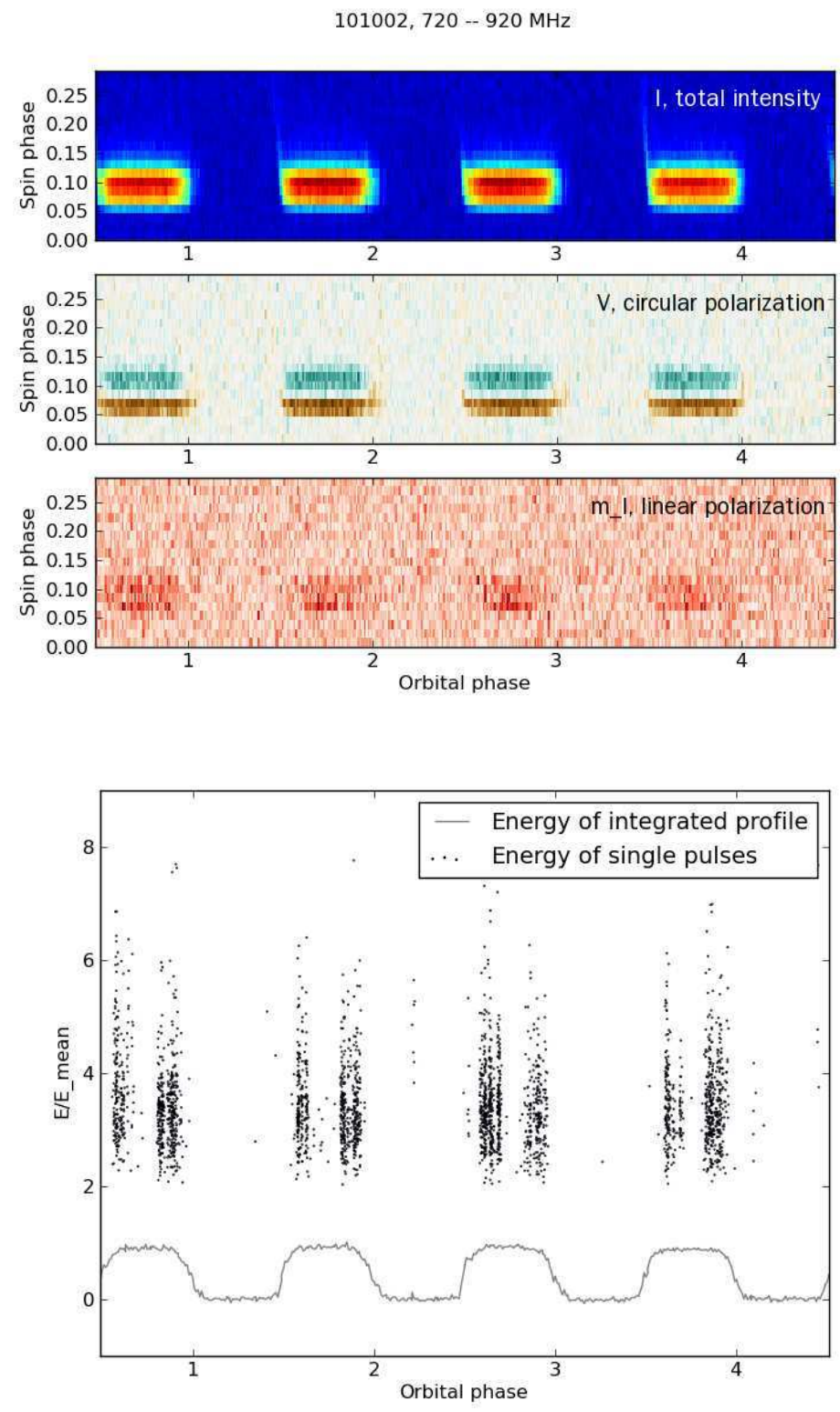

Fig. 6.3.- Upper subplot, top: total intensity $I$ at $720-920 \mathrm{MHz}$, plotted versus spin and orbital phase. Pulsar is eclipsed around the point of inferior conjunction (orbital phase $\left.\phi_{b}=0.25\right)$. Weak dispersive delays are seen at $\phi_{b} 1.5$ and 3.5. Middle: circular polarization $V$ (brown - negative, green - positive). Note that circular polarization is present throughout all uneclipsed orbital phases. Bottom: Linear polarization $m_{l}$. Note that the radio signal is linearly polarized only through part of the orbit. Bottom subplot: energy of single pulses versus orbital phase, normalized by the average energy of the uneclipsed integrated profile. The energy of the integrated profile versus orbital phase is overplotted for reference. 
$110925,1100-1900 \mathrm{MHz}$
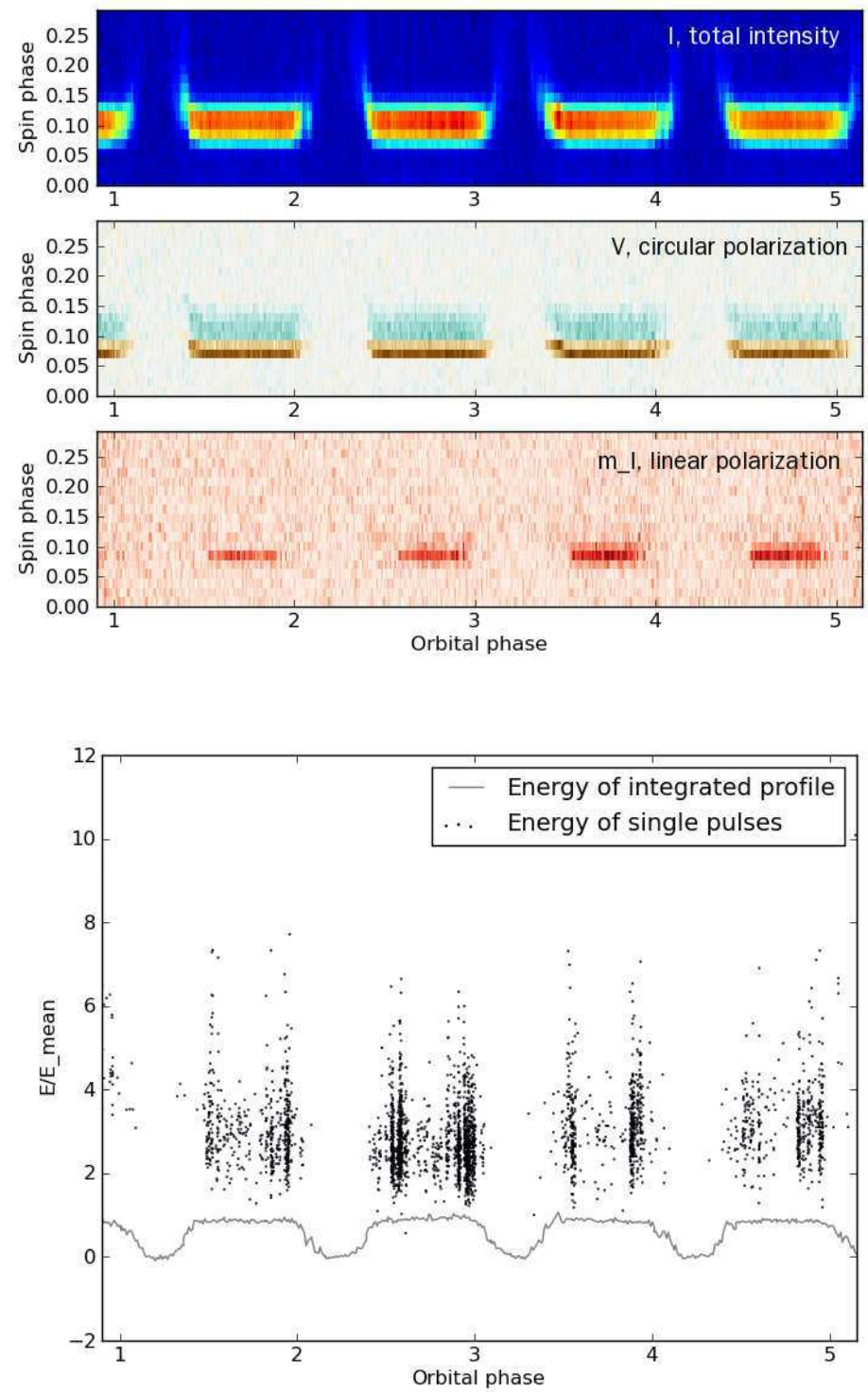

Fig. 6.4.- Upper subplot, top: total intensity $I$ at $1100-1900 \mathrm{MHz}$, plotted versus spin and orbital phase. Pulsar is eclipsed around the point of inferior conjunction (orbital phase $\phi_{b}=0.25$ ). Strong dispersive delays are seen at every ingress and egress. Middle: circular polarization $V$ (brown - negative, green - positive). Note that circular polarization is present throughout all uneclipsed orbital phases. Bottom: Linear polarization $m_{l}$. Note that the radio signal is linearly polarized only through part of the orbit. Bottom subplot: energy of single pulses versus orbital phase, normalized by the average energy of the uneclipsed integrated profile. The energy of the integrated profile versus orbital phase is overplotted for reference. 
$100815,1600-2400 \mathrm{MHz}$
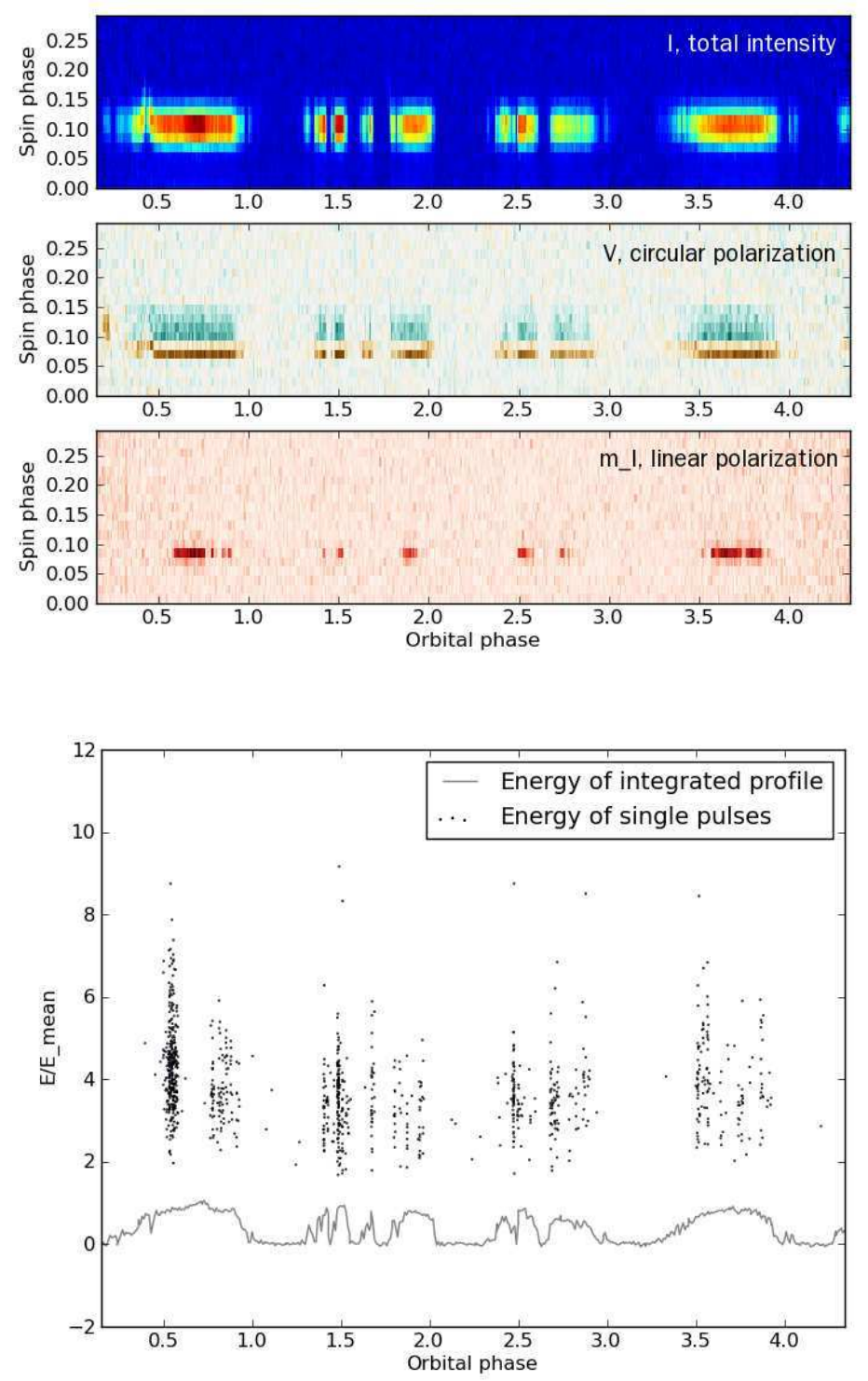

Fig. 6.5.- Upper subplot, top: total intensity $I$ at $1600-2400 \mathrm{MHz}$, plotted versus spin and orbital phase. Pulsar is eclipsed around the point of inferior conjunction (orbital phase $\phi_{b}=0.25$ ). Several mini-eclipses are seen at other $\phi_{b}$ as well. Middle: circular polarization $V$ (brown - negative, green - positive). Note that circular polarization is present throughout all uneclipsed orbital phases. Bottom: Linear polarization $m_{l}$. Note that the radio signal is linearly polarized only through part of the orbit. Bottom subplot: energy of single pulses versus orbital phase, normalized by the average energy of the uneclipsed integrated profile. The energy of the integrated profile versus orbital phase is overplotted for reference. 


\section{References}

Abdo, A. A., Ackermann, M., Ajello, M., Atwood, W. B., Axelsson, M., et al. 2010, ApJ, 708, 1254

Aliu, E., Anderhub, H., Antonelli, L. A., Antoranz, P., Backes, M., Baixeras, C., Barrio, J. A., Bartko, H., Bastieri, D., Becker, J. K., Bednarek, W., Berger, K., Bernardini, E., Bigongiari, C., Biland, A., Bock, R. K., Bonnoli, G., Bordas, P., Bosch-Ramon, V., Bretz, T., Britvitch, I., Camara, M., Carmona, E., Chilingarian, A., Commichau, S., Contreras, J. L., Cortina, J., Costado, M. T., Covino, S., Curtef, V., Dazzi, F., De Angelis, A., De Cea del Pozo, E., de los Reyes, R., De Lotto, B., De Maria, M., De Sabata, F., Delgado Mendez, C., Dominguez, A., Dorner, D., Doro, M., Elsässer, D., Errando, M., Fagiolini, M., Ferenc, D., Fernandez, E., Firpo, R., Fonseca, M. V., Font, L., Galante, N., Garcia Lopez, R. J., Garczarczyk, M., Gaug, M., Goebel, F., Hadasch, D., Hayashida, M., Herrero, A., Höhne, D., Hose, J., Hsu, C. C., Huber, S., Jogler, T., Kranich, D., La Barbera, A., Laille, A., Leonardo, E., Lindfors, E., Lombardi, S., Longo, F., Lopez, M., Lorenz, E., Majumdar, P., Maneva, G., Mankuzhiyil, N., Mannheim, K., Maraschi, L., Mariotti, M., Martinez, M., Mazin, D., Meucci, M., Meyer, M., Miranda, J. M., Mirzoyan, R., Moles, M., Moralejo, A., Nieto, D., Nilsson, K., Ninkovic, J., Otte, N., Oya, I., Paoletti, R., Paredes, J. M., Pasanen, M., Pascoli, D., Pauss, F., Pegna, R. G., PerezTorres, M. A., Persic, M., Peruzzo, L., Piccioli, A., Prada, F., Prandini, E., Puchades, N., Raymers, A., Rhode, W., Ribó, M., Rico, J., Rissi, M., Robert, A., Rügamer, S., Saggion, A., Saito, T. Y., Salvati, M., Sanchez- 
Conde, M., Sartori, P., Satalecka, K., Scalzotto, V., Scapin, V., Schweizer, T., Shayduk, M., Shinozaki, K., Shore, S. N., Sidro, N., Sierpowska-Bartosik, A., Sillanpää, A., Sobczynska, D., Spanier, F., Stamerra, A., Stark, L. S., Takalo, L., Tavecchio, F., Temnikov, P., Tescaro, D., Teshima, M., Tluczykont, M., Torres, D. F., Turini, N., Vankov, H., Venturini, A., Vitale, V., Wagner, R. M., Wittek, W., Zabalza, V., Zandanel, F., Zanin, R., Zapatero, J., de Jager, O. C., de Ona Wilhelmi, E., \& MAGIC Collaboration. 2008, Science, 322,1221

Backer, D. C., \& Sallmen, S. T. 1997, The Astronomical Journal, 114, 1539

Bhat, N. D. R., Cordes, J. M., Camilo, F., Nice, D. J., \& Lorimer, D. R. 2004, ApJ, 605, 759

Bilous, A. V., Kondratiev, V. I., McLaughlin, M. A., Ransom, S. M., Lyutikov, M., Mickaliger, M., \& Langston, G. I. 2011, ApJ, 728, 110

Bogdanov, S., van den Berg, M., Servillat, M., Heinke, C. O., Grindlay, J. E., Stairs, I. H., Ransom, S. M., Freire, P. C. C., Bégin, S., \& Becker, W. 2011, ApJ, 730, 81

Burke-Spolaor, S., Johnston, S., Bailes, M., Bates, S. D., Bhat, N. D. R., Burgay, M., Champion, D. J., D’Amico, N., Keith, M. J., Kramer, M., Levin, L., Milia, S., Possenti, A., Stappers, B., \& van Straten, W. 2012, MNRAS, 423, 1351

Cairns, I. H. 2004, ApJ, 610, 948

Chung, C. T. Y., \& Melatos, A. 2011, MNRAS, 415, 1703

Cognard, I., Shrauner, J. A., Taylor, J. H., \& Thorsett, S. E. 1996, ApJ, 457, $\mathrm{L} 81+$

Cordes, J. M., Bhat, N. D. R., Hankins, T. H., McLaughlin, M. A., \& Kern, J. 2004, ApJ, 612, 375 
Crossley, J. H., Eilek, J. A., Hankins, T. H., \& Kern, J. S. 2010, ApJ, 722, 1908

Cusumano, G., Hermsen, W., Kramer, M., Kuiper, L., Löhmer, O., Massaro, E., Mineo, T., et al. 2003, A\&A, 410, L9

Edmonds, P. D., Grindlay, J. E., Cohn, H., \& Lugger, P. 2001, ApJ, 547, 829

Ergma, E., \& Sarna, M. J. 2000, A\&A, 363, 657

Foster, R. S., Backer, D. C., Taylor, J. H., \& Goss, W. M. 1988, ApJ, 326, L13

Foster, R. S., Fairhead, L., \& Backer, D. C. 1991, The Astrophysical Journal, 378,687

Fruchter, A. S., \& Goss, W. M. 2000, ApJ, 536, 865

Gil, J., \& Melikidze, G. 2004, in Young Neutron Stars and Their Environments, Vol. 218, 321

Ginzburg, V. L. 1985, Physics and astrophysics: A selection of key problems, ed. Ginzburg, V. L.

Green, D. A. 2009, Bulletin of the Astronomical Society of India, 37, 45

Hankins, T. H., \& Eilek, J. A. 2007, ApJ, 670, 693

Hankins, T. H., Kern, J. S., Weatherall, J. C., \& Eilek, J. A. 2003, Nature, 141

Harding, A. K., Stern, J. V., Dyks, J., \& Frackowiak, M. 2008, ApJ, 680, 1378

Hassall, T. E., Stappers, B. W., Hessels, J. W. T., Kramer, M., Alexov, A., Anderson, K., Coenen, T., Karastergiou, A., Keane, E. F., Kondratiev, V. I., Lazaridis, K., van Leeuwen, J., Noutsos, A., Serylak, M., Sobey, C., Verbiest, J. P. W., Weltevrede, P., Zagkouris, K., Fender, R., Wijers, R. A. M. J., Bahren, L., Bell, M. E., Broderick, J. W., Corbel, S., Daw, E. J., Dhillon, V. S., Eisloffel, J., Falcke, H., Griessmeier, J.-M., Jonker, P., Law, C., Markoff, S., Miller-Jones, J. C. A., Osten, R., Rol, E., Scaife, A. M. M., 
Scheers, B., Schellart, P., Spreeuw, H., Swinbank, J., ter Veen, S., Wise, M. W., Wijnands, R., Wucknitz, O., Zarka, P., Asgekar, A., Bell, M. R., Bentum, M. J., Bernardi, G., Best, P., Bonafede, A., Boonstra, A. J., Brentjens, M., Brouw, W. N., Bruggen, M., Butcher, H. R., Ciardi, B., Garrett, M. A., Gerbers, M., Gunst, A. W., van Haarlem, M. P., Heald, G., Hoeft, M., Holties, H., de Jong, A., Koopmans, L. V. E., Kuniyoshi, M., Kuper, G., Loose, G. M., Maat, P., Masters, J., McKean, J. P., Meulman, H., Mevius, M., Munk, H., Noordam, J. E., Orru, E., Paas, H., Pandey-Pommier, M., Pandey, V. N., Pizzo, R., Polatidis, A., Reich, W., Rottgering, H., Sluman, J., Steinmetz, M., Sterks, C. G. M., Tagger, M., Tang, Y., Tasse, C., Vermeulen, R., van Weeren, R. J., Wijnholds, S. J., \& Yatawatta, S. 2012, ArXiv e-prints

Herfindal, J. L., \& Rankin, J. M. 2009, MNRAS, 393, 1391

Hobbs, G. B., Edwards, R. T., \& Manchester, R. N. 2006, MNRAS, 369, 655

Hotan, A. W., van Straten, W., \& Manchester, R. N. 2004, PASA, 21, 302

Jessner, A., Popov, M. V., Kondratiev, V. I., Kovalev, Y. Y., Graham, D., Zensus, A., Soglasnov, et al. 2010, A\&A, 524, A60

Jessner, A., Słowikowska, A., Klein, B., Lesch, H., Jaroschek, et al. 2005, Advances in Space Research, 35, 1166

Johnston, S., \& Romani, R. W. 2002, MNRAS, 332, 109

Karuppusamy, R., Stappers, B. W., \& Serylak, M. 2011, A\&A, 525, A55+

Keane, E. F., Kramer, M., Lyne, A. G., Stappers, B. W., \& McLaughlin, M. A. 2011, MNRAS, 838

Kinkhabwala, A., \& Thorsett, S. E. 2000, ApJ, 535, 365

Knight, H. S. 2006, Chinese Journal of Astronomy and Astrophysics Supplement, 6, 020000 
Knight, H. S., Bailes, M., Manchester, R. N., Ord, S. M., \& Jacoby, B. A. 2006, ApJ, 640, 941

Kramer, M., Lyne, A. G., O'Brien, J. T., Jordan, C. A., \& Lorimer, D. R. 2006, Science, 312, 549

Kuzmin, A. D. 2006, Chinese Journal of Astronomy and Astrophysics Supplement, 6, 020000

—. 2007, Ap\&SS, 308, 563

Kuzmin, A. D., Kondrat'ev, V. I., Kostyuk, S. V., Losovsky, B. Y., Popov, M. V., Soglasnov, V. A., D’Amico, N., \& Montebugnoli, S. 2002, AstL, 28, 251, transl. from: PAZh, 2002, 28, 292

Lange, C., Kramer, M., Wielebinski, R., \& Jessner, A. 1998, A\&A, 332, 111

Lawson, K. D., Mayer, C. J., Osborne, J. L., \& Parkinson, M. L. 1987, MNRAS, 225,307

Lazaridis, K., Verbiest, J. P. W., Tauris, T. M., Stappers, B. W., Kramer, M., Wex, N., Jessner, A., Cognard, I., Desvignes, G., Janssen, G. H., Purver, M. B., Theureau, G., Bassa, C. G., \& Smits, R. 2011, MNRAS, 414, 3134

Lorimer, D. R., \& Kramer, M. 2004, Handbook of Pulsar Astronomy, ed. R. Ellis, J. Huchra, S. Kahn, G. Rieke, \& P. B. Stetson

Lundgren, S., Cordes, J., Ulmer, M., Matz, S., Lomatch, S., Foster, R., \& Hankins, T. 1995, ApJ, 453, 433

Lundgren, S. C. 1994, PhD thesis, Cornell Univ., Ithaca, NY

Lyne, A., Hobbs, G., Kramer, M., Stairs, I., \& Stappers, B. 2010, Science, 329, 408

Lyne, A., Manchester, R., D’Amico, N., Staveley-Smith, L., Johnston, S., Lim, J., Fruchter, A., Goss, W., \& Frail, D. 1990, Nature, 347, 650 
Lyne, A. G., Pritchard, R. S., \& Graham-Smith, F. 1993, MNRAS, 265, 1003

Lyutikov, M. 2007, MNRAS, 381, 1190

Lyutikov, M., Blandford, R. D., \& Machabeli, G. 1999, MNRAS, 305, 338

Machabeli, G. Z., \& Usov, V. V. 1979, Soviet Astronomy Letters, 5, 238

Malofeev, V. M., Smirnova, T. V., Soin, A. G., \& Shapovalova, N. V. 1995, Astronomy Letters, 21, 619

McLaughlin, M. A., \& Cordes, J. M. 2003, ApJ, 596, 982

McLaughlin, M. A., Lyne, A. G., Lorimer, D. R., Kramer, M., Faulkner, A. J., Manchester, R. N., Cordes, J. M., Camilo, F., Possenti, A., Stairs, I. H., Hobbs, G., D'Amico, N., Burgay, M., \& O’Brien, J. T. 2006, Nature, 439, 817

Moffett, D. A. 1997, PhD thesis, New Mexico Inst. Mining and Technology

Moffett, D. A., \& Hankins, T. H. 1996, ApJ, 468, 779

Nice, D. J., Arzoumanian, Z., \& Thorsett, S. E. 2000, in Astronomical Society of the Pacific Conference Series, Vol. 202, IAU Colloq. 177: Pulsar Astronomy - 2000 and Beyond, ed. M. Kramer, N. Wex, \& R. Wielebinski, 67

Nice, D. J., \& Thorsett, S. E. 1992, ApJ, 397, 249

Ord, S. M., van Straten, W., Hotan, A. W., \& Bailes, M. 2004, MNRAS, 352, 804

Patt, B. L., Ulmer, M. P., Zhang, W., Cordes, J. M., \& Arzoumanian, Z. 1999, ApJ, 522, 440

Pellizzoni, A., Pilia, M., Possenti, A., Chen, A., Giuliani, A., Trois, A., Caraveo, P., Del Monte, E., Fornari, F., Fuschino, F., Mereghetti, S., Tavani, M., Argan, A., Burgay, M., Cognard, I., Corongiu, A., Costa, E., D’Amico, N., 
De Luca, A., Esposito, P., Evangelista, Y., Feroci, M., Johnston, S., Kramer, M., Longo, F., Marisaldi, M., Theureau, G., Weltevrede, P., Barbiellini, G., Boffelli, F., Bulgarelli, A., Cattaneo, P. W., Cocco, V., D'Ammando, F., DeParis, G., Di Cocco, G., Donnarumma, I., Fiorini, M., Froysland, T., Galli, M., Gianotti, F., Labanti, C., Lapshov, I., Lazzarotto, F., Lipari, P., Mineo, T., Morselli, A., Pacciani, L., Perotti, F., Piano, G., Picozza, P., Prest, M., Pucella, G., Rapisarda, M., Rappoldi, A., Sabatini, S., Soffitta, P., Trifoglio, M., Vallazza, E., Vercellone, S., Vittorini, V., Zambra, A., Zanello, D., Pittori, C., Verrecchia, F., Preger, B., Santolamazza, P., Giommi, P., Salotti, L., \& Bignami, G. F. 2009, ApJ, 695, L115

Petrova, S. A. 2006, Chinese Journal of Astronomy and Astrophysics Supplement, 6, 020000

Phinney, E. S. 1992, Royal Society of London Philosophical Transactions Series A, 341, 39

Popov, M. V., Bartel, N., Cannon, W. H., Novikov, A. Y., Kondratiev, V. I., \& Altunin, V. I. 2002, A\&A, 396, 171

Popov, M. V., Kuzmin, A. D., Ulyanov, O. V., Deshpande, A. A., Ershov, A. A., et al. 2006, ARep, 50, 562, transl. from: AZh, 2006, 83, 630

Popov, M. V., \& Stappers, B. 2007, A\&A, 470, 1003

Radhakrishnan, V., \& Cooke, D. J. 1969, Astrophys. Lett., 3, 225

Ramanamurthy, P. V., \& Thompson, D. J. 1998, ApJ, 496, 863

Rickett, B. J. 1977, ARA\&A, 15, 479

Rickett, B. J., \& Lyne, A. G. 1990, MNRAS, 244, 68

Romani, R. W., \& Johnston, S. 2001, The Astrophysical Journal, 557, L93

Rots, A. H., Jahoda, K., \& Lyne, A. G. 2004, ApJ, 605, L129 
Sallmen, S., Backer, D. C., Hankins, T. H., Moffett, D., \& Lundgren, S. 1999, 517,460

Shearer, A., Stappers, B., O'Connor, P., Golden, A., Strom, R., Redfern, M., \& Ryan, O. 2003, Science, 301, 493

Smirnova, T. V., \& Shishov, V. I. 2008, Astronomy Reports, 52, 736

Soglasnov, V. A., Popov, M. V., Bartel, N., Cannon, W., Novikov, A. Y., Kondratiev, V. I., \& Altunin, V. I. 2004, ApJ, 616, 439

Staelin, D. H., \& Reifenstein III, E. C. 1968, Science, 162, 1481

Stairs, I. H., Thorsett, S. E., \& Camilo, F. 1999, ApJS, 123, 627

Stappers, B. W., Hessels, J. W. T., Alexov, A., Anderson, K., Coenen, T., Hassall, T., Karastergiou, A., Kondratiev, V. I., Kramer, M., van Leeuwen, J., Mol, J. D., Noutsos, A., Romein, J. W., Weltevrede, P., Fender, R., Wijers, R. A. M. J., Bähren, L., Bell, M. E., Broderick, J., Daw, E. J., Dhillon, V. S., Eislöffel, J., Falcke, H., Griessmeier, J., Law, C., Markoff, S., Miller-Jones, J. C. A., Scheers, B., Spreeuw, H., Swinbank, J., Ter Veen, S., Wise, M. W., Wucknitz, O., Zarka, P., Anderson, J., Asgekar, A., Avruch, I. M., Beck, R., Bennema, P., Bentum, M. J., Best, P., Bregman, J., Brentjens, M., van de Brink, R. H., Broekema, P. C., Brouw, W. N., Brüggen, M., de Bruyn, A. G., Butcher, H. R., Ciardi, B., Conway, J., Dettmar, R.-J., van Duin, A., van Enst, J., Garrett, M., Gerbers, M., Grit, T., Gunst, A., van Haarlem, M. P., Hamaker, J. P., Heald, G., Hoeft, M., Holties, H., Horneffer, A., Koopmans, L. V. E., Kuper, G., Loose, M., Maat, P., McKay-Bukowski, D., McKean, J. P., Miley, G., Morganti, R., Nijboer, R., Noordam, J. E., Norden, M., Olofsson, H., Pandey-Pommier, M., Polatidis, A., Reich, W., Röttgering, H., Schoenmakers, A., Sluman, J., Smirnov, O., Steinmetz, M., Sterks, C. G. M., Tagger, M., Tang, Y., Vermeulen, R., Vermaas, N., Vogt, C., de Vos, M., Wijnholds, S. J., Yatawatta, S., \& Zensus, A. 2011, A\&A, 530, A80+ 
Thompson, C., Blandford, R., Evans, C., \& Phinney, E. 1994, The Astrophysical Journal, 422, 304

Ulyanov, O. M., Deshpande, A. A., Zakharenko, V. V., Asgekar, A., \& Shankar, U. 2007, Radiofizika and Radioastronomia, 12, 5

Ul'yanov, O. M., \& Zakharenko, V. V. 2012, Astronomy Reports, 56, 417

Ulyanov, O. M., Zakharenko, V. V., Konovalenko, A. A., Lecacheux, A., Rosolen, C., \& Rucker, H. O. 2006, Radiofizika and Radioastronomia, 11, 113

van Straten, W. 2004, ApJS, 152, 129

van Straten, W., \& Bailes, M. 2011, PASA, 28, 1

van Straten, W., Manchester, R. N., Johnston, S., \& Reynolds, J. E. 2010, PASA, 27, 104

Verbiest, J., Bailes, M., Coles, W., Hobbs, G., van Straten, W., Champion, D., Jenet, F., Manchester, R., Bhat, N., Sarkissian, J., Yardley, D., BurkeSpolaor, S., Hotan, A., \& You, X. 2009, Monthly Notices of the Royal Astronomical Society, 400, 951

Vivekanand, M. 2001, A\&A, 373, 236

Weatherall, J. C. 1998, ApJ, 506, 341

Weltevrede, P., Wright, G. A. E., Stappers, B. W., \& Rankin, J. M. 2006, A\&A, 458,269

Yan, W. M., Manchester, R. N., Hobbs, G., van Straten, W., Reynolds, J. E., Wang, N., Bailes, M., Bhat, N. D. R., Burke-Spolaor, S., Champion, D. J., Chaudhary, A., Coles, W. A., Hotan, A. W., Khoo, J., Oslowski, S., Sarkissian, J. M., \& Yardley, D. R. B. 2011a, 335, 485 
Yan, W. M., Manchester, R. N., van Straten, W., Reynolds, J. E., Hobbs, G., Wang, N., Bailes, M., Bhat, N. D. R., Burke-Spolaor, S., Champion, D. J., Coles, W. A., Hotan, A. W., Khoo, J., Oslowski, S., Sarkissian, J. M., Verbiest, J. P. W., \& Yardley, D. R. B. 2011b, MNRAS, 414, 2087 